\title{
Resonance frequency and radiative Q-factor of plasmonic and dielectric modes of small objects
}

\author{
Carlo Forestiere $\odot,{ }^{*}$ Giovanni Miano, and Guglielmo Rubinacci $\odot$ \\ Department of Electrical Engineering and Information Technology, Università degli Studi di Napoli Federico II, \\ via Claudio 21, Napoli, 80125, Italy
}

(Received 12 May 2020; revised 21 August 2020; accepted 11 September 2020; published 3 November 2020; corrected 9 July 2021)

\begin{abstract}
The electromagnetic scattering resonances and modes of a nonmagnetic object much smaller than the incident wavelength in vacuum can be either described by the electroquasistatic approximation of the Maxwell's equations if its permittivity is negative or by the magnetoquasistatic approximation if its permittivity is positive and sufficiently high. Nevertheless, these two approximations fail to correctly account for the frequency shift and the radiative broadening of the resonances when the size of the object becomes comparable to the wavelength of operation. In this paper, the radiation corrections to the electroquasistatic and magnetoquasistatic resonances and modes of arbitrarily shaped objects are derived, which only depend on the quasistatic current modes. Then, closed-form expressions of the frequency shift and the radiative Q-factor of both plasmonic and dielectric modes of small objects are introduced, where the dependencies on the material and the size of the object are factorized. In particular, it is shown that the radiative Q-factor explicitly depends on the multipolar components of the quasistatic mode and its corrections.
\end{abstract}

DOI: 10.1103/PhysRevResearch.2.043176

\section{INTRODUCTION}

There exist two mechanisms through which a nonmagnetic homogeneous object, assumed small compared to the incident wavelength in vacuum, may resonate.

The first resonance mechanism occurs in small metal nanoparticles with negative permittivity, and it arises from the interplay between the energy stored in the electric field and the kinetic energy of the free electrons of the metal. When the object is very small compared to the wavelength in vacuum, these resonances are well described by the electroquasistatic approximation of the Maxwell's equations [1-5] and associated to the negative values of permittivity in correspondence of which source-free solutions exist. However, it is known that as the size of the object becomes comparable to the incident wavelength, this approximation is unable to describe the radiative shift and broadening of these resonances.

The second resonance mechanism occurs in small objects of high and positive permittivity, and it arises from the interplay between the polarization energy stored in the dielectric and the energy stored in the magnetic field. Manifestation of this kind of resonance can be found at microwave [6-9] and optical [10-14] frequencies. When the object is very small compared to the free-space wavelength, and the permittivity very high, these resonances are well described by the magnetoquasistatic approximation of the Maxwell's equations [15], where the normal component of the displacement

\footnotetext{
*carlo.forestiere@unina.it

Published by the American Physical Society under the terms of the Creative Commons Attribution 4.0 International license. Further distribution of this work must maintain attribution to the author(s) and the published article's title, journal citation, and DOI.
}

current density field vanishes on the surface of the particle $[15,16]$. In particular, these resonances are associated with the eigenvalues of the magnetostatic integral operator expressing the vector potential in terms of the displacement current density [15]. Unfortunately, when the permittivity of the dielectric material is moderately high, as happens, for instance. in the visible spectral range [10-14], the size of the object has to be comparable to the incident wavelength to trigger a resonant response. In this scenario, the magnetoquasistatic approximation is unable to describe the frequency shift and the broadening of these resonances.

In light of these observations, to describe the electromagnetic resonances of objects of size comparable to the incident wavelength, one may be tempted to abandon the quasistatic approximation altogether and then turn to the full Maxwell's equations, using one of several possible spectral approaches, including the quasinormal modes [17,18], the characteristic modes [19,20], or the material-independent modes [21,22]. There are certainly some advantages in doing so, including the fact that the full-wave formulations would enable, given unlimited computational resources, the treatment of objects of any size. In fact, scattering resonances have been already investigated by considering full-wave eigenvalue problems based on volume (e.g., Refs. [23-25]), surface (e.g., Refs. [26-30]), and line integral formulations of the Maxwell's equations [31]; differential formulations (e.g., Refs. [32,33]); and Mie theory (e.g., Refs. [17,22,34,35]). For a recent review, see Ref. [18]. However, the resulting resonance frequencies and Q-factors depend on the morphology, material, and size of the object. A change of any of these parameters would require an entirely new calculation. These dependencies are buried below the computational layer and cannot be factorized.

Closed-form expressions of the Q-factor and the frequency shift of both plasmonic and dielectric modes, where the 
dependencies on the material and size of the object are factorized, are highly desirable. They would enable the classification of the resonances and facilitate their engineering [36], including the coupling with emitters [37-40], because they could be used as a target for the design [41,42]. Moreover, in many applications, the size of metal or dielectric objects does not exceed the free-space wavelength of operation [13]. These are powerful incentives to pursue the extension of the two quasistatic scattering limits to include radiation effects.

In the literature, there already exist closed-form expressions for the resonance frequency shift and Q-factors in few scenarios.

For electrically small antennas, physical limitations on the Q-factor have been the subject of numerous papers [43-48], starting from the classical works of Wheeler [49] and Chu [50].

For plasmonic resonators, Mayergoyz et al. derived the second-order correction to electroquasistatic eigenvalues [51], starting from Maxwell's equations in differential form. Wang and Shen derived the expression of the Q-factor of a plasmonic mode, when the nonradiative losses are dominant [4]. The derivation of a general expression for the Q-factor of plasmonic modes when radiative losses are dominant [52] remains unexplored.

For high-index dielectric resonators, Van Bladel introduced closed-form expressions for the Q-factor of the magnetic dipole mode and provided the Q-factor of specific higher order modes of a rotationally symmetric object [16], considering an asymptotic expansion of the Maxwell's equation in differential form in terms of the inverse of the index of refraction. Following Van Bladel's work, De Smedt derived the frequency shift and Q-factor of a rotationally symmetric ring resonator [53]. General expressions for both the frequency shift and radiative factor have not been derived yet.

In this paper, the radiation corrections for both the electroquasistatic and magnetoquasistatic resonances and resonant modes of arbitrarily shaped nonmagnetic homogeneous and isotropic objects are introduced, using an integral formulation of the Maxwell's equations and treating the size parameter of the object as a small parameter.

Then, closed-form expressions of the frequency shift and the radiative Q-factor of both plasmonic and dielectric modes of small objects are introduced, where the dependencies on the material and the size of the object are factorized. Specifically, the relative resonance frequency shift of any mode (with respect to the quasistatic resonance position) is a quadratic function of the size parameter at the quasistatic resonance, whose prefactor depends on the ratio between the secondorder eigenvalue correction and the quasistatic eigenvalue. Furthermore, the radiative Q-factor is an inverse power function of the size parameter whose exponent is the order $n_{i}$ of the first nonvanishing imaginary eigenvalue correction, while the prefactor is the ratio between the quasistatic eigenvalue and the $n_{i}$ th-order imaginary correction, which explicitly depends on the multipolar components of the quasistatic mode.

This paper is organized as follows. First, the scattering resonances and modes in the quasistatic regimes are briefly summarized in Sec. II. Then, in Sec. III, the fullwave scattering problem is formulated and an eigenvalue problem governing the scattering resonances and modes is introduced. This eigenvalue problem is solved perturbatively in Secs. IV and V, starting from the electroquasistatic and magnetoquasistatic limits, treating the size parameter as a small parameter. Collecting same-order terms, closed-form radiation corrections are found. In Sec. VI, the frequency shift and the Q-factor are obtained as a function of these radiation corrections. In Sec. VII, the catalogs of plasmonic and photonic resonances are introduced. They constitute a synthetic classification of the modes of a homogeneous nonmagnetic objects, and depend only on its morphology but not on its size, material, and frequency of operations. This classification may help the description of the elementary building blocks of the nanocircuitry envisioned by Engheta et al. in Ref. [54]. Eventually, the introduced formalism is validated by investigating the resonance frequency and Q-factors in the scattering response of metal and dielectric objects of size comparable to the incident wavelength.

\section{MODES AND RESONANCES IN THE QUASISTATIC REGIME}

A homogeneous, isotropic, nonmagnetic, linear material occupies a volume $\Omega$, of characteristic linear dimension $l_{c}$, bounded by a closed surface $\partial \Omega$ with an outward-pointing normal $\hat{\mathbf{n}}$. The material has relative permittivity $\varepsilon_{R}$, and it is surrounded by vacuum. There exist two mechanisms through this object may resonate in the quasistatic regime [15].

\section{A. Electroquasistatic modes and resonances}

The first resonance mechanism is the electroquasistatic resonance, occurring in metals (more in general, in objects whose dielectric permittivity has a negative real part) where the induced electric charge plays a central role. These resonances are associated with the eigenvalues $\chi_{h}^{\|}$of the electrostatic integral operator $\mathscr{L}_{e}$ that gives the electrostatic field as a function of the surface charge density [55]:

$$
\chi_{h}^{\|} \mathscr{L}_{e}\left\{\mathbf{j}_{h}^{\|}\right\}=\mathbf{j}_{h}^{\|},
$$

where the expression of $\mathscr{L}_{e}$ is

$$
\mathscr{L}_{e}\left\{\mathbf{j}_{h}^{\|}\right\}=-\tilde{\nabla} \oint_{\partial \tilde{\Omega}} g_{0}\left(\tilde{\mathbf{r}}-\tilde{\mathbf{r}}^{\prime}\right) \mathbf{j}_{h}^{\|}\left(\tilde{\mathbf{r}}^{\prime}\right) \cdot \hat{\mathbf{n}}\left(\tilde{\mathbf{r}}^{\prime}\right) d \tilde{S}^{\prime},
$$

$g_{0}\left(\tilde{\mathbf{r}}-\tilde{\mathbf{r}}^{\prime}\right)=\frac{1}{4 \pi\left|\tilde{\mathbf{r}}-\tilde{\mathbf{r}}^{\prime}\right|}$ is the static Green's function in vacuum. In Eqs. (1) and (2), the spatial coordinates have been normalized by $l_{c}$, i.e., $\tilde{\mathbf{r}}=\mathbf{r} / l_{c}, \tilde{\Omega}$ is the corresponding scaled domain, $\partial \tilde{\Omega}$ is the boundary of $\tilde{\Omega}$, and $\tilde{\nabla}$ is the scaled gradient operator.

The quasistatic oscillations, represented by the electroquasistatic (EQS) current modes $\mathbf{j}_{h}^{\|}$, arise from the interplay between the energy stored in the electric field and the kinetic energy of the free electrons in the metal. The spectrum of the operator $\mathscr{L}_{e}$ is discrete $[51,55]$. Each EQS mode $\mathbf{j}_{h}^{\|}$is characterized by a real and negative eigenvalue $\chi_{h}^{\|}$, which is size independent [51]. The modes $\left\{\mathbf{j}_{h}^{\|}\right\}_{h \in \mathbb{N}}$ are longitudinal vector fields: They are both curl-free and divergence-free (div-free) within the object but have nonvanishing normal component to the object surface [51,55]. This normal component is related to the induced surface charge density on $\partial \tilde{\Omega}$, and satisfies the 
charge-neutrality condition, i.e.,

$$
\oint_{\partial \tilde{\Omega}} \mathbf{j}_{h}^{\|}\left(\tilde{\mathbf{r}}^{\prime}\right) \cdot \tilde{\mathbf{n}}\left(\tilde{\mathbf{r}}^{\prime}\right) d \tilde{S}^{\prime}=0 .
$$

Moreover, the EQS modes are orthonormal,

$$
\left\langle\mathbf{j}_{h}^{\|}, \mathbf{j}_{k}^{\|}\right\rangle_{\tilde{\Omega}}=\delta_{h, k},
$$

accordingly to the scalar product

$$
\langle\mathbf{f}, \mathbf{g}\rangle_{\tilde{\Omega}}=\int_{\tilde{\Omega}} \mathbf{f}^{*}(\tilde{\mathbf{r}}) \cdot \mathbf{g}(\tilde{\mathbf{r}}) d \tilde{V} .
$$

Under the normalization (4), the electrostatic energy of the $h$ th EQS current mode is

$$
\mathscr{W}_{e}\left\{\mathbf{j}_{h}^{\|}\right\}=\frac{1}{2 \varepsilon_{0}} \frac{1}{\left(-\chi_{h}^{\|}\right)} .
$$

The electric dipole moment $\mathbf{P}_{\mathrm{E}, h}^{\|}$of the EQS mode $\mathbf{j}_{h}^{\|}$is defined accordingly to Eq. (A1) of Appendix A. If the mode $\mathbf{j}_{h}^{\|}$exhibits a vanishing electric dipole moment, i.e.,

$$
\left\|\mathbf{P}_{\mathrm{E}, h}^{\|}\right\|=0,
$$

it is called dark; otherwise it is bright.

\section{B. Magnetoquasistatic modes and and resonances}

The second resonance mechanism is the magnetoquasistatic resonance, occurring in dielectric objects with high and positive permittivity, where the displacement current density field plays a central role. These resonances are associated with the eigenvalues $\kappa_{h}^{\perp}$ of the magnetostatic integral operator $\mathscr{L}_{m}$ that gives the vector potential as a function of the current density [15],

$$
\kappa_{h}^{\perp} \mathscr{L}_{m}\left\{\mathbf{j}_{h}^{\perp}\right\}=\mathbf{j}_{h}^{\perp},
$$

with

$$
\left.\mathbf{j}_{h}^{\perp}(\tilde{\mathbf{r}}) \cdot \hat{\mathbf{n}}(\tilde{\mathbf{r}})\right|_{\partial \tilde{\Omega}}=\mathbf{0} \quad \forall \tilde{\mathbf{r}} \in \partial \tilde{\Omega},
$$

where $\mathscr{L}_{m}$ is

$$
\mathscr{L}_{m}\left\{\mathbf{j}_{h}^{\perp}\right\}(\tilde{\mathbf{r}})=\int_{\tilde{\Omega}} g_{0}\left(\tilde{\mathbf{r}}-\tilde{\mathbf{r}}^{\prime}\right) \mathbf{j}_{h}^{\perp}\left(\tilde{\mathbf{r}}^{\prime}\right) d \tilde{V}^{\prime} .
$$

Equation (8) holds in the weak form in the functional space equipped with the inner product (5), and constituted by the transverse vector fields which are div-free within $\tilde{\Omega}$ and having zero normal component to $\partial \tilde{\Omega}$.

The quasistatic oscillations represented by the magnetoquasistatic (MQS) current density modes $\mathbf{j}_{h}^{\perp}$ arise from the interplay between the polarization energy stored in the dielectric and the energy stored in the magnetic field [15]. The spectrum of the magnetoquasistatic operator (8) is discrete, too [15]. The MQS current mode $\mathbf{j}_{h}^{\perp}$ is characterized by a real and positive eigenvalue $\kappa_{h}^{\perp}$, which is size independent. The current modes $\left\{\mathbf{j}_{h}^{\perp}\right\}_{h \in \mathbb{N}}$ are transverse modes: They have a nonzero curl within the object, but are divergence-free and have a vanishing normal component on the object surface [15]. Each current mode $\mathbf{j}_{h}^{\perp}$ has zero electric dipole moment, namely,

$$
\int_{\tilde{\Omega}} \mathbf{j}_{h}^{\perp}\left(\tilde{\mathbf{r}}^{\prime}\right) d \tilde{V}=0
$$

Furthermore, the MQS current density modes are orthonormal:

$$
\left\langle\mathbf{j}_{h}^{\perp} \mid \mathbf{j}_{k}^{\perp}\right\rangle=\delta_{h, k}
$$

Under this normalization, the magnetostatic energy of the $h$ th electroquasistatic current mode is

$$
\mathscr{W}_{m}\left\{\mathbf{j}_{h}^{\perp}\right\}=\frac{\mu_{0}}{2} \frac{1}{\kappa_{h}^{\perp}} .
$$

The current mode $\mathbf{j}_{h}^{\perp}$ generates a vector potential

$$
\mathbf{A}\left\{\mathbf{j}_{h}^{\perp}\right\}(\tilde{\mathbf{r}})=\frac{\mu_{0}}{4 \pi} \int_{\tilde{\Omega}} g_{0}\left(\tilde{\mathbf{r}}-\tilde{\mathbf{r}}^{\prime}\right) \mathbf{j}_{h}^{\perp}\left(\tilde{\mathbf{r}}^{\prime}\right) d \tilde{V}^{\prime} .
$$

Among the MQS current modes, there exists a subset of modes generating a vector potential $\mathbf{A}\left\{\mathbf{j}_{h}^{\perp}\right\}$ with zero normal component to $\partial \tilde{\Omega}$, i.e.,

$$
\hat{\mathbf{n}}(\tilde{\mathbf{r}}) \cdot \mathbf{A}\left\{\mathbf{j}_{h}^{\perp}\right\}(\tilde{\mathbf{r}})=0, \quad \forall \tilde{\mathbf{r}} \in \partial \tilde{\Omega} ;
$$

namely $\mathbf{A}\left\{\mathbf{j}_{h}^{\perp}\right\}(\tilde{\mathbf{r}})$ is a transverse field. In this paper, a MQS mode belonging to this subset is called $\mathbb{A}^{\perp}$ mode. The $\mathbb{A}^{\perp}$ modes are also solution of the problem (8) in a strong form (in the space of square integrable vector fields).

The longitudinal set of EQS current modes $\left\{\mathbf{j}_{h}^{\|}\right\}_{h \in \mathbb{N}}$ and the transverse set of MQS $\left\{\mathbf{j}_{h}^{\perp}\right\}_{h \in \mathbb{N}}$ modes are orthogonal accordingly to the scalar product (5), and together are a complete basis of the vector space of square integrable divergence-free vector fields in $\Omega$.

\section{ELECTROMAGNETIC MODES AND RESONANCES}

The full-wave scattering problem can be formulated by considering as unknown the current density field $\mathbf{J}$ induced in the object. This current density particularizes into conduction current in metals at frequencies below interband transitions, polarization current in dielectrics, sum of conduction and polarization currents in metals in the frequency ranges where interband transitions occur. The object is illuminated by a time harmonic electromagnetic field $\operatorname{Re}\left\{\mathbf{E}_{\text {inc }}(\mathbf{r}) e^{i \omega t}\right\}$. In the frequency domain, the field $\mathbf{J}(\mathbf{r})$ is related to the electric field $\mathbf{E}(\mathbf{r})$ by $\mathbf{J}(\mathbf{r})=i \omega \varepsilon_{0} \chi(\omega) \mathbf{E}(\mathbf{r})$ where $\chi(\omega)=\left(\varepsilon_{R}(\omega)-1\right)$ is the electric susceptibility of the object and $\varepsilon_{0}$ is the vacuum permittivity. Both the vector field $\mathbf{E}$ and $\mathbf{J}$ are divergence-free in the region $\Omega$ occupied by the object due to the homogeneity and isotropy of the material. The induced current density is solution of the full-wave volume integral equation [56-58]:

$$
\begin{aligned}
\frac{\mathbf{J}(\mathbf{r})}{i \omega \varepsilon_{0} \chi}= & -\frac{1}{i \omega \varepsilon_{0}} \nabla \oint_{\partial \Omega} g\left(\mathbf{r}-\mathbf{r}^{\prime}\right) \mathbf{J}\left(\mathbf{r}^{\prime}\right) \cdot \hat{\mathbf{n}}\left(\mathbf{r}^{\prime}\right) d S^{\prime} \\
& -i \omega \mu_{0} \int_{\Omega} g\left(\mathbf{r}-\mathbf{r}^{\prime}\right) \mathbf{J}\left(\mathbf{r}^{\prime}\right) d V^{\prime}+\mathbf{E}_{i n c}(\mathbf{r}), \\
& \forall \mathbf{r} \in \Omega,
\end{aligned}
$$

where $\mu_{0}$ is the vacuum permeability, $g\left(\mathbf{r}-\mathbf{r}^{\prime}\right)=$ $e^{-i k_{0} r} / 4 \pi\left|\mathbf{r}-\mathbf{r}^{\prime}\right|$ is the Green's function in vacuum, $k_{0}=\omega / c_{0}$, and $c_{0}=1 / \sqrt{\varepsilon_{0} \mu_{0}}$. The surface and volume integrals represent the contributions to the induced electric field of the scalar and vector potentials, respectively. Then, 
Eq. (16) is rewritten as [25]

$$
\frac{\mathbf{J}(\tilde{\mathbf{r}})}{\chi}-\mathscr{L}\{\mathbf{J}\}(\tilde{\mathbf{r}})=i \omega \varepsilon_{0} \mathbf{E}_{i n c}(\tilde{\mathbf{r}}), \quad \forall \tilde{\mathbf{r}} \in \tilde{\Omega},
$$

where the spatial coordinates are normalized as $\tilde{\mathbf{r}}=\mathbf{r} / l_{c}$,

$$
\begin{aligned}
\mathscr{L}\{\mathbf{J}\}(\tilde{\mathbf{r}})= & -\tilde{\nabla} \oint_{\partial \tilde{\Omega}} g\left(\tilde{\mathbf{r}}-\tilde{\mathbf{r}}^{\prime}, x\right) \mathbf{J}\left(\tilde{\mathbf{r}}^{\prime}\right) \cdot \hat{\mathbf{n}}\left(\tilde{\mathbf{r}}^{\prime}\right) d \tilde{S}^{\prime} \\
& +x^{2} \int_{\tilde{\Omega}} g\left(\tilde{\mathbf{r}}-\tilde{\mathbf{r}}^{\prime}, x\right) \mathbf{J}\left(\tilde{\mathbf{r}}^{\prime}\right) d \tilde{V}^{\prime},
\end{aligned}
$$

$\tilde{\Omega}$ is the scaled domain, $\partial \tilde{\Omega}$ is boundary of $\tilde{\Omega}, \tilde{\nabla}$ is the scaled gradient operator, $x$ is the size parameter $x=2 \pi l_{c} / \lambda$, $g\left(\tilde{\mathbf{r}}-\tilde{\mathbf{r}}^{\prime}, x\right)$ is the Green's function in vacuum

$$
g\left(\tilde{\mathbf{r}}-\tilde{\mathbf{r}}^{\prime}, x\right)=\frac{1}{4 \pi} \frac{e^{-i x\left|\tilde{\mathbf{r}}-\tilde{\mathbf{r}}^{\prime}\right|}}{\left|\tilde{\mathbf{r}}-\tilde{\mathbf{r}}^{\prime}\right|}=\frac{1}{4 \pi} \frac{e^{-i x \Delta \tilde{\mathbf{r}}}}{\Delta \tilde{\mathbf{r}}},
$$

and $\Delta \tilde{\mathbf{r}}=\left|\tilde{\mathbf{r}}-\tilde{\mathbf{r}}^{\prime}\right|$.

The spectral properties of the linear operator $\mathscr{L}$ play a very important role in any resonant scattering mechanism. Since $\mathscr{L}$ is compact its spectrum is countable infinite. The operator $\mathscr{L}$ is symmetric but not self-adjoint. For any value of the size parameter $x$, its eigenvalues are complex with positive imaginary part. The eigenfunctions corresponding to two different eigenvalues are not orthogonal in the usual sense: They are biorthogonal $[21,22]$.

The eigenvalue problem [25]

$$
\mathscr{L}\left\{\mathbf{j}_{h}\right\}=\frac{1}{\gamma_{h}} \mathbf{j}_{h}
$$

splits into the two eigenvalue problems (1) and (8) (see Secs. II A and II B) in the quasistatic regime $x \ll 1$ (small object). This fact was already shown for 2D objects in Ref. [30] and for 3D objects in Ref. [15]. The eigenfunctions of $\mathscr{L}$ that in the limit $x \rightarrow 0$ tend to the EQS modes are indicated with $\left\{\mathbf{u}_{h}(\tilde{\mathbf{r}})\right\}$ and the corresponding eigenvalues are indicated as $\left\{\chi_{h}\right\}$. These eigenfunctions are called plasmonic modes. Dually, the set of eigenfunctions of $\mathscr{L}$ that in the limit $x \rightarrow 0$ tend to the MQS modes are indicated with $\left\{\mathbf{v}_{h}(\tilde{\mathbf{r}})\right\}$ and the corresponding eigenvalues are indicated with $\kappa_{h} / x^{2}$. Although in the limit $x \rightarrow 0$, the eigenvalues $\kappa_{h} / x^{2}$ diverge, the quantities $\kappa_{h}$ remain constant. These eigenfunctions are called dielectric modes. Forestiere and Miano et al. in Refs. [22,34] used the adjectives plasmonic and photonic mode instead of plasmonic and dielectric mode to identify the same two sets, while in Ref. [35] the authors called them longitudinal and transverse modes. All these nomenclatures are equivalent. It was shown that this two sets of modes, even if this distinction is made in the long-wavelength regime, remain well distinguishable and have different properties even in the full-wave regime $[22,30,34]$.

The union of the two sets $\left\{\mathbf{u}_{h}(\tilde{\mathbf{r}})\right\}$ and $\left\{\mathbf{v}_{h}(\tilde{\mathbf{r}})\right\}$ is a basis for the unknown current density field in Eq. (16). Its solution is expressed as

$$
\begin{aligned}
\mathbf{J}(\tilde{\mathbf{r}})= & \chi\left[\sum_{h=1}^{\infty} \frac{\chi_{h}}{\chi_{h}-\chi}\left\langle\mathbf{u}_{h}^{*}, \mathbf{E}_{i n c}\right\rangle_{\tilde{\Omega}} \mathbf{u}_{h}(\tilde{\mathbf{r}})\right. \\
& \left.+\sum_{h=1}^{\infty} \frac{\kappa_{h}}{\kappa_{h}-\chi x^{2}}\left\langle\mathbf{v}_{h}^{*}, \mathbf{E}_{i n c}\right\rangle_{\tilde{\Omega}} \mathbf{v}_{h}(\tilde{\mathbf{r}})\right],
\end{aligned}
$$

where both the set of modes $\left\{\mathbf{u}_{h}(\tilde{\mathbf{r}})\right\}$ and $\left\{\mathbf{v}_{h}(\tilde{\mathbf{r}})\right\}$ are normalized, $\left\langle\mathbf{u}_{h}^{*}, \mathbf{u}_{h}\right\rangle=1$, and $\left\langle\mathbf{v}_{h}^{*}, \mathbf{v}_{h}\right\rangle=1$ for any $h$. This expansion is very useful because it separates the dependence on the material from the dependence on the geometry $[21,22,25,30,31]$ and has been used in different contexts $[34,59,60]$.

In the next two sections, we develop a perturbation theory to evaluate the plasmonic and dielectric resonances and resonant modes of an object with arbitrary shape and size parameter $x \lesssim 1$, by starting from the corresponding modes in the quasistatic regime.

\section{PLASMONIC MODES AND RESONANCES}

To evaluate the plasmonic resonances of small particles, it is convenient to recast the eigenvalue problem (20) as

$$
\begin{aligned}
& -\mathbf{u}_{h}(\tilde{\mathbf{r}})-\chi_{h} \tilde{\nabla} \oint_{\partial \tilde{\Omega}} g\left(\tilde{\mathbf{r}}-\tilde{\mathbf{r}}^{\prime}, x\right) \mathbf{u}_{h}\left(\tilde{\mathbf{r}}^{\prime}\right) \cdot \hat{\mathbf{n}}\left(\tilde{\mathbf{r}}^{\prime}\right) d \tilde{S}^{\prime} \\
& \quad+\chi_{h} x^{2} \int_{\tilde{\Omega}} g\left(\tilde{\mathbf{r}}-\tilde{\mathbf{r}}^{\prime}, x\right) \mathbf{u}_{h}\left(\tilde{\mathbf{r}}^{\prime}\right) d \tilde{V}^{\prime}=\mathbf{0} \quad \forall \tilde{\mathbf{r}} \in \tilde{\Omega} .
\end{aligned}
$$

When the free-space wavelength $\lambda=2 \pi c_{0} / \omega$ is large in comparison with the characteristic dimension $l_{c}$, the size parameter $x$ can be treated as a small parameter, and the Green's function $g\left(\tilde{\mathbf{r}}-\tilde{\mathbf{r}}^{\prime}, x\right)$, the current mode $\mathbf{u}_{h}$, and the eigenvalue $\chi_{h}$ can all be expanded in terms of $x$ in the neighborhood of the EQS resonance with eigenvalue $\chi_{h}^{\|}$and mode $\mathbf{j}_{h}^{\|}$:

$$
\begin{aligned}
\chi_{h} & =\chi_{h}^{\|}+\chi_{h}^{(1)} x+\chi_{h}^{(2)} x^{2}+\chi_{h}^{(3)} x^{3}+\cdots \\
& =\sum_{k=0}^{\infty} \chi_{h}^{(k)} x^{k}, \\
\mathbf{u}_{h} & =\mathbf{j}_{h}^{\|}+\mathbf{u}_{h}^{(1)} x+\mathbf{u}_{h}^{(2)} x^{2}+\mathbf{u}_{h}^{(3)} x^{3}+\ldots \\
& =\sum_{k=0}^{\infty} \mathbf{u}_{h}^{(k)} x^{k}, \\
g\left(\tilde{\mathbf{r}}-\tilde{\mathbf{r}}^{\prime}, x\right) & =\frac{1}{4 \pi}\left(\Delta \tilde{\mathbf{r}}^{-1}-i x-\frac{\Delta \tilde{\mathbf{r}}}{2 !} x^{2}+i \frac{\Delta \tilde{\mathbf{r}}^{2}}{3 !} x^{3}+\cdots\right) \\
& =\frac{1}{4 \pi} \sum_{k=0}^{\infty}(-i)^{k} \frac{\Delta \tilde{\mathbf{r}}^{k-1}}{k !} x^{k} .
\end{aligned}
$$

By using Eqs. (23), (24), and (25), Eq. (22) becomes

$$
\begin{aligned}
& -4 \pi \sum_{k=0}^{\infty} \mathbf{u}_{h}^{(k)} x^{k}-\left(\sum_{k=0}^{\infty} \chi_{h}^{(k)} x^{k}\right) \tilde{\nabla} \oint_{\partial \tilde{\Omega}}\left(\sum_{k=0}^{\infty}(-i)^{k} \frac{\Delta \tilde{\mathbf{r}}^{k-1}}{k !} x^{k}\right)\left(\sum_{k=0}^{\infty} u_{n, h}^{(k)} x^{k}\right) d \tilde{S}^{\prime} \\
& +\left(\sum_{k=0}^{\infty} \chi_{h}^{(k)} x^{k}\right) \int_{\tilde{\Omega}}\left(\sum_{k=0}^{\infty}(-i)^{k} \frac{\Delta \tilde{\mathbf{r}}^{k-1}}{k !} x^{k}\right)\left(\sum_{k=0}^{\infty} \mathbf{u}_{h}^{(k)} x^{k+2}\right) d \tilde{V}^{\prime}=0, \quad \forall \mathbf{r} \in \tilde{\Omega},
\end{aligned}
$$


where $u_{n, h}^{(k)}=\left.\mathbf{u}_{h}^{(k)} \cdot \mathbf{n}\right|_{\partial \tilde{\Omega}}$ and $\chi_{h}^{(0)}=\chi_{h}^{\|}$and $\mathbf{u}_{h}^{(0)}=\mathbf{j}_{h}^{\|}$. In Appendix D, all the details of the derivation of radiation corrections for plasmonic resonances and modes are reported. Here only the main results are shown. In the following, the EQS current modes are normalized accordingly to Eq. (4), i.e. $\|\mathbf{j}\|_{h}^{\|} \|=1, \forall h$.

Matching the first-order terms in Eq. (26), it is obtained that the first order corrections vanish regardless of the object's shape:

$$
\begin{gathered}
\chi_{h}^{(1)}=0, \\
\mathbf{u}_{h}^{(1)}(\tilde{\mathbf{r}})=\mathbf{0} \quad \forall \mathbf{r} \in \tilde{\Omega} .
\end{gathered}
$$

Collecting the second order terms in Eq. (26), and applying the normal solvability condition of Fredholm integral equations $[61,62]$, the second order correction $\chi_{h}^{(2)}$ is derived:

$$
\chi_{h}^{(2)}=-\left(\chi_{h}^{\|}\right)^{2} \frac{1}{4 \pi}\left(\oint_{\partial \tilde{\Omega}} j_{n, h}^{\|}(\tilde{\mathbf{r}}) \oint_{\partial \tilde{\Omega}} \frac{\left|\tilde{\mathbf{r}}-\tilde{\mathbf{r}}^{\prime}\right|}{2} j_{n, h}^{\|}\left(\tilde{\mathbf{r}}^{\prime}\right) d \tilde{S}^{\prime} d \tilde{S}+\int_{\tilde{\Omega}} \mathbf{j}_{h}^{\|}(\tilde{\mathbf{r}}) \cdot \int_{\tilde{\Omega}} \frac{\mathbf{j}_{h}^{\|}\left(\tilde{\mathbf{r}}^{\prime}\right)}{\left|\tilde{\mathbf{r}}-\tilde{\mathbf{r}}^{\prime}\right|} d \tilde{V}^{\prime} d \tilde{V}\right),
$$

where $j_{n, h}^{\|}(\tilde{\mathbf{r}})=\left.\mathbf{j}_{h}^{\|}(\tilde{\mathbf{r}}) \cdot \hat{\mathbf{n}}(\tilde{\mathbf{r}})\right|_{\partial \tilde{\Omega}}$ is a scalar field defined on the object's surface $\partial \tilde{\Omega}$. Accordingly to Eq. (29), $\chi_{h}^{(2)}$ is real. Moreover, the first term in parenthesis in Eq. (29) (starting from the left) originates from the radiative self-interaction of the surface charge density associated to the EQS current mode through the scalar potential. The second term is instead proportional to the magnetostatic energy of the current mode $\mathbf{j}_{h}^{\|}$. A second order correction to the EQS modes has been already derived in Ref. [51] by expanding the Maxwell's equation in differential form. It will be demonstrated in Eq. (59) that $\chi_{h}^{(2)}$ is associated with the frequency-shift of the $h$-th plasmonic mode.

The second order correction of the associated plasmonic mode $\mathbf{u}_{h}^{(2)}$ has both longitudinal and transverse components, denoted as $\mathbf{u}_{h}^{(2) \|}$ and $\mathbf{u}_{h}^{(2) \perp}$, respectively:

$$
\mathbf{u}_{h}^{(2)}=\mathbf{u}_{h}^{(2) \|}+\mathbf{u}_{h}^{(2) \perp}=\sum_{\substack{k=1 \\ k \neq h}}^{\infty} \alpha_{h, k}^{(2)} \mathbf{j}_{k}^{\|}+\sum_{k=1}^{\infty} \beta_{h, k}^{(2)} \mathbf{j}_{k}^{\perp},
$$

where the longitudinal part $\mathbf{u}_{h}^{(2) \|}$ is represented in terms of the EQS modes basis $\left\{\mathbf{j}_{k}^{\|}\right\}_{k \in \mathbb{N}}$, and the transverse part $\mathbf{u}_{h}^{(2) \perp}$ in terms of the MQS modes basis $\left\{\mathbf{j}_{k}^{\perp}\right\}_{k \in \mathbb{N}}$. The expansion coefficients are:

$$
\begin{aligned}
& \alpha_{h, k}^{(2)}=\frac{1}{4 \pi} \frac{\chi_{k}^{\|} \chi_{h}^{\|}}{\chi_{k}^{\|}-\chi_{h}^{\|}}\left(\oint_{\partial \tilde{\Omega}} j_{n, h}^{\|}(\tilde{\mathbf{r}}) \oint_{\partial \tilde{\Omega}} \frac{\left|\tilde{\mathbf{r}}-\tilde{\mathbf{r}}^{\prime}\right|}{2} j_{n, k}^{\|}\left(\tilde{\mathbf{r}}^{\prime}\right) d \tilde{S}^{\prime} d \tilde{S}+\int_{\tilde{\Omega}} \mathbf{j}_{h}^{\|}(\tilde{\mathbf{r}}) \cdot \int_{\tilde{\Omega}} \frac{\mathbf{j}_{k}^{\|}\left(\tilde{\mathbf{r}}^{\prime}\right)}{\left|\tilde{\mathbf{r}}-\tilde{\mathbf{r}}^{\prime}\right|} d \tilde{V}^{\prime} d \tilde{V}\right), \quad \forall k \neq h \\
& \beta_{h, k}^{(2)}=\frac{1}{4 \pi} \chi_{h}^{\|} \int_{\tilde{\Omega}} \mathbf{j}_{h}^{\|}(\tilde{\mathbf{r}}) \cdot \int_{\tilde{\Omega}} \frac{\mathbf{j}_{k}^{\perp}\left(\tilde{\mathbf{r}}^{\prime}\right)}{\left|\tilde{\mathbf{r}}-\tilde{\mathbf{r}}^{\prime}\right|} d \tilde{V}^{\prime} d \tilde{V}, \quad \forall k \in \mathbb{N} .
\end{aligned}
$$

Matching the third order terms in Eq. (26), the third order correction $\chi_{h}^{(3)}$ is obtained:

$$
\chi_{h}^{(3)}=i \frac{1}{6 \pi}\left(\chi_{h}^{\|}\right)^{2}\left\|\mathbf{P}_{\mathrm{E}, h}^{\|}\right\|^{2},
$$

which is purely imaginary and proportional to the squared norm of the dipole moment $\mathbf{P}_{\mathrm{E}, h}^{\|}$of the $h$-th EQS mode.

As it will be demonstrated in Eq. (61), $\chi_{h}^{(3)}$ determines the radiative Q-factor of $h$-th plasmonic mode. However, for dark modes $\chi_{h}^{(3)}$ vanishes. In this case, to retrieve information about the radiative Q-factor, it is mandatory to consider the fifth order perturbation $\chi_{h}^{(5)}$. For dark modes, it can be expressed in terms of the electric quadrupole tensor $\overleftrightarrow{\mathbf{Q}}_{\mathrm{E} \mid h}^{\|}$of the $h$-th EQS mode, and its components $Q_{\mathrm{E}|h| i j}^{\|}$:

$$
\chi_{h}^{(5)}=i \frac{1}{80 \pi}\left(\chi_{h}^{\|}\right)^{2}\left[\sum_{i j}\left(Q_{\mathrm{E}|h| i j}^{\|}\right)^{2}-\frac{1}{3}\left(\operatorname{Tr} \overleftrightarrow{\mathbf{Q}}_{\mathrm{E}}^{\|}\right)^{2}\right]
$$

where Tr is the trace operator, and $\overleftrightarrow{\mathbf{Q}}_{\mathrm{E} \mid h}^{\|}$is defined by Eq. (A2) of the Appendix A. Thus, the fifth order correction is purely imaginary and it is related to the power radiated to infinity by the electric quadrupole.

The outlined procedure can be iteratively applied: if the fifth order correction vanishes, the next order correction that may give an imaginary contribution is the seventh, which can be calculated by matching the terms of corresponding-order in Eq. (26).

\section{DIELECTRIC MODES AND RESONANCES}

To evaluate the dielectric resonances beyond the quasistatic regime it is convenient to recast the eigenvalue problem (20) as

$$
\begin{array}{r}
-x^{2} \mathbf{v}_{h}(\tilde{\mathbf{r}})-\kappa_{h} \tilde{\nabla} \oint_{\partial \tilde{\Omega}} g\left(\tilde{\mathbf{r}}-\tilde{\mathbf{r}}^{\prime}, x\right) \mathbf{v}_{h}\left(\tilde{\mathbf{r}}^{\prime}\right) \cdot \hat{\mathbf{n}}\left(\tilde{\mathbf{r}}^{\prime}\right) d \tilde{S}^{\prime} \\
+\kappa_{h} x^{2} \int_{\tilde{\Omega}} g\left(\tilde{\mathbf{r}}-\tilde{\mathbf{r}}^{\prime}, x\right) \mathbf{v}_{h}\left(\tilde{\mathbf{r}^{\prime}}\right) d \tilde{V}^{\prime}=\mathbf{0} \quad \forall \tilde{\mathbf{r}} \in \tilde{\Omega} .
\end{array}
$$


The Green function $g\left(\tilde{\mathbf{r}}-\tilde{\mathbf{r}}^{\prime}, x\right)$, the mode $\mathbf{v}_{h}$ and the corresponding eigenvalue $\kappa_{h}$ are expanded at $x=0$ in the neighborhood of MQS eigenvalue $\kappa_{h}^{\perp}$ and mode $\mathbf{j}_{h}^{\perp}$ :

$$
\begin{gathered}
\kappa_{h}=\kappa_{h}^{\perp}+\kappa_{h}^{(1)} x+\kappa_{h}^{(2)} x^{2}+\ldots=\sum_{k=0}^{\infty} \kappa_{h}^{(k)} x^{k}, \\
\mathbf{v}_{h}=\mathbf{j}_{h}^{\perp}+\mathbf{v}_{h}^{(1)} x+\mathbf{v}_{h}^{(2)} x^{2}+\cdots=\sum_{k=0}^{\infty} \mathbf{v}_{h}^{(k)} x^{k} .
\end{gathered}
$$

By substituting Eqs. (36), (37), and (25) in Eq. (35) the following equation is obtained:

$$
\begin{aligned}
& -4 \pi \sum_{k=0}^{\infty} \mathbf{v}_{h}^{(k)} x^{k+2}-\left(\sum_{k=0}^{\infty} \kappa_{h}^{(k)} x^{k}\right) \tilde{\nabla} \oint_{\partial \tilde{\Omega}}\left(\sum_{k=0}^{\infty}(-i)^{k} \frac{\Delta \tilde{\mathbf{r}}^{k-1}}{k !} x^{k}\right)\left(\sum_{k=0}^{\infty} v_{h n}^{(k)} x^{k}\right) d \tilde{S}^{\prime} \\
& +\left(\sum_{k=0}^{\infty} \kappa_{h}^{(k)} x^{k}\right) \int_{\tilde{\Omega}}\left(\sum_{k=0}^{\infty}(-i)^{k} \frac{\Delta \tilde{\mathbf{r}}^{k-1}}{k !} x^{k}\right)\left(\sum_{k=0}^{\infty} \mathbf{v}_{h}^{(k)} x^{k+2}\right) d \tilde{V}^{\prime}=0, \quad \forall \mathbf{r} \in \tilde{\Omega},
\end{aligned}
$$

where $v_{h n}^{(k)}=\left.\mathbf{v}_{h}^{(k)} \cdot \mathbf{n}\right|_{\partial \Omega}, \kappa_{h}^{(0)}=\kappa_{h}^{\perp}$ and $\mathbf{v}_{h}^{(0)}=\mathbf{j}_{h}^{\perp}$. In Appendix $\mathrm{E}$, all the details on the derivation of radiation corrections for dielectric resonances are reported. Here, only the main results are highlighted. In the following, the MQS are normalized accordingly to Eq. (12), i.e., $\left\|\mathbf{j}_{h}^{\perp}\right\|=1$ $\forall h$.

By matching the terms of corresponding order in Eq. (38), it is possible to demonstrate that first-order corrections vanish regardless of the shape of the object:

$$
\begin{aligned}
\kappa_{h}^{(1)} & =0, \\
\mathbf{v}_{h}^{(1)}(\tilde{\mathbf{r}}) & =0, \quad \forall \mathbf{r} \in \tilde{\Omega} .
\end{aligned}
$$

The second-order correction $\kappa_{h}^{(2)}$ is a real quantity, namely,

$$
\begin{aligned}
\kappa_{h}^{(2)}= & \frac{\left(\kappa_{h}^{\perp}\right)^{2}}{4 \pi}\left[\int_{\tilde{\Omega}} \mathbf{j}_{h}^{\perp}(\tilde{\mathbf{r}}) \cdot \int_{\tilde{\Omega}} \frac{\left|\tilde{\mathbf{r}}-\tilde{\mathbf{r}}^{\prime}\right|}{2} \mathbf{j}_{h}^{\perp}\left(\tilde{\mathbf{r}}^{\prime}\right) d \tilde{V}^{\prime} d \tilde{V}\right. \\
& \left.+\sum_{k=1}^{\infty} \frac{\chi_{k}^{\|}}{4 \pi}\left|\int_{\tilde{\Omega}} \mathbf{j}_{k}^{\|}(\tilde{\mathbf{r}}) \cdot \int_{\tilde{\Omega}} \frac{\mathbf{j}_{h}^{\perp}\left(\tilde{\mathbf{r}}^{\prime}\right)}{\left|\tilde{\mathbf{r}}-\tilde{\mathbf{r}}^{\prime}\right|} d \tilde{V}^{\prime} d \tilde{V}\right|^{2}\right] .
\end{aligned}
$$

The first term in parentheses in Eq. (41) originates from the radiative self-interaction of the MQS mode $\mathbf{j}_{h}^{\perp}$ through the vector potential. The second terms is a summation, where each addend is proportional to the ratio of the magnetostatic interaction energy between the MQS current mode $\mathbf{j}_{h}^{\perp}$ and the EQS current mode $\mathbf{j}_{k}^{\|}$, i.e.,

$$
\mathscr{W}_{m \mathrm{I}}\left\{\mathbf{j}_{k}^{\|}, \mathbf{j}_{h}^{\perp}\right\}=\frac{\mu_{0}}{8 \pi} \int_{\tilde{\Omega}} \mathbf{j}_{k}^{\|}(\tilde{\mathbf{r}}) \cdot \int_{\tilde{\Omega}} \frac{\mathbf{j}_{h}^{\perp}\left(\tilde{\mathbf{r}}^{\prime}\right)}{\left|\tilde{\mathbf{r}}-\tilde{\mathbf{r}}^{\prime}\right|} d \tilde{V}^{\prime} d \tilde{V},
$$

to the electrostatic energy of the EQS current mode $\mathbf{j}_{k}^{\|}$[using Eq. (6)]. The MQS current mode $\mathbf{j}_{h}^{\perp}$ may be an $\mathbb{A}^{\perp}$ mode, generating a transverse vector potential, according to the definition (15). In this case, since every EQS current mode is longitudinal, and transverse and longitudinal functions are orthogonal according to the scalar product (5), the energy $\mathscr{W}_{m \mathrm{I}}\left\{\mathbf{j}_{k}^{\|}, \mathbf{j}_{h}^{\perp}\right\}$ vanishes $\forall k$, and Eq. (41) further simplifies:

$$
\begin{array}{r}
\kappa_{h}^{(2)}=\frac{\left(\kappa_{h}^{\perp}\right)^{2}}{4 \pi} \int_{\tilde{\Omega}} \mathbf{j}_{h}^{\perp}(\tilde{\mathbf{r}}) \cdot \int_{\tilde{\Omega}} \frac{\left|\tilde{\mathbf{r}}-\tilde{\mathbf{r}}^{\prime}\right|}{2} \mathbf{j}_{h}^{\perp}\left(\tilde{\mathbf{r}}^{\prime}\right) d \tilde{V}^{\prime} d \tilde{V} \\
\text { for } \mathbb{A}^{\perp} \text { modes. }
\end{array}
$$

As will be demonstrated in Eq. (69), $\kappa_{h}^{(2)}$ is associated with the frequency shift of dielectric modes.

The second-order correction $\mathbf{v}_{h}^{(2)}$ to the current density mode has both longitudinal and transverse components, denoted as $\mathbf{v}_{h}^{(2) \|}$ and $\mathbf{v}_{h}^{(2) \perp}$, which can be in turn expanded in terms of EQS and MQS current modes, respectively:

$$
\mathbf{v}_{h}^{(2)}=\mathbf{v}_{h}^{(2) \|}+\mathbf{v}_{h}^{(2) \perp}=\sum_{k=1}^{\infty} \alpha_{h, k}^{(2)} \mathbf{j}_{k}^{\|}+\sum_{\substack{k=1 \\ k \neq h}}^{\infty} \beta_{h, k}^{(2)} \mathbf{j}_{k}^{\perp},
$$

where

$$
\begin{aligned}
& \alpha_{h, k}^{(2)}=-\frac{\chi_{k}^{\|}}{4 \pi} \int_{\tilde{\Omega}} \mathbf{j}_{k}^{\|}(\tilde{\mathbf{r}}) \cdot \int_{\tilde{\Omega}} \frac{\mathbf{j}_{h}^{\perp}\left(\tilde{\mathbf{r}}^{\prime}\right)}{\left|\tilde{\mathbf{r}}-\tilde{\mathbf{r}}^{\prime}\right|} d \tilde{V}^{\prime} d \tilde{V}, \quad \forall k, \\
& \beta_{h, k}^{(2)}=\frac{\kappa_{k}^{\perp} \kappa_{h}^{\perp}}{\kappa_{h}^{\perp}-\kappa_{k}^{\perp}} \frac{1}{4 \pi}\left[\int_{\tilde{\Omega}} \mathbf{j}_{k}^{\perp}(\tilde{\mathbf{r}}) \cdot \int_{\tilde{\Omega}} \frac{\left|\tilde{\mathbf{r}}-\tilde{\mathbf{r}}^{\prime}\right|}{2} \mathbf{j}_{h}^{\perp}\left(\tilde{\mathbf{r}}^{\prime}\right) d \tilde{V}^{\prime} d \tilde{V}-\sum_{s=1}^{\infty} \alpha_{h, s}^{(2)} \int_{\tilde{\Omega}} \mathbf{j}_{s}^{\|}\left(\tilde{\mathbf{r}}^{\prime}\right) \cdot \int_{\tilde{\Omega}} \frac{\mathbf{j}_{k}^{\perp}(\tilde{\mathbf{r}})}{\left|\tilde{\mathbf{r}}-\tilde{\mathbf{r}}^{\prime}\right|} d \tilde{V}^{\prime} d \tilde{V}\right], \quad \forall k \neq h .
\end{aligned}
$$


Although any MQS mode has a zero electric dipole moment, its second-order radiative correction $\mathbf{v}_{h}^{(2)}$ may exhibit a nonzero electric dipole moment $\mathbf{P}_{\mathrm{E} \mid h}^{(2)}$, given by

$$
\mathbf{P}_{\mathrm{E} \mid h}^{(2)}=\sum_{k=1}^{\infty} \alpha_{h, k}^{(2)} \mathbf{P}_{\mathrm{E} \mid k}^{\|}
$$

where $\mathbf{P}_{\mathrm{E} \mid k}^{\|}$is the electric dipole moment of the $k$ th EQS mode $\mathbf{j}_{k}^{\|}$. For $\mathbb{A}^{\perp}$ modes, the longitudinal part of $\mathbf{v}_{h}^{(2)}$ vanishes, and they do not display electric dipole moment up to this order.

The third-order correction $\kappa_{h}^{(3)}$ is purely imaginary and depends on the magnetic dipole moment $\mathbf{P}_{\mathrm{M}, h}^{\perp}$ of the mode $\mathbf{j}_{h}^{\perp}$ :

$$
\kappa_{h}^{(3)}=i\left(\kappa_{h}^{\perp}\right)^{2} \frac{1}{6 \pi}\left\|\mathbf{P}_{\mathrm{M}, h}^{\perp}\right\|^{2},
$$

where $\mathbf{P}_{\mathrm{M}, h}^{\perp}$ is defined in Eq. (A3). The third-order correction to the mode, i.e., $\mathbf{v}_{h}^{(3)}$, is purely transverse and thus can be expanded in the basis of MQS modes

$$
\mathbf{v}_{h}^{(3)}=\mathbf{v}_{h}^{(3) \perp}=\sum_{\substack{k=1 \\ k \neq h}}^{\infty} \beta_{h, k}^{(3)} \mathbf{j}_{k}^{\perp},
$$

where the expansion coefficient $\beta_{h, k}^{(3)}$ depends on the dot product of the magnetic dipole moments $\mathbf{P}_{\mathrm{M} \mid h}^{\perp}$ and $\mathbf{P}_{\mathrm{M} \mid k}^{\perp}$ of the $h$ th and $k$ th MQS modes:

$$
\beta_{h, k}^{(3)}=i \frac{1}{6 \pi} \frac{\kappa_{k}^{\perp} \kappa_{h}^{\perp}}{\kappa_{h}^{\perp}-\kappa_{k}^{\perp}} \mathbf{P}_{\mathrm{M} \mid h}^{\perp} \cdot \mathbf{P}_{\mathrm{M} \mid k}^{\perp} \quad \forall h \neq k .
$$

As will be shown in Eq. (71), the correction $\kappa_{h}^{(3)}$, if nonvanishing, determines the radiative Q-factor of the $h$ th dielectric mode.

However, $\kappa_{h}^{(3)}$ vanishes when the corresponding magnetic dipole moment is zero. In this case, the next imaginary correction has order 5 and has the following expression:

$$
\kappa_{h}^{(5)}=i \frac{\left(\kappa_{h}^{\perp}\right)^{2}}{80 \pi} \sum_{i j}\left(Q_{\mathrm{M}|h| i j}^{\perp}\right)^{2}+i \frac{\left(\kappa_{h}^{\perp}\right)^{2}}{6 \pi}\left\|\mathbf{P}_{\mathrm{E} 2 \mid h}^{\perp}-\mathbf{P}_{\mathrm{E} \mid h}^{(2)}\right\|^{2},
$$

where $\overleftrightarrow{\mathbf{Q}}_{\mathrm{M} \mid h}^{\perp}$ is the magnetic quadrupole tensor of the $h$ th MQS mode, introduced in Eq. (A5), and $Q_{\mathrm{M}|h| i j}^{\perp}$ are its components, $\mathbf{P}_{\mathrm{E} 2 \mid h}^{\perp}$ is the toroidal dipole defined in Eq. (A4), and $\mathbf{P}_{\mathrm{E} \mid h}^{(2)}$ is the electric dipole moment of the second-order correction $\mathbf{v}_{h}^{(2)}$, introduced in Eq. (47). In conclusion, the fifth order correction depends on the power radiated to infinity by the magnetic quadrupole $\overleftrightarrow{\mathbf{Q}}_{\mathbf{M} \mid h}^{\perp}$ and by an effective electric dipole resulting from the interference between the $\mathbf{P}_{\mathrm{E} 2 \mid h}^{\perp}$ and $\mathbf{P}_{\mathrm{E} \mid h}^{(2)}$.

For $\mathbb{A}^{\perp}$ modes, Eq. (51) further simplifies:

$$
\kappa_{h}^{(5)}=i\left(\kappa_{h}^{\perp}\right)^{2}\left\{\frac{1}{80 \pi} \sum_{i j}\left(Q_{\mathrm{M}|h| i j}^{\perp}\right)^{2}+\frac{1}{6 \pi}\left\|\mathbf{P}_{\mathrm{E} 2 \mid h}^{\perp}\right\|^{2}\right\} \text {. }
$$

The outlined procedure can be iteratively applied. If the fifth-order correction vanishes, the next order correction that may give an imaginary contribution is the seventh, which can be calculated by matching the terms of ninth order in Eq. (38).

\section{RESONANCE FREQUENCY AND Q-FACTOR}

In the previous section, the second-order corrections $\chi_{h}^{(2)}$, $\kappa_{h}^{(2)}$ and nonvanishing imaginary corrections $\chi_{h}^{\left(n_{i}\right)}, \kappa_{h}^{\left(n_{i}\right)}$ of the lowest order, called $n_{i}$, are derived in closed form for both plasmonic and dielectric modes. They depend neither on the size of the object nor on its permittivity, but only on the morphology of the EQS and MQS modes. In this section, closed-form expressions of the resonance frequency and Qfactors are obtained in terms of these corrections for both metal and dielectric objects. The modes are assumed to be noninteracting. Moreover, throughout this work, we use the definition of Q-factor as the inverse of the $-3-\mathrm{dB}$ fractional bandwidth.

\section{A. Plasmonic resonances}

It is now assumed that the object is made of a timedispersive metal described by the Drude model $[63,64]$,

$$
\chi(\omega)=-\frac{\omega_{p}^{2}}{\omega(\omega-i \nu)},
$$

where $\omega_{p}$ and $v$ are the plasma and collision angular frequencies, and $v \ll \omega_{p}$. It is also useful to define the quantity

$$
x_{p}=\frac{\omega_{p}}{c_{0}} l_{c}=2 \pi \frac{l_{c}}{\lambda_{p}},
$$

where $\lambda_{p}$ is the plasma wavelength. The EQS resonance frequency $\omega_{h}^{\|}$of the $h$ th EQS mode $\mathbf{j}_{h}^{\|}$is defined as the frequency at which the real part of the metal susceptibility $\operatorname{Re}\{\chi(\omega)\}$ matches the EQS eigenvalue $\chi_{h}^{\|}$, i.e.,

$$
\frac{x_{h}^{\|}}{x_{p}}=\frac{\omega_{h}^{\|}}{\omega_{p}}=\frac{1}{\sqrt{-\chi_{h}^{\|}}},
$$

where $x_{h}^{\|}=\left(\omega_{h}^{\|} / c_{0}\right) l_{c}$ is the size parameter at the EQS resonance.

In the full-wave scenario, the resonance of the $h$ th plasmonic mode $\mathbf{u}_{h}$ (which tends to $\mathbf{j}_{h}^{\|}$in the quasistatic limit) is defined by setting to zero the real part of the denominator of the $h$ th addend of the first summation in Eq. (21). Thus, the value $x_{h}$ of the size parameter at the plasmonic resonance is the value of $x$ at which the real part of the metal susceptibility $\chi(\omega)$ matches the real part of the corresponding eigenvalue $\chi_{h}\left(x_{h}\right)$ of Eq. (20), i.e.,

$$
\operatorname{Re}\left\{\chi_{h}\right\}=\operatorname{Re}\left\{\chi\left(\omega_{h}\right)\right\} \approx-\frac{\omega_{p}^{2}}{\omega_{h}^{2}}=-\frac{x_{p}^{2}}{x_{h}^{2}},
$$

and $\omega_{h}$ is the corresponding resonance frequency. Equation (56) is the resonance condition of the plasmonic modes. For small particles $x_{p} \lesssim 1$, by retaining only the real and imaginary nonzero corrections of the lowest order in Eq. (23), the plasmonic eigenvalue $\chi_{h}(x)$ is approximated as

$$
\chi_{h}(x) \approx \chi_{h}^{\|}+\chi_{h}^{(2)} x^{2}+\chi_{h}^{\left(n_{i}\right)} x^{n_{i}},
$$


where $n_{i}$ is the order of the first nonzero imaginary correction $\chi_{h}^{\left(n_{i}\right)}$. By using Eq. (57) in (56), and solving the resulting biquadratic equation, the resonance frequency is obtained:

$$
\frac{\omega_{h}}{\omega_{p}}=\frac{x_{h}}{x_{p}}=\frac{1}{\sqrt{2} x_{p}} \sqrt{\frac{\chi_{h}^{\|}}{\chi_{h}^{(2)}}\left(\sqrt{1-4 \frac{\chi_{h}^{(2)}}{\left(\chi_{h}^{\|}\right)^{2}} x_{p}^{2}}-1\right)} .
$$

In the limit $x_{p} \ll 1$, the frequency shift of the plasmonic resonance with respect to the EQS resonance, i.e., $\Delta \omega_{h}=$ $\omega_{h}-\omega_{h}^{\|}$, and the corresponding shift in the resonance size parameter, i.e., $\Delta x_{h}=x_{h}-x_{h}^{\|}$, can be approximated as

$$
\frac{\Delta \omega_{h}}{\omega_{h}^{\|}}=\frac{\Delta x_{h}}{x_{h}^{\|}} \approx-\frac{1}{2} \frac{\chi_{h}^{(2)}}{\chi_{h}^{\|}}\left(x_{h}^{\|}\right)^{2}, \quad x_{p} \ll 1 .
$$

In conclusion, the relative frequency shift of any plasmonic mode is a quadratic function of $x_{h}^{\|}$, whose prefactor is one half the ratio between the second-order correction $\chi_{h}^{(2)}$ and the EQS eigenvalue $\chi_{h}^{\|}$.

The radiative $\mathrm{Q}$-factor $\mathrm{Q}_{h}^{\| \mathrm{r}}$ of the $h$ th plasmonic mode is obtained by considering the inverse of the fractional bandwidth of the $h$ th addend of the first summation in Eq. (21), assuming negligible nonradiative losses, and using the expansion (57):

$$
\mathrm{Q}_{h}^{\| \mathrm{r}} \approx\left|\frac{\chi_{h}^{\|}}{\chi_{h}^{\left(n_{i}\right)}}\right|\left(\frac{1}{x_{h}}\right)^{n_{i}} .
$$

The Q-factor is an inverse power function of the size parameter at the resonance, whose exponent is the order $n_{i}$ of the first nonvanishing imaginary correction $\chi_{h}^{\left(n_{i}\right)}$, while the prefactor is the ratio between the EQS eigenvalue $\chi_{h}^{\|}$and the correction $\chi_{h}^{\left(n_{i}\right)}$.

In Fig. 1, the algorithm for the calculation of the radiative Q-factor of any plasmonic mode is summarized by a flowchart. First, the EQS current modes and corresponding eigenvalues are computed by solving the eigenvalue problem (1). Thus, if the $h$ th mode is bright, namely it exhibits a nonvanishing electric dipole moment $\mathbf{P}_{\mathrm{E} \mid h}^{\|}$, it follows that $\chi_{h}^{(3)} \neq 0$, $n_{i}=3$, and the Q-factor is obtained by combining Eqs. (33) and (60):

$$
\mathrm{Q}_{h}^{\| \mathrm{r}} \approx \frac{6 \pi}{\chi_{h}^{\|}\left\|\mathbf{P}_{\mathrm{E} \mid h}^{\|}\right\|^{2}}\left(\frac{1}{x_{h}}\right)^{3}
$$

By expressing in Eq. (61) the EQS eigenvalue $\chi_{h}^{\|}$in terms of the electrostatic energy $\mathscr{W}_{e}\left\{\mathbf{j}_{h}^{\|}\right\}$of the mode $\mathbf{j}_{h}^{\|}$[using Eq. (6)], $\mathrm{Q}_{h}^{\| \mathrm{r}}$ is also found equal to $2 \pi$ times the ratio of $\mathscr{W}_{e}\left\{\mathbf{j}_{h}^{\|}\right\}$to the energy radiated to infinity in a period by the electric dipole $\mathbf{P}_{\mathrm{E} \mid h}^{\|}$at the resonance frequency $\omega_{h}$.

Instead, for a dark mode with nonvanishing electric quadrupole tensor, it follows that $\chi_{h}^{(5)} \neq 0, n_{i}=5$, and the Q-factor is determined by combining Eqs. (34) and (60),

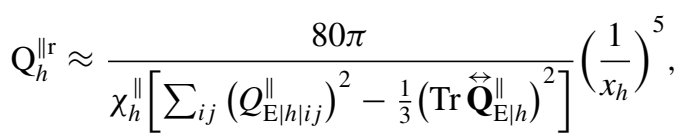

which is also equal to $2 \pi$ times the ratio of the electrostatic energy of the $h$ th EQS mode to the energy radiated to infinity in a period by the electric quadrupole $\overleftrightarrow{\mathbf{Q}}_{\mathrm{E} \mid h}^{\|}$at the resonance frequency $\omega_{h}$. If the electric quadrupole moment is also vanishing, the outlined process can be iterated by considering higher order electric multipoles.

For completeness, we also consider the opposite regime, dominated by nonradiative losses. In this case, the nonradiative $\mathrm{Q}$-factor $\mathrm{Q}_{h}^{\| \mathrm{nr}}$ is obtained as the inverse of the fractional bandwidth of the $h$ th addend of the first summation in Eq. (21), assuming negligible radiative losses. It has the expression

$$
\mathrm{Q}_{h}^{\| \mathrm{nr}}=\frac{\omega_{h}}{v} \approx \frac{\omega_{p}}{v \sqrt{-\chi}} .
$$

Equation (63) is not new, but it was already shown by Wang and Shen in Ref. [4].

In an intermediate regime, the resulting Q-factor, indicated with $\mathrm{Q}_{h}^{\|}$, can be obtained as [65]

$$
\frac{1}{\mathrm{Q}_{h}^{\|}}=\frac{1}{\mathrm{Q}_{h}^{\| \mathrm{r}}}+\frac{1}{\mathrm{Q}_{h}^{\| \mathrm{nr}}} \text {. }
$$

\section{B. Dielectric resonances}

It is now assumed that the object is made of a nondispersive dielectric material with positive susceptibility $\chi \geqslant 0$, with $\operatorname{Im}\{\chi\} \ll \operatorname{Re}\{\chi\}$. The size parameter $x_{h}^{\perp}=\left(\omega_{h}^{\perp} / c_{0}\right) l_{c}$ at the resonance of the $h$ th MQS mode $\mathbf{j}_{h}^{\perp}$ is defined as the value of $x$ at which the real part of the susceptibility $\chi$ matches the eigenvalue $\kappa_{h}^{\perp} / x^{2}$, namely,

$$
x_{h}^{\perp}=\frac{\omega_{h}^{\perp}}{c_{0}} l_{c}=\sqrt{\frac{\kappa_{h}^{\perp}}{\operatorname{Re}\{\chi\}}},
$$

and $\omega_{h}^{\perp}$ is the corresponding MQS resonance frequency.

In the full-wave regime, the resonance of the $h$ th dielectric mode $\mathbf{v}_{h}$ (which tends to $\mathbf{j}_{h}^{\perp}$ in the quasistatic limit) is defined by setting to zero the real part of the denominator of the $h$ th addend of the second summation in Eq. (21). Thus, the value of size parameter $x_{h}=\left(\omega_{h} / c_{0}\right) l_{c}$ at the dielectric resonance is the value of $x$ at which the real part of the eigenvalue $\kappa_{h} / x^{2}$ matches the quantity $\operatorname{Re}\{\chi\}$

$$
\operatorname{Re}\left\{\kappa_{h}\right\}=\operatorname{Re}\{\chi\} x^{2} .
$$

This is the resonance condition for dielectric modes, and $\omega_{h}$ is the dielectric resonance frequency. For small particles $x \lesssim 1$, by keeping only the real and imaginary nonzero corrections of the lowest order in Eq. (36), the dielectric eigenvalue $\kappa_{h}(x)$ is approximated as

$$
\kappa(x) \approx \kappa_{h}^{\perp}+\kappa_{h}^{(2)} x^{2}+\kappa_{h}^{\left(n_{i}\right)} x^{n_{i}},
$$

where $n_{i}$ is the order of the first nonzero imaginary correction $\kappa_{h}^{\left(n_{i}\right)}$. By using Eq. (67) in (66) and solving the resulting quadratic equation, the resonance size parameter is obtained:

$$
x_{h}=\frac{\omega_{h}}{c_{0}} l_{c} \approx \sqrt{\frac{\kappa_{h}^{\perp}}{\operatorname{Re}\{\chi\}-\kappa_{h}^{(2)}}}=\frac{x_{h}^{\perp}}{\sqrt{1-\kappa_{h}^{(2)} / \operatorname{Re}\{\chi\}}} .
$$

For high-index dielectrics $\operatorname{Re}\{\chi\} \gg 1$, the relative frequency shift of the $h$ th dielectric resonance with respect to the MQS 


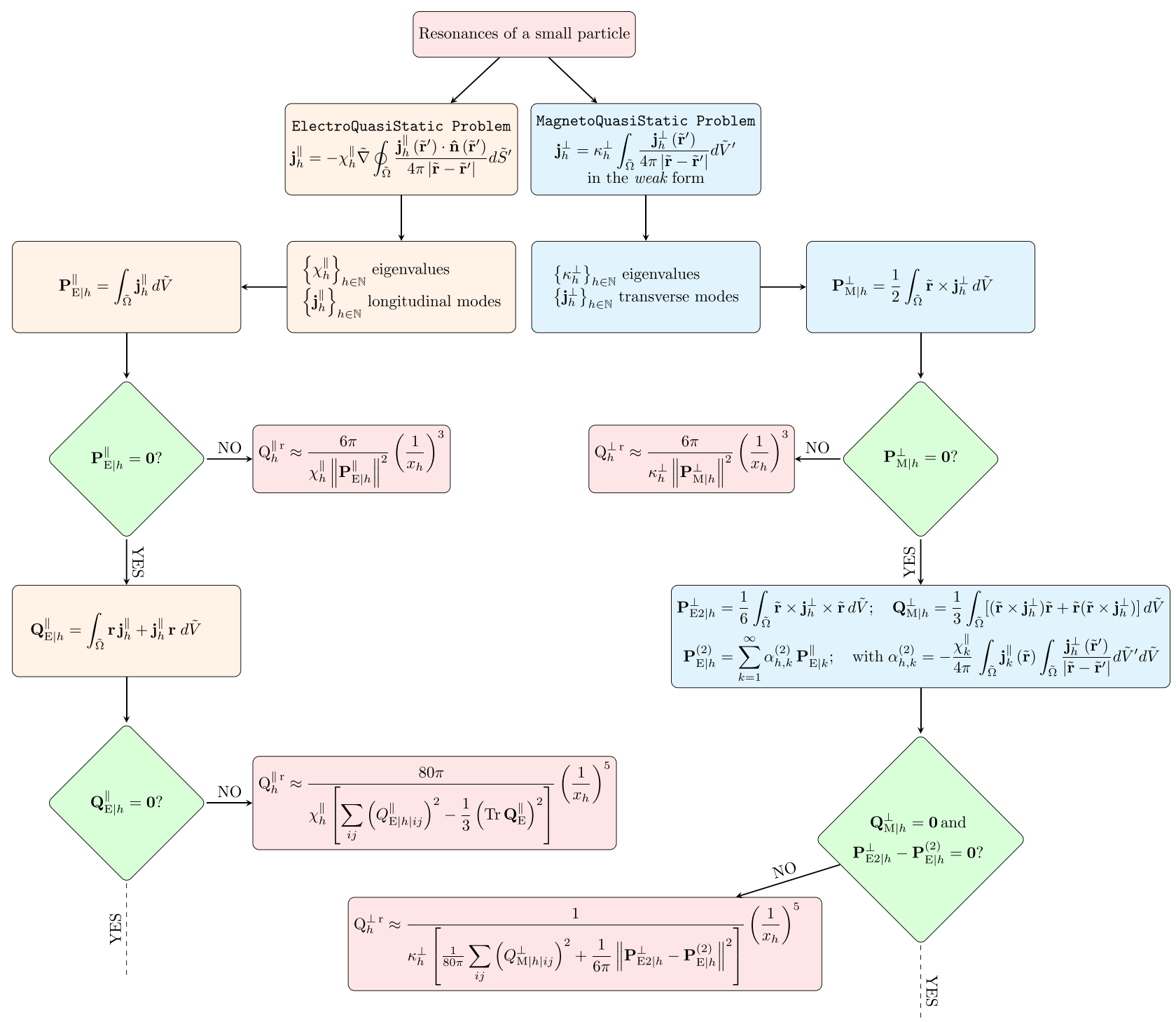

FIG. 1. Algorithm to compute the radiative Q-factor of either plasmonic or dielectric resonances of a small homogeneous isotropic nonmagnetic object. In the plasmonic case, the EQS current modes and corresponding eigenvalues are computed. If the $h$ th EQS mode is bright with nonvanishing electric dipole moment $\mathbf{P}_{\mathrm{E} \mid h}^{\|}$, its radiative Q-factor is given by Eq. (61). Instead, for a dark mode with nonvanishing electric quadrupole tensor $\overleftrightarrow{\mathbf{Q}}_{\mathrm{E} \mid h}^{\|}$, the Q-factor is given by (62). If $\overleftrightarrow{\mathbf{Q}}_{\mathrm{E} \mid h}^{\|}$is also vanishing, the outlined process can be iterated by considering higher order electric multipoles. In the dielectric case, the MQS current modes and corresponding eigenvalues are computed. Thus, if the $h$ th MQS mode exhibits a nonvanishing magnetic dipole moment $\mathbf{P}_{\mathrm{M} \mid h}^{\perp}$, the radiative Q-factor is given by Eq. (71). Instead, if the magnetic dipole vanishes, but at least one among the magnetic quadrupole $\overleftrightarrow{\mathbf{Q}}_{\mathrm{M}|h| i j}^{\perp}$, and the effective dipole moment resulting from the difference of the toroidal dipole $\mathbf{P}_{\mathrm{E} 2 \mid h}^{\perp}$ and of the electric dipole moment of the second-order mode correction $\mathbf{P}_{\mathrm{E} \mid h}^{(2)}$ is nonvanishing, the radiative Q-factor is given by Eq. (72). If they are all vanishing, the outlined algorithm has to be iterated and higher order multipoles considered.

resonance frequency $\Delta \omega_{h}=\omega_{h}-\omega_{h}^{\perp}$, and the corresponding shift in the resonance size parameter, i.e., $\Delta x_{h}=x_{h}-x_{h}^{\perp}$, are

$$
\frac{\Delta \omega_{h}}{\omega_{h}^{\perp}}=\frac{\Delta x_{h}}{x_{h}^{\perp}} \approx \frac{\kappa_{h}^{(2)}}{\operatorname{Re}\{\chi\}}=\frac{\kappa_{h}^{(2)}}{\kappa_{h}^{\perp}}\left(x_{h}^{\perp}\right)^{2}, \quad \operatorname{Re}\{\chi\} \gg 1 .
$$

In conclusion, the relative frequency shift of any dielectric mode is a quadratic function of $x_{h}^{\perp}$, whose prefactor is approximately the ratio between the second-order correction $\kappa_{h}^{(2)}$ and the quasistatic eigenvalue $\kappa_{h}^{\perp}$.
The radiative $\mathrm{Q}$-factor $\mathrm{Q}_{h}^{\perp \mathrm{r}}$ of the $h$ th dielectric mode is obtained as the inverse of the fractional bandwidth of the $h$ th addend of the second summation in Eq. (21), assuming negligible nonradiative losses $\operatorname{Im}\{\chi\} \approx 0$ and using the expansion (67):

$$
\mathrm{Q}_{h}^{\perp \mathrm{r}} \approx\left|\frac{\kappa_{h}^{\perp}}{\kappa_{h}^{\left(n_{i}\right)}}\right|\left(\frac{1}{x_{h}}\right)^{n_{i}} .
$$

The radiative Q-factor is an inverse power function of the size parameter whose exponent is the order $n_{i}$ of the first 
(a)

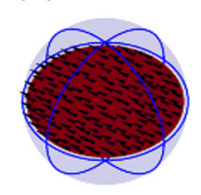

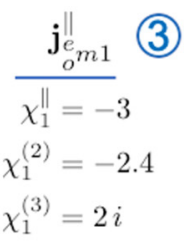

(b)

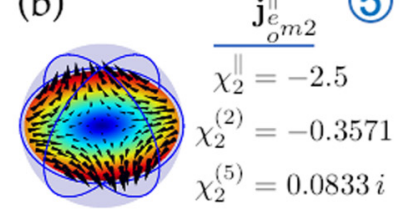

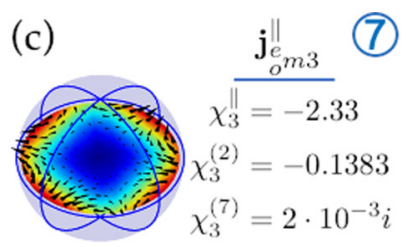

(d)

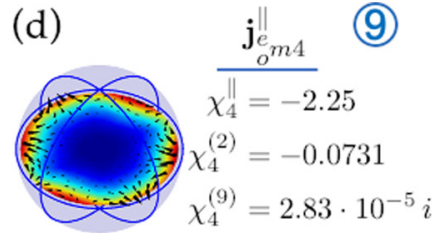

FIG. 2. Catalog of plasmonic resonances of a sphere. The electroquasistatic current density modes are ordered according to their eigenvalues $\chi_{n}^{\|}$. The second-order correction $\chi_{n}^{(2)}$ and the nonvanishing imaginary correction $\chi_{n}^{\left(n_{i}\right)}$ of lowest order $n_{i}$ are shown on the right of the corresponding panel, while the value of $n_{i}$ is shown on the top right enclosed in a circle.

nonvanishing imaginary correction $\kappa_{h}^{\left(n_{i}\right)}$, while the prefactor is the ratio between the quasistatic eigenvalue $\kappa_{h}^{\perp}$ and $\kappa_{h}^{\left(n_{i}\right)}$.

In Fig. 1, the algorithm for the calculation of the radiative Q-factor of any dielectric mode is summarized by a flowchart. First, the MQS current modes and corresponding eigenvalues are computed by solving the eigenvalue problem (8). Thus, if the mode exhibits a nonvanishing magnetic dipole moment, $\mathrm{Q}_{h}^{\perp \mathrm{r}}$ has the following expression, obtained by combining Eqs. (70) and (48):

$$
\mathrm{Q}_{h}^{\perp \mathrm{r}} \approx \frac{6 \pi}{\kappa_{h}^{\perp}\left\|\mathbf{P}_{\mathrm{M}|h|}^{\perp}\right\|^{2}}\left(\frac{1}{x_{h}}\right)^{3} .
$$

By expressing, by Eq. (13), the MQS eigenvalue $\kappa_{h}^{\perp}$ in terms of the magnetostatic energy $\mathscr{W}_{m}\left\{\mathbf{j}_{h}^{\perp}\right\}$ of the mode $\mathbf{j}_{h}^{\perp}, \mathrm{Q}_{h}^{\perp \mathrm{r}}$ is also found equal to $2 \pi$ times the ratio of $\mathscr{W}_{m}\left\{\mathbf{j}_{h}^{\perp}\right\}$ to the energy radiated to infinity in a period by the magnetic dipole $\mathbf{P}_{\mathrm{M} \mid h}^{\perp}$ at the resonance frequency $\omega_{h}$.

Instead, if the magnetic dipole vanishes, but at least one among the magnetic quadrupole moment $\overleftrightarrow{\mathbf{Q}}_{\mathrm{M}|h| i j}^{\perp}$, and the effective dipole moment resulting from the difference of the toroidal dipole moment $\mathbf{P}_{\mathrm{E} 2 \mid h}^{\perp}$ and the dipole moment of the second-order mode correction $\mathbf{P}_{\mathrm{E} \mid h}^{(2)}$ is nonvanishing, the radiative Q-factor has the following expression:

$$
\mathrm{Q}_{h}^{\perp \mathrm{r}} \approx \frac{1}{\kappa_{h}^{\perp}\left[\frac{1}{80 \pi} \sum_{i j}\left(Q_{\mathrm{M}|h| i j}^{\perp}\right)^{2}+\frac{1}{6 \pi}\left\|\mathbf{P}_{\mathrm{E} 2 \mid h}^{\perp}-\mathbf{P}_{\mathrm{E} \mid h}^{(2)}\right\|^{2}\right]}\left(\frac{1}{x_{h}}\right)^{5} .
$$

The above Q-factor is also equal to $2 \pi$ times the ratio of the magnetostatic energy of the $h$ th MQS mode to the sum of the energies radiated to infinity in a period by the magnetic quadrupole $\overleftrightarrow{\mathbf{Q}}_{\mathrm{M} \mid h}^{\perp}$ and by the effective dipole moment $\mathbf{P}_{\mathrm{E} 2 \mid h}^{\perp}-\mathbf{P}_{\mathrm{E} \mid h}^{(2)}$, at the resonance frequency $\omega_{h}$.
If they are all vanishing, the outlined algorithm has to be iterated, and higher order multipoles have to be considered.

In dielectric resonators, the opposite regime, dominated by nonradiative losses, is less common. Nevertheless, it is now considered for completeness. In this case, the nonradiative $\mathrm{Q}$-factor $\mathrm{Q}_{h}^{\perp \mathrm{nr}}$ is obtained as the inverse of the factional bandwidth of the $h$ th addend of the second summation in Eq. (21), assuming dominating nonradiative losses:

$$
\mathrm{Q}_{h}^{\perp \mathrm{nr}}=\frac{\left(\kappa_{h}^{\perp}\right)^{2}}{\operatorname{Im}\{\chi\}}\left(\frac{1}{x_{h}}\right)^{2} \approx \frac{\operatorname{Re}\{\chi\}}{\operatorname{Im}\{\chi\}},
$$

In an intermediate regime, the Q-factor, indicated with $\mathrm{Q}_{h}^{\perp}$, is obtained as [65]

$$
\frac{1}{\mathrm{Q}_{h}^{\perp}}=\frac{1}{\mathrm{Q}_{h}^{\perp \mathrm{r}}}+\frac{1}{\mathrm{Q}_{h}^{\perp \mathrm{nr}}}
$$

\section{RESULTS AND DISCUSSION}

Once the shape of an homogeneous object is assigned, its catalogs of plasmonic and dielectric modes can be introduced. The two catalogs are shown in Figs. 2 and 3 for a sphere, in Figs. 6 and 7 for a finite-size cylinder, and in Figs. 10 and 11 for a triangular prism. The catalogs illustrate the EQS and MQS current modes, where each set is ordered according to their real quasistatic eigenvalues $\chi_{h}^{\|}$and $\kappa_{h}^{\perp}$, respectively. Besides the quasistatic eigenvalue, each resonance is also characterized by the second-order corrections $\kappa_{h}^{(2)}$ and $\chi_{h}^{(2)}$, and the lowest-order (nonvanishing) imaginary corrections $\kappa_{h}^{\left(n_{i}\right)}, \chi_{h}^{\left(n_{i}\right)}$, where $n_{i}$ is odd with $n_{i} \geqslant 3$. In both tables, the value of $n_{i}$ is also highlighted, enclosed in a circle on the top right of each panel. Dielectric modes are labeled with " $\mathbb{A}^{\perp}$ " if they generate a transverse vector potential, accordingly to Definition (15). The information contained in these two cata$\operatorname{logs}$ depends neither on the permittivity of the object nor on
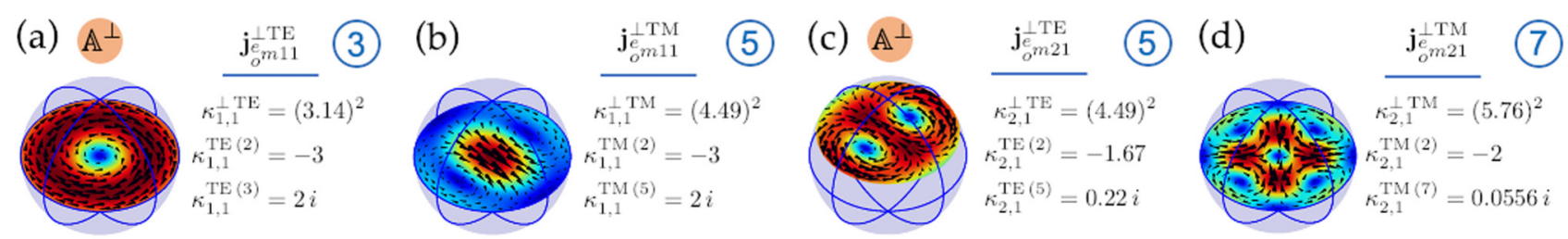

FIG. 3. Catalog of dielectric resonances of a sphere. The magnetoquasistatic current density modes are ordered according to their eigenvalue. The second-order correction and the nonvanishing imaginary correction of lowest order $n_{i}$ are shown on the right of the corresponding panel, while the value of $n_{i}$ of each mode is shown on the top right enclosed in a circle. The current modes generating a transverse vector potential are labeled with " $\mathbb{A}^{\perp}$." 
its size nor on the frequency of operation; it only depends on the morphology of the object.

The catalogs of plasmonic and dielectric resonances contain essential information to characterize and engineer the electromagnetic scattering of small objects. Specifically, the relative frequency shift of both plasmonic and dielectric resonances is a quadratic function of the size parameter at the quasistatic resonance, whose prefactor is $-\chi_{h}^{(2)} /\left(2 \chi_{h}^{\|}\right)$for plasmonic modes and $\kappa_{h}^{(2)} / \kappa_{h}^{\perp}$ for dielectric resonances, respectively. Furthermore, the radiative Q-factor is an inverse power function of the size parameter at the resonance, whose exponent is exactly the order $n_{i}$, while the prefactor is the ratio $\chi_{h}^{\|} / \chi_{h}^{\left(n_{i}\right)}$ for plasmonic and $\kappa_{h}^{\perp} / \kappa_{h}^{\left(n_{i}\right)}$ for dielectric modes. This prefactor only depends on the quasistatic eigenvalue and on the multipolar components of the quasistatic mode.

In this paper, the electrostatic eigenvalue problem (1) is solved by the surface integral method outlined in Refs. [1,51] using a triangular mesh. The magnetoquasistatic eigenvalue problem (8) is solved by the numerical method presented in Ref. [15] by using loop basis functions defined on a hexahedral mesh. Then, the radiation correction for both plasmonic [Eqs. (29), (33), and (34)] and dielectric eigenvalues [Eqs. (41), (48), and (51) are computed using standard quadrature formulas, and, if singular, using the formulas provided by Graglia [66,67].

\section{A. Sphere}

The plasmonic and dielectric resonances of a sphere of radius $R$ are now investigated. The characteristic length $l_{c}$ is assumed equal to the radius $R$. The sphere is the ideal shape to numerically validate the radiation corrections, because the quasistatic modes, the corresponding eigenvalues, and radiation corrections have analytic expressions, which are given in Appendix B. In the current section, these same quantities are calculated numerically and compared to their analytic counterparts. In particular, the surface mesh used for the calculation of EQS modes has 1500 nodes and 2996 triangles, while the hexahedral mesh used for the calculation of MQS modes has 6527 nodes, 6048 hexahedra, and 11665 edges. The same two meshes are also used for the computation of the surface and volume integrals of the radiation corrections. In Appendix F, the radiation correction of Eqs. (29), (33) and of (41), (48) are analytically calculated in few scenarios.

\section{Catalog of plasmonic resonances}

The catalog of plasmonic resonances of a sphere is shown in Fig. 2. The radiation corrections are calculated numerically and analytically, and the numerical error is shown in Table I. The three degenerate EQS modes $\mathbf{j}_{e_{m}}^{\|}$with $m=0,1$ (namely $\mathbf{j}_{e 01}^{\|}, \mathbf{j}_{e 11}^{\|}$, and $\mathbf{j}_{o 11}^{\|}$) are associated to the lowest eigenvalue $\chi_{1}^{\|}=-3$. The analytic expression of the modes is given in Eq. (B2) of Appendix B, while one of them is depicted in Fig. 2(a). They are bright and represent three electric dipoles oriented along mutually orthogonal directions. Their secondorder correction $\chi_{1}^{(2)}=-2.4$ is calculated both numerically, performing the integrals in Eq. (29), and analytically by
TABLE I. EQS eigenvalues of a sphere and their radiative corrections, obtained numerically by Eqs. (29), (33), and (34) and analytically by Eq. (B1). Relative error.

\begin{tabular}{lccc}
\hline \hline$\#$ & & $\mathbf{j}_{e^{\|}}$ & $\mathbf{j}_{o}^{\|}$ \\
\hline$\chi_{n}^{\|}$ & & -3.00 & $o_{o}{ }^{m 2}$ \\
& Numeric & -3 & -2.50 \\
& Analytic & 0.12 & -2.5 \\
& Error [\%] & -2.38 & 0.2 \\
\hline$\chi_{n}^{(2)}$ & Numeric & -2.4 & -0.350 \\
& Analytic & 1.1 & -0.357 \\
& Error [\%] & 1.98 & 3.0 \\
\hline$\chi_{n}^{(3)}$ & Numeric & 2 & $\approx 10^{-9}$ \\
& Analytic & 1.17 & 0 \\
& Error [\%] & & 0.0833 \\
\hline$\chi_{n}^{(5)}$ & Numeric & & 0.0816 \\
& Analytic & & 2.1 \\
\hline \hline
\end{tabular}

using Eq. (B1b). The third-order correction $\chi_{1}^{(3)}=+2 i$ is proportional to the squared magnitude of their electric dipole moment, and it is calculated both numerically by Eq. (33) and analytically by Eq. (B1c).

The next five degenerate EQS modes are $\mathbf{j}_{e_{m 2}}^{\|}$with $m=$ $0,1,3$. They are dark and exhibit a nonvanishing electric quadrupole tensor. These modes are exemplified in Fig. 2(b). The second-order correction is $\chi_{2}^{(2)}=-0.35$. The third-order correction $\chi_{2}^{(3)}$ vanishes since these modes are dark. The imaginary correction of the lowest order is $\chi_{2}^{(5)}=0.0833 i$, given by Eq. (34) and analytically by Eq. (B1c). It only depends on the electric quadrupole tensor of the EQS mode.

A similar line of reasoning can be also applied to octupolar modes $\mathbf{j}_{e^{m 3}}^{\|}$shown in Fig. 2(c) and to the hexadecapolar modes $\mathbf{j}_{e_{m}}^{\|}$shown in Fig. 2(d).

\section{Catalog of dielectric resonances}

The catalog of dielectric resonances of a sphere is shown in Fig. 3. The radiation corrections are calculated numerically and analytically, and the numerical error is shown in Table II. The MQS current modes $\mathbf{j}_{e_{m}}^{\perp \mathrm{TE}}$ with $m=0,1$ (namely $\mathbf{j}_{e 011}^{\perp \mathrm{TE}}$, $\mathbf{j}_{e 111}^{\perp \mathrm{TE}}$, and $\mathbf{j}_{o 111}^{\perp \mathrm{TE}}$ ) are associated to the lowest MQS eigenvalue, i.e., $\kappa_{11}^{\perp \mathrm{TE}}$. The analytic expressions of the modes and of the corresponding MQS eigenvalues are given in Eqs. (B6) and (B5a) of Appendix B. The current modes $\mathbf{j}_{e_{m 11}^{\perp T E}}^{\perp \mathrm{Tre}}$ three degenerate magnetic dipoles oriented along three orthogonal axis; one of them is shown in Fig. 3(a). They are $\mathbb{A}^{\perp}$ modes because they generate a transverse vector potential, namely with zero normal component to $\partial \tilde{\Omega}$, according to the definition given in Sec. II B. Thus, the second-order correction $\kappa_{11}^{\text {TE(2) }}$ has the simplified expression (43), because the magnetostatic interaction energy between $\mathbf{j}_{e_{m}}^{\perp \mathrm{TE}}$ and any EQS current 
TABLE II. MQS eigenvalues of a sphere and their radiative corrections, obtained numerically by Eqs. (41), (48), and (51) and analytically by Eqs. (B5) and (B9). Relative error.

\begin{tabular}{|c|c|c|c|c|}
\hline & & $\mathbf{j}_{e_{m}}^{\perp \mathrm{TE}}$ & $\mathbf{j}_{e_{m}{ }^{\perp \mathrm{TM}}}^{\perp \mathrm{M}}$ & $\mathbf{j}_{e_{m}}^{\perp \mathrm{TE}}$ \\
\hline \multirow[t]{3}{*}{$\sqrt{\kappa_{n l}^{\perp}}$} & Numeric & 3.16 & 4.53 & 4.52 \\
\hline & Analytic & 3.14 & 4.49 & 4.49 \\
\hline & Error [\%] & 0.64 & 1.81 & 1.04 \\
\hline \multirow[t]{3}{*}{$\kappa_{n l}^{(2)}$} & Numeric & -3.02 & -3.08 & -1.69 \\
\hline & Analytic & -3 & -3 & -1.67 \\
\hline & Error $[\%]$ & 0.67 & 2.3 & 1.2 \\
\hline \multirow[t]{3}{*}{$\kappa_{n l}^{(3)}$} & Numeric & 2.0 & 0 & 0 \\
\hline & Analytic & 2 & 0 & 0 \\
\hline & Error [\%] & 0.14 & & \\
\hline \multirow{3}{*}{$\kappa_{n l}^{(5)}$} & Numeric & & 2.1 & 0.22 \\
\hline & Analytic & & 2 & 0.22 \\
\hline & Error [\%] & & 4.9 & 0.7 \\
\hline
\end{tabular}

mode vanishes, i.e., $\mathscr{W}_{m \mathrm{I}}\left\{\mathbf{j}_{e_{o^{\prime} n^{\prime}}}^{\|}, \mathbf{j}_{e_{m}}^{\perp \mathrm{TE}}\right\}=0 \forall m^{\prime}, n^{\prime} \geqslant m^{\prime}$. The second-order correction also feature the analytic expression (B5b). The third-order correction $\kappa_{11}^{\mathrm{TE}(3)}$ is given by Eq. (48) and is proportional to the squared magnitude of the magnetic dipole moment of the mode; its analytic expression is given in Eq. (B5c).

The next three degenerate modes, namely $\mathbf{j}_{e_{m}}^{\perp \mathrm{TM}}$ with $m=$ 0, 1, are shown in Fig. 3(b). The modes analytic expression is given in Eq. (B10). Each of them generates a vector potential with a nonvanishing longitudinal component. Specifically, the magnetostatic interaction energies between the current modes $\mathbf{j}_{e^{m 11}}^{\perp \mathrm{TM}}$ and the EQS modes $\mathbf{j}_{e_{m^{\prime} 1}^{\|}}^{\|}$with $m^{\prime}=0,1$ [shown in Fig. 2(a)] are nonvanishing. They contribute to the secondorder correction $\kappa_{11}^{\mathrm{TM}(2)}$, as prescribed by Eq. (41). The second-order correction has also the analytic expression given in Eq. (B9b). The third-order correction $\kappa_{11}^{\mathrm{TM}(3)}$ vanishes because the magnetic dipole moment of these modes is zero. Thus, the first nonvanishing imaginary correction is $\kappa_{11}^{\mathrm{TM}(5)}$, given by Eq. (51) and analytically by Eq. (B9c). Since the modes $\mathbf{j}_{e_{m}}^{\perp \mathrm{TM}}$ have a vanishing magnetic quadrupole tensor, the correction $\kappa_{11}^{\mathrm{TM}(5)}$ originates only from an effective dipole moment resulting from the interplay between the dipole moment of the second-order mode correction and the toroidal dipole.

The next five degenerate modes are $\mathbf{j}_{e_{m 21}}^{\perp \mathrm{TE}}$ with $m=0,1,2$ and are shown in Fig. 3(c). As already pointed out in Ref. [15], the modes $\mathbf{j}_{e_{m} 1}^{\perp \mathrm{TE}}$ have the same MQS eigenvalue of $\mathbf{j}_{e_{e_{m}}}^{\perp \mathrm{TM}}$. Nevertheless, unlike them, they are $\mathbb{A}^{\perp}$ modes. For this reason, the second-order correction $\kappa_{21}^{\mathrm{TE}(2)}$ has the simplified expression (43). Their magnetic dipole moment is zero, and thus the third-order correction vanishes. The toroidal dipole moment is zero as well as the electric dipole moment of the secondorder mode correction. Nevertheless, the magnetic quadrupole

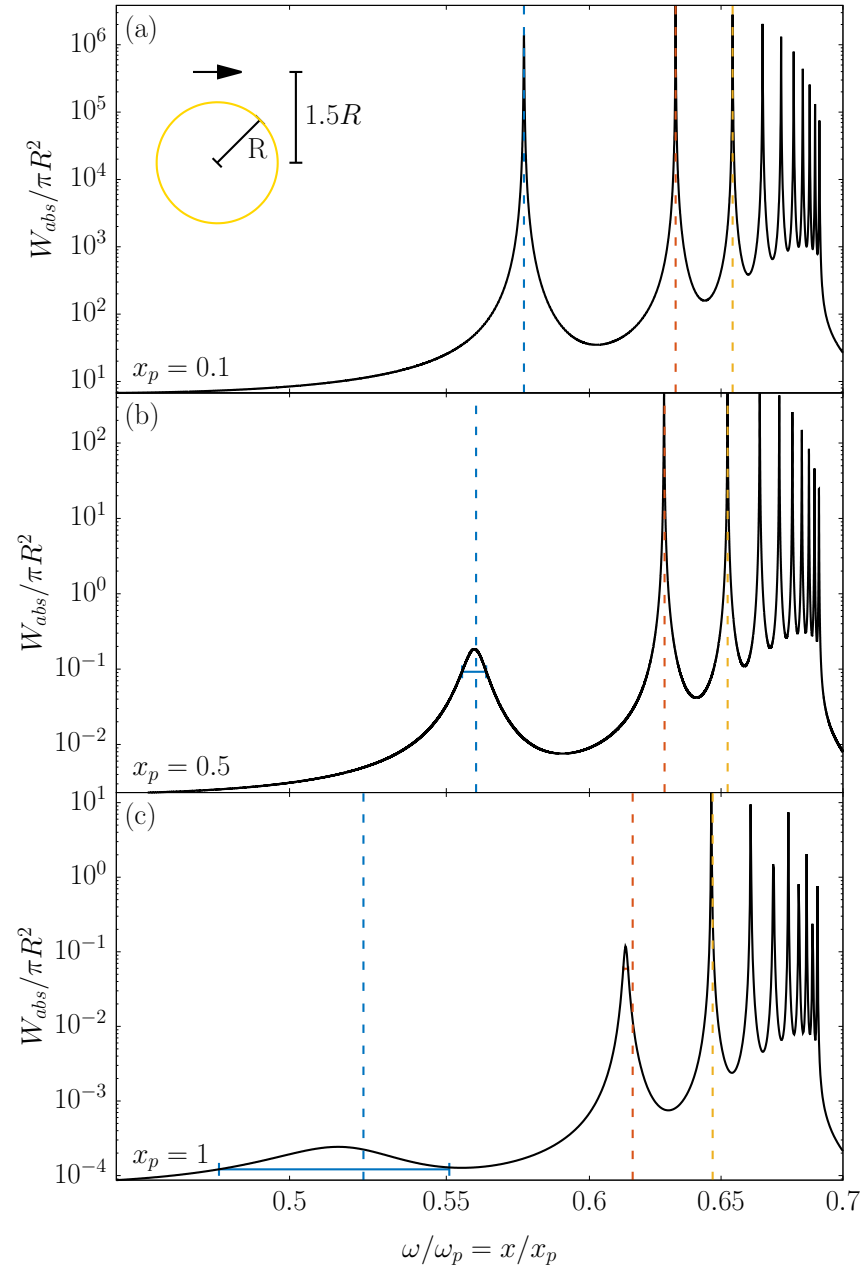

FIG. 4. Power $W_{a b s}$ absorbed by a Drude metal sphere ( $v=$ $10^{-4} \omega_{p}$ ) with radius $R=l_{c}$ as a function of $\omega / \omega_{p}=x / x_{p}$ for $x_{p}=$ 0.1 (a), $x_{p}=0.5$ (b), and $x_{p}=0.5$ (c). The sphere is centered in $(0,0,0)$ and it is excited by a point source oriented along $\hat{\mathbf{x}}$, i.e., $\mathbf{E}_{i n c}(\mathbf{r})=\mathbf{N}_{e 11}^{(3)}$, at position $(0,0,1.5 R)$, as in the inset. The resonance frequencies obtained by Eq. (58) are shown with vertical dashed lines. Horizontal lines show the FWHM of the broadest peaks.

tensor is nonvanishing and the fifth-order correction $\kappa_{21}^{\mathrm{TE}(5)}$ can be calculated by Eq. (52).

The outlined method can be iterated to describe the degenerate modes $\mathbf{j}_{e_{m}}^{\perp \mathrm{TM}}$ with $m=0,1,2$, shown in Fig. 3(d), which involve higher order multipoles.

\section{Point source excitation}

The sphere is now excited by a point source. Specifically, the sphere of radius $R$ is centered in the origin, while the point source is oriented along $\hat{\mathbf{x}}$ and it is positioned at $\mathbf{r}_{d}=$ $(0,0,1.5 R)$, namely

$$
\mathbf{E}_{i n c}(\mathbf{r})=\mathbf{N}_{e 11}^{(3)}\left(\mathbf{r}-\mathbf{r}_{d}\right),
$$

where $\mathbf{N}_{e 11}^{(3)}$ is a vector spherical wave function of the radiative kind, defined in Eq. (C1) of Appendix $\mathrm{C}$ following Ref. [68]. Under the same excitation conditions, two different scenarios are investigated. In the first one, shown in Fig. 4, 


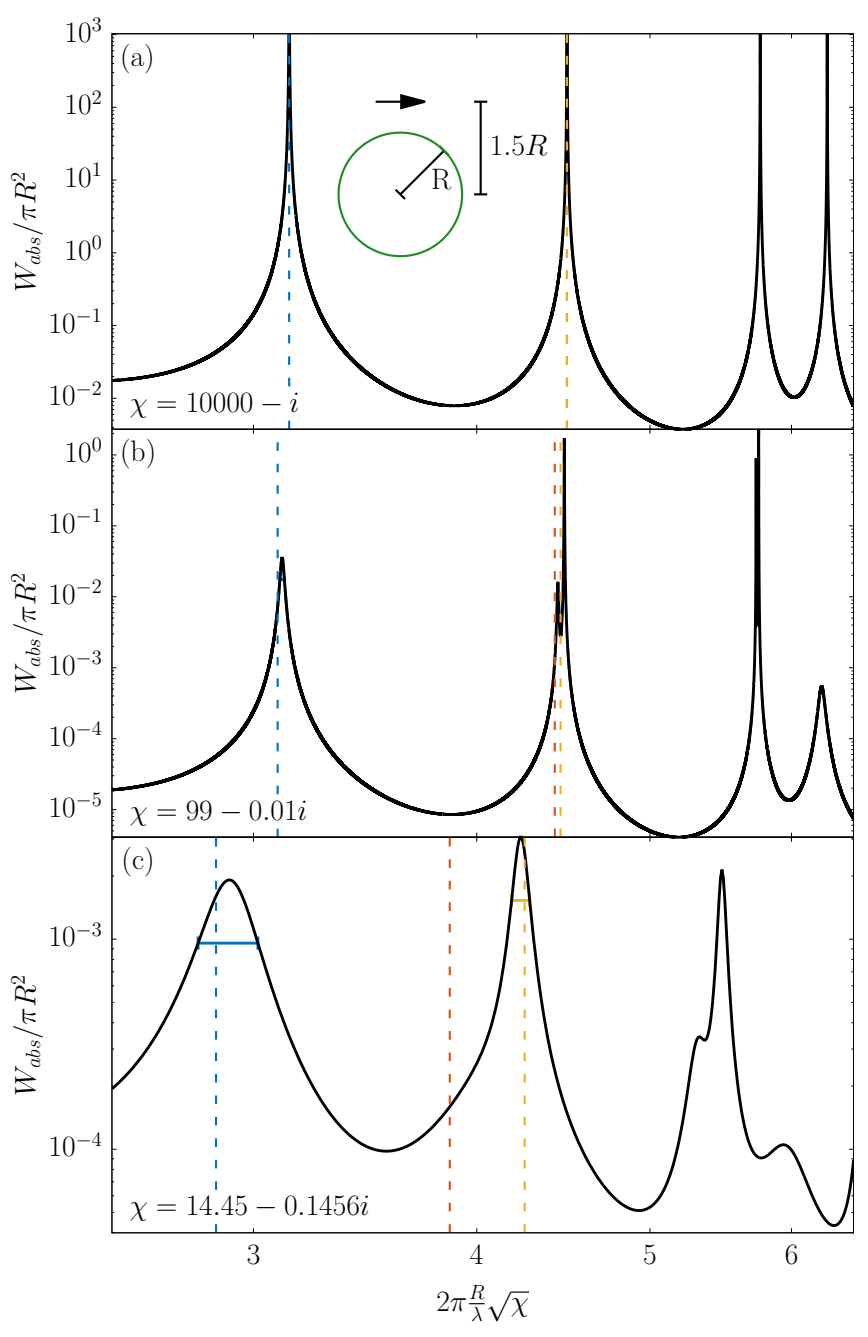

FIG. 5. Power $W_{a b s}$ absorbed by a dielectric sphere with radius $R=l_{c}$, and susceptibility $\chi=$ (a) $10^{4}-1 i$, (b) $99-0.01 i$, and (c) $14.45-0.1456 i$, as a function of $x \sqrt{\chi}$. The sphere is centered in $(0,0,0)$ and it is excited by point source oriented along $\hat{\mathbf{x}}$, e.g., $\mathbf{E}_{\text {inc }}(\mathbf{r})=\mathbf{N}_{e 11}^{(3)}$ at position $(0,0,1.5 R)$, as in the inset. The first three resonance positions obtained by Eq. (68) are shown with vertical dashed lines. Horizontal lines show the FWHM of the broadest peaks.

the sphere is made of a Drude metal; in the second one, shown in Fig. 5, it is made of a high-index dielectric. In both cases, low losses are assumed: This hypothesis is essential for a quantitative comparison between the predicted radiative Q-factor and fractional bandwidth of the peaks, which would have been otherwise often dominated by nonradiative losses. The absorbed power spectrum $W_{a b s}$ is chosen as physical observable. It is normalized by the geometrical cross section $\pi l_{c}^{2}$, and features the expression

$$
\frac{W_{a b s}}{\pi l_{c}^{2}}=\frac{x}{2 \pi \zeta_{0}}\left(-\operatorname{Im}\left\{\varepsilon_{R}\right\}\right) \int_{\tilde{\Omega}}\|\mathbf{E}(\tilde{\mathbf{r}})\|^{2} d \tilde{V},
$$

where $\mathbf{E}$ is the total electric field within the particle and $\zeta_{0}=\sqrt{\mu_{0} / \varepsilon_{0}}$ is the vacuum characteristic impedance. In this section, it is calculated by the Mie analytic solution [68] combined with the translation-addition theorem for vector spherical wave functions (VSWFs) $[69,70]$ that is used to translate the VSWF of the point source excitation into the corresponding VSWFs set centered in the sphere's center. The maximum multipolar expansion order for the Mie solution is assumed to be 20 .

The reason behind the choice of the point source as excitation and of the absorbed power as physical observable is that without these two hypothesis, some modes may not be excited or probed.

The radiative shift of the peaks of $W_{a b s}$ and their Q-factors are investigated as the object size increases. In particular, the plasmonic and dielectric resonance frequencies are compared against the frequencies at which the curve has a peak, denoted as $\hat{\omega}_{h}$. Similarly, the Q-factors of plasmonic and dielectric modes are validated against the corresponding heuristic Qfactors, given by the ratio of the resonance frequency $\hat{\omega}_{h}$ to the width $\Delta \omega_{\mathrm{FWHM}, h}$ of the resonance curve between two points, at the either side of the resonance, where the ordinate is the half of the maximum absorbed power, namely the full width at half maximum (FWHM) [71],

$$
\hat{Q}_{h}=\frac{\hat{\omega}_{h}}{\Delta \omega_{\mathrm{FWHM}, h}} .
$$

In the $W_{a b s}$ spectra of Figs. 4 and 5, a segment joining the two ordinates at half maximum is also shown.

Metal sphere. A metal sphere is investigated in Fig. 4, assuming a low-loss Drude metal with $v=10^{-4} \omega_{p}$. The absorbed power spectra $W_{a b s}$ are evaluated as a function of $x / x_{p}=\omega / \omega_{p}$ for three different values of $x_{p}: 0.1$ in Fig. 4(a), 0.5 in Fig. 4(b), and 1 in Fig. 4(c). It is useful to contextualize the chosen values of $x_{p}$ to actual materials: For a gold sphere [72] with $\omega_{p} \approx 6.79 \mathrm{~T} \mathrm{rad} / \mathrm{s}$, they correspond to $R=4.5 \mathrm{~nm}$ [Fig. 4(a)], $R=22 \mathrm{~nm}$ [Fig. 4(b)], and $R=45 \mathrm{~nm}$ [Fig. 4(c)]. The resonance positions of the first three excited plasmonic modes, which in the quasistatic limit tend to the EQS modes $\mathbf{j}_{e 11}^{\|}$(electric dipole), $\mathbf{j}_{e 12}^{\|}$(electric quadrupole), and $\mathbf{j}_{e 13}^{\|}$(electric octupole), are obtained by Eq. (58) and are shown with vertical dashed lines (blue, red, yellow, respectively).

In the small particle limit, accordingly to Eq. (59), the relative frequency shift of any resonance (with respect to its quasistatic position) is a quadratic function of the size parameter at the EQS resonance, whose prefactor depends on the ratio between the second-order correction $\chi_{n}^{(2)}$ and the EQS eigenvalue $\chi_{n}^{\|}$. Using the value of corrections shown in Fig. 2, this ratio is found to be significantly larger for $\mathbf{j}_{e 11}^{\|}$than for $\mathbf{j}_{e 12}^{\|}$ and $\mathbf{j}_{e 13}^{\|}$, and thus the dipole mode $\mathbf{j}_{e 11}^{\|}$is expected to exhibit the largest frequency shift.

Moreover, the order $n_{i}$ of the first nonvanishing imaginary correction is 3 for the electric dipole mode $\mathbf{j}_{e 11}^{\|}, 5$ for the electric quadrupole mode $\mathbf{j}_{e 12}^{\|}$, and 7 for electric octupole mode $\mathbf{j}_{e 13}^{\|}$; thus, the dipole mode is also expected to undergo the largest radiative broadening.

In Fig. 4(a), $x_{p}=0.1$ and the radius $R$ is small compared to the plasma wavelength $\lambda_{p}$, and thus the EQS approximation works well: Eq. (55) exactly predicts the occurrence of the $W_{a b s}$ peaks.

In Fig. 4(b), $x_{p}$ is increased to 0.5 , and the $W_{a b s}$ peaks undergo a broadening and shift from their quasistatic position, in particular the peak associated to the electric dipole 
TABLE III. Resonance frequencies $\omega_{n}$ and Q-factors $\mathrm{Q}_{n}^{\| \mathrm{r}}, \mathrm{Q}_{n}^{\| \mathrm{nr}}$, $\mathrm{Q}_{n}^{\|}$of the first plasmonic modes of a Drude metal sphere $(v=$ $\left.10^{-4} \omega_{p}\right)$, and their heuristic estimates $\hat{\omega}_{n}$ and $\hat{\mathrm{Q}}_{n}$.

\begin{tabular}{ccccc}
\hline \hline$x_{p}$ & & $\mathbf{j}_{e 11}^{\|}$ & $\mathbf{j}_{e 12}^{\|}$ \\
\hline 0.5 & Theory & $\omega_{n} / \omega_{p}$ & 0.560 & 0.628 \\
& Heuristic & $\hat{\omega}_{n} / \omega_{p}$ & 0.560 & 0.632 \\
& Theory & $\mathrm{Q}_{n}^{\| \mathrm{r}}$ & 68.3 & 1010 \\
& $\mathrm{Q}_{n}^{\| n \mathrm{nr}}$ & 5660 & 6280 \\
& $\mathrm{Q}_{n}^{\|}$ & 67.5 & 3813 \\
& & $\hat{\mathrm{Q}}_{n}$ & 66.6 & 3875 \\
\hline 1 & Heuristic & $\omega_{n} / \omega_{p}$ & 0.523 & 0.616 \\
& Theory & $\hat{\omega}_{n} / \omega_{p}$ & 0.515 & 0.6133 \\
& Heuristic & $\mathrm{Q}_{n}^{\| \mathrm{r}}$ & 10.5 & 340 \\
& Theory & $\mathrm{Q}_{n}^{\| n \mathrm{nr}}$ & 5229 & 6159 \\
& & $\mathrm{Q}_{n}^{\|}$ & 10.5 & 320 \\
& & $\hat{\mathrm{Q}}_{n}$ & 7.15 & 340 \\
\hline \hline
\end{tabular}

$\mathbf{j}_{e 11}^{\|}$. Nevertheless, the resonance positions obtained through Eq. (58), which incorporates the radiation corrections, accurately predict the occurrence of the $W_{a b s}$ peaks. In Table III, the resonance frequencies $\omega_{n}$ are compared against the corresponding peak positions $\hat{\omega}_{n}$, and the Q-factors are compared against their heuristic counterparts. In particular, the radiative and nonradiative Q-factors are calculated by Eqs. (60) and (63) and combined to obtain the total Q-factor by Eq. (64). Analytic expressions for the radiative Q-factors of the EQS modes of a sphere are also derived in (B3), which agree with the ones provided in Ref. [73]. As expected, for $x_{p}=0.5$ the $\mathrm{Q}$-factor of the dipole mode is the lowest one, and it is limited by radiative losses, unlike all the others.

Eventually, in Fig. 4(c) $x_{p}=1$, and thus $R$ is comparable to the plasma wavelength $\lambda_{p}$. The peaks experience a further shift; nevertheless, thanks to the radiation corrections, Eq. (58) is still able to accurately locate the resonances with an error $<1.5 \%$, as shown in Table III. Also, the Q-factors are predicted with good accuracy. The Q-factor of the dipole and the quadrupole modes are now both dominated by radiative losses.

High-index sphere. In Fig. 5, the power absorbed by a sphere made of a nondispersive high-index dielectric with low losses is investigated as a function of the parameter $x \sqrt{\chi}$. Three different values of susceptibility $\chi$ are considered, namely $\chi=10^{4}-1 i, \chi=99-0.01 i$, and $\chi=$ $14.45-0.1456 i$.
TABLE IV. Resonance position $x_{h} \sqrt{\chi}$ and Q-factors $\mathrm{Q}_{h}^{\perp \mathrm{r}}, \mathrm{Q}_{h}^{\perp \mathrm{nr}}$, $\mathrm{Q}_{h}^{\perp}$ of the first dielectric modes of a dielectric sphere with different values susceptibility $\chi$, and their heuristic estimates $\hat{x}_{h} \sqrt{\chi}$ and $\hat{Q}_{h}$.

\begin{tabular}{lccccc}
\hline \hline$\chi$ & & & $\mathbf{j}_{o 111}^{\perp \mathrm{TE}}$ & $\mathbf{j}_{e 111}^{\perp \mathrm{TM}}$ & $\mathbf{j}_{\text {o121 }}^{\perp \mathrm{TE}}$ \\
\hline $99-0.01 i$ & Theory & $x_{h} \sqrt{\chi}$ & 3.095 & 4.456 & 4.423 \\
& Heuristic & $\hat{x}_{h} \sqrt{\chi}$ & 3.113 & 4.477 & 4.441 \\
& Theory & $\mathrm{Q}_{h}^{\perp \mathrm{r}}$ & 163 & 579 & 5043 \\
& & $\mathrm{Q}_{h}^{\perp \mathrm{nr}}$ & $10^{4}$ & $10^{4}$ & $10^{4}$ \\
& $\mathrm{Q}_{h}^{\perp}$ & 163 & 547 & 3352 \\
& & $\hat{\mathrm{Q}}_{h}$ & 171 & 448 & 3731 \\
\hline \multirow{2}{*}{ Heuristic } & Theory & $x_{h} \sqrt{\chi}$ & 2.860 & 3.863 & 4.255 \\
& Heuristic & $\hat{x}_{h} \sqrt{\chi}$ & 2.907 & & 4.237 \\
& Theory & $\mathrm{Q}_{h}^{\perp} \approx \mathrm{Q}_{h}^{\perp \mathrm{r}}$ & 11.60 & 9.6 & 51.7 \\
& Heuristic & $\hat{\mathrm{Q}}_{h}$ & 13 & & 43 \\
\hline \hline
\end{tabular}

The resonance positions of the three dielectric modes which tend in the quasistatic limit to the MQS modes $\mathbf{j}_{o 111}^{\perp \mathrm{TE}}$ (magnetic dipole), $\mathbf{j}_{e 111}^{\perp \mathrm{TM}}$, and $\mathbf{j}_{o 121}^{\perp \mathrm{TE}}$ (magnetic quadrupole) are obtained by Eq. (68). They are highlighted in Fig. 5 by blue, red, and yellow vertical dashed lines, respectively. Accordingly to Eq. (69), the resonance frequency shift is a quadratic function of the size parameter at the MQS resonance, whose prefactor depends on the ratio between the second-order correction and the MQS eigenvalue. Using the corrections' values reported in Fig. 3, this ratio is found to be larger for the magnetic dipole $\mathbf{j}_{o 111}^{\perp \mathrm{TE}}$ than for $\mathbf{j}_{e 111}^{\perp \mathrm{TM}}$ or $\mathbf{j}_{e 111}^{\perp \mathrm{TM}}$, and thus this mode is expected to undergo the largest frequency shift.

In Fig. 5(a), it is assumed $\chi=10^{4}-i$ and $x \in$ $[0.025,0.065]$. The size parameter is very small and the MQS approximation works well: The predictions of Eqs. (65) and (68) agree and exactly predicts the occurrence of the $W_{a b s}$ peaks.

In Fig. 5(b), it is assumed $\chi=99-0.01 i$ and $x \in$ $[0.25,0.65]$. The peak position red shifts against the MQS position, but Eq. (68), which includes the radiation corrections, predicts the occurrence. In Table IV, the expected resonance frequencies and Q-factors are compared against the peak positions and the corresponding heuristic Q-factors. In particular, the radiative and nonradiative Q-factors are calculated by Eqs. (70) and (73) and combined by Eq. (74). Analytic expressions of the Q-factors of MQS modes of a sphere are also provided in Eqs. (B7) and (B12) of the Appendix. The agreement of the total Q-factors with their heuristic counterparts is good. The radiative damping determines the broadening of the
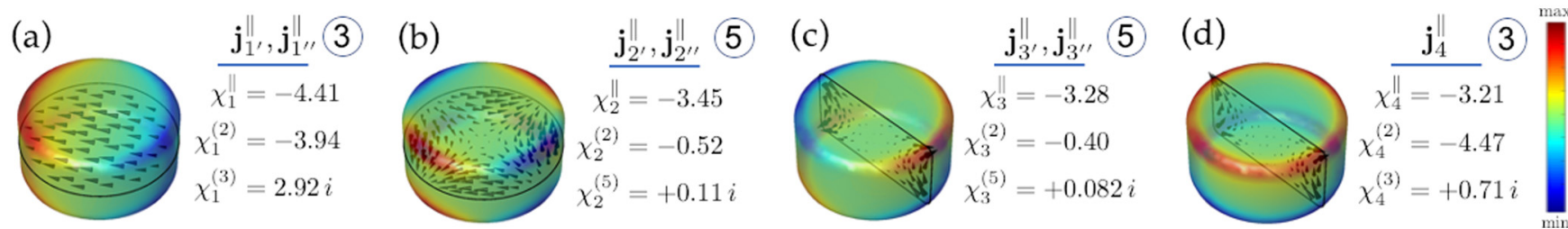

FIG. 6. Catalog of plasmonic resonances of a finite-size cylinder with $l_{c}=R=H$. The electroquasistatic current density modes $\mathbf{j}_{h}^{\|}$are ordered according to their eigenvalue $\chi_{h}^{\|}$. Their field lines are shown with black arrows on representative planes, and their normal component on $\partial \Omega$ is represented with colors. The second-order correction $\chi_{h}^{(2)}$, and the nonvanishing imaginary correction $\chi_{h}^{(n)}$ of lowest order $n_{i}$ are shown on the right of the corresponding panel, while the value of $n_{i}$ is also highlighted on the top right enclosed in a circle. 

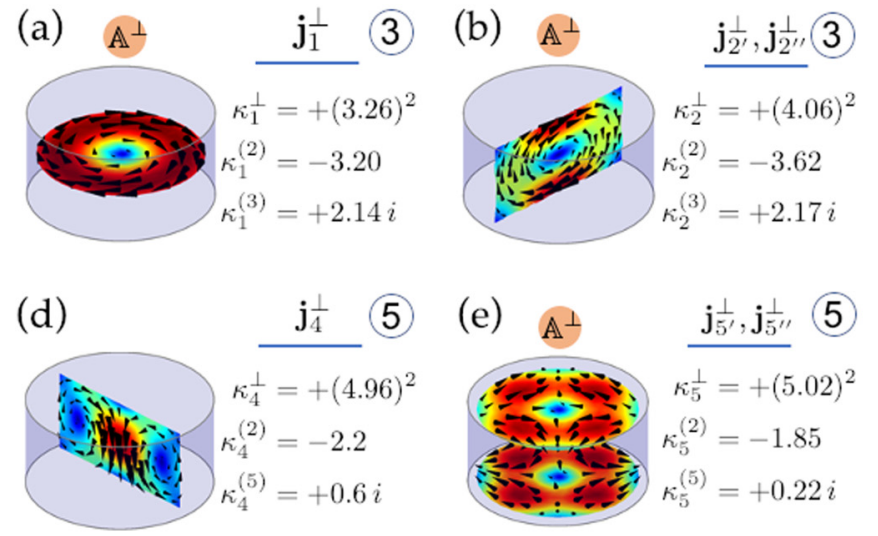
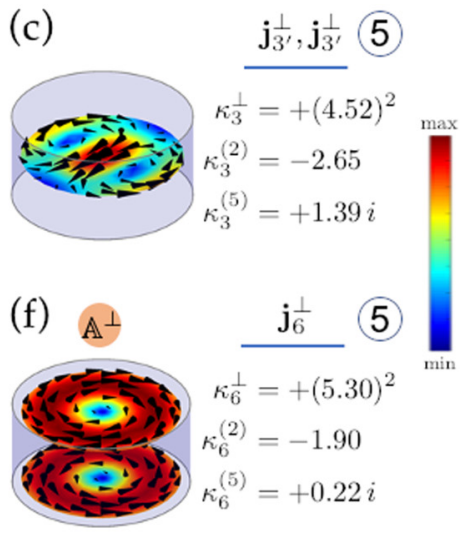

FIG. 7. Catalog of dielectric resonances of a finite-size cylinder with $l_{c}=R=H$. The magnetoquasistatic current density modes are ordered according to their eigenvalue $\kappa_{h}^{\perp}$. The second-order correction $\kappa_{h}^{(2)}$ and the nonvanishing imaginary correction $\kappa_{h}^{(n)}$ of lowest order $n_{i}$ are shown on the right of the corresponding panel, while the value of $n_{i}$ is also highlighted on the top right enclosed in a circle. The current modes generating a transverse vector potential are labeled with $\mathbb{A}^{\perp}$.

magnetic dipole modes, while nonradiative mechanisms play an important role for remaining peaks.

Eventually, in Fig. 5(c), a silicon sphere with $\chi=14.45-$ $0.1456 i$ is investigated in the range $x \in[0.64,1.6]$. The radius is now comparable to the incident wavelength. The shift of the peaks against the MQS position is significant, but including the radiation corrections, Eq. (68) predicts their occurrence with an error $\leqslant 2 \%$. As shown in Table IV, the predicted Q-factors are very close to their heuristic counterparts and dominated by radiative losses. The second peak is not visible anymore due to the radiative broadening, and its heuristic peak position and Q-factor cannot be evaluated.

\section{B. Finite-size cylinder}

A finite-size cylinder with radius $R$ and height $H=R$ is now considered. The edges are rounded with a curvature radius of $R / 10$. The characteristic length $l_{c}$ is assumed to be equal to the radius $R$. The triangular surface mesh used for the calculation of the EQS modes has 2063 nodes and 4122 triangles. The hexahedral mesh used for the calculation of MQS modes has 6060 nodes, 5148 hexahedra, and 9433 edges.

\section{Catalog of plasmonic resonances}

The catalog of the first four plasmonic resonances is shown in Fig. 6. The two degenerate EQS current modes $\left\{\mathbf{j}_{1^{\prime}}^{\|}, \mathbf{j}_{1^{\prime \prime}}^{\|}\right\}$ have the smallest EQS eigenvalue. They represent two electric dipoles oriented along mutually orthogonal directions, which are orthogonal to the cylinder's axis, and thus they are bright. The second-order correction $\chi_{1}^{(2)}=-3.94$ is obtained by Eq. (29). The third-order correction $\chi_{1}^{(3)}=+2.92 i$, given by Eq. (33), is proportional to the squared magnitude of the electric dipole moment of the EQS modes.

The next EQS modes are two degenerate couples, namely $\left\{\mathbf{j}_{2^{\prime}}^{\|}, \mathbf{j}_{2^{\prime \prime}}^{\|}\right\}$and $\left\{\mathbf{j}_{3^{\prime}}^{\|}, \mathbf{j}_{3^{\prime \prime}}^{\|}\right\}$, shown in Figs. 6(b) and 6(c). They are dark; thus, the third-order corrections $\chi_{2}^{(3)}$ and $\chi_{3}^{(3)}$ vanish. In both cases, the lowest order of the first nonvanishing imaginary correction is the fifth. Accordingly to Eq. (34), $\chi_{2}^{(5)}$ and $\chi_{3}^{(5)}$ depend on the electric quadrupole tensor of the corresponding EQS mode.
In Fig. 6(d), the EQS mode $\mathbf{j}_{4}^{\|}$is shown. This mode is associated to an electric dipole aligned along the cylinder's axis; thus, it is bright. The second-order correction is $\chi_{4}^{(2)}=-4.47$. The third-order correction is proportional to the squared magnitude of the electric dipole moment of the EQS current mode; its value is $\chi_{4}^{(3)}=+0.71 i$.

\section{Catalog of dielectric resonances}

The catalog of the first six dielectric resonances of the finite-size cylinder is presented in Fig. 7. The mode $\mathbf{j}_{1}^{\perp}$ is associated to the lowest MQS eigenvalue, shown in Fig. 7(a), which is a magnetic dipole oriented along the cylinder axis. The current mode $\mathbf{j}_{1}^{\perp}$ is an $\mathbb{A}^{\perp}$ mode, since it generates a vector potential with a longitudinal component of the vector potential which is numerically negligible. Thus, the magnetostatic interaction energy between the current mode $\mathbf{j}_{1}^{\perp}$ and any EQS current mode is zero, and the second-order correction $\kappa_{1}^{(2)}$ is simply given by Eq. (43). The third-order correction is proportional to the squared magnitude of the magnetic dipole moment of $\mathbf{j}_{1}^{\perp}$, accordingly to Eq. (48).

The successive couple of degenerate modes $\left\{\mathbf{j}_{2^{\prime}}^{\perp}, \mathbf{j}_{2^{\prime \prime}}^{\perp}\right\}$, shown in Fig. 7(b), are magnetic dipoles oriented along two mutually orthogonal directions, which are both orthogonal to the cylinder's axis. Analogously to $\mathbf{j}_{1}^{\perp}$, they are $\mathbb{A}^{\perp}$ modes, and similar considerations apply.

The next degenerate modes, namely $\left\{\mathbf{j}_{3^{\prime}}^{\perp}, \mathbf{j}_{3^{\prime \prime}}^{\perp}\right\}$, are shown in Fig. 7(c). They are known as $\mathrm{HEM}_{12 \delta}$ within the antenna community [9]. Unlike the previous modes, these current modes generate a vector potentials with a nonvanishing longitudinal component. Thus, accordingly to Eq. (41), the second-order correction $\kappa_{3}^{(2)}$ also depends on the interaction energy between the MQS current modes $\mathbf{j}_{3^{\prime}}^{\perp}$ and $\mathbf{j}_{3^{\prime \prime}}^{\perp}$ and the two horizontal EQS current modes $\mathbf{j}_{1^{\prime}}^{\|}, \mathbf{j}_{1^{\prime \prime}}^{\|}$, shown in Fig. 6(a). The coupling with the remaining EQS modes is instead negligible. Since the modes $\mathbf{j}_{3^{\prime}}^{\perp}$ and $\mathbf{j}_{3^{\prime \prime}}^{\perp}$ have zero magnetic dipole moment, the third-order correction vanishes. The imaginary correction of the lowest order is $\kappa_{3}^{(5)}$, and it is determined by the multipolar contribution from the toroidal dipole moment $\mathbf{P}_{\mathrm{E} 2 \mid 3}^{\perp}$ and from the electric dipole moment of the second-order correction $\mathbf{P}_{\mathrm{E} \mid 3}^{(2)}$. 
The next mode is $\mathbf{j}_{4}^{\perp}$, also known [9] as $\mathrm{TM}_{01 \delta}$, and it is shown in Fig. 7(d). It generates a vector potential with a nonvanishing longitudinal component. In particular, its magnetostatic interaction energy with the EQS mode $\mathbf{j}_{4}^{\|}$contributes to the second-order correction $\kappa_{4}^{(2)}$ of Eq. (41). The mode $\mathbf{j}_{4}^{\perp}$ has zero magnetic dipole moment, thus $\kappa_{4}^{(3)}=0$, while its fifth-order correction is nonvanishing and determined by the multipoles $\mathbf{P}_{\mathrm{E} 2 \mid 4}^{\perp}$ and $\mathbf{P}_{\mathrm{E} \mid 4}^{(2)}$.

The subsequent two sets of degenerate modes $\left\{\mathbf{j}_{5^{\prime}}^{\perp}, \mathbf{j}_{5^{\prime \prime}}^{\perp}\right\}$ and $\mathbf{j}_{6}^{\perp}$, shown in Figs. 7(e) and 7(f), are $\mathbb{A}^{\perp}$ modes. Therefore, in both cases, the second-order correction is given by Eq. (43). They exhibit zero magnetic dipole moment and thus vanishing third-order correction. The fifth-order corrections $\kappa_{5}^{(5)}$ and $\kappa_{6}^{(5)}$ can be calculated by Eq. (52) and originate from the magnetic quadrupoles $\overleftrightarrow{\mathbf{Q}}_{\mathrm{M} \mid 5}^{\perp}$ and $\overleftrightarrow{\mathbf{Q}}_{\mathrm{M} \mid 6}^{\perp}$ of the corresponding MQS modes.

\section{Point source excitation}

Now, the finite-size cylinder is excited by a point source. Specifically, the cylinder of radius $R$ and height $H=R$ is centered in the origin, its axis is oriented along $\hat{\mathbf{z}}$, while the point source is oriented along $\hat{\mathbf{y}}$ and it is positioned at $\mathbf{r}_{d}=(R, 0,1.5 R)$, namely

$$
\mathbf{E}_{i n c}(\mathbf{r})=\mathbf{N}_{o 11}^{(3)}\left(\mathbf{r}-\mathbf{r}_{d}\right) .
$$

Assuming the same geometry and excitation conditions, two different scenarios are investigated. In the first one, the cylinder is made of a Drude metal, and in the second, it is made by a high-index dielectric. The absorbed power spectrum is calculated by an in-house full-wave numerical method based on surface integral equations [74], using a triangular mesh with 1106 nodes and 2208 triangles. The frequency shift and the broadening of the resonances are analyzed.

Metal cylinder. In Fig. 8, the first scenario is investigated, assuming a low-loss Drude metal with $v=10^{-3} \omega_{p}$. The absorbed power $W_{a b s}$ is shown as a function of $x / x_{p}=\omega / \omega_{p}$ for three different values of $x_{p}$ : 0.1 in Fig. 8(a), 0.5 in Fig. 8(b), and 1 in Fig. 8(c). For gold cylinders, by assuming, as in Ref. [72], $\omega_{p} \approx 6.79 \mathrm{~T} \mathrm{rad} / \mathrm{s}$, the three values of $x_{p}$ correspond to $R=4.5 \mathrm{~nm}$ (a), $R=22 \mathrm{~nm}$ (b), and $R=45 \mathrm{~nm}$ (c). The expected resonance positions of the first three sets of plasmonic modes, which in the quasistatic limit tend to the EQS modes $\left\{\mathbf{j}_{1^{\prime}}^{\|}, \mathbf{j}_{1^{\prime \prime}}^{\|}\right\} ;\left\{\mathbf{j}_{2^{\prime}}^{\|}, \mathbf{j}_{2^{\prime \prime}}^{\|}\right\}$, and $\left\{\mathbf{j}_{3^{\prime}}^{\|}, \mathbf{j}_{3^{\prime \prime}}^{\|}\right\}$, are obtained by Eq. (58), and are shown in Fig. 8 with vertical dashed lines (blue, red, and yellow, respectively).

In Fig. 8(a), it is assumed $x_{p}=0.1$. The radius is significantly smaller than the plasma wavelength and the EQS approximation alone accurately predicts the occurrence of the $W_{a b s}$ peaks through Eq. (55). The nonradiative losses cause the broadening of all peaks.

In Fig. 8(b), it is assumed $x_{p}=0.5$. The absorption peaks begin to shift with respect to the their quasistatic position, in particular the one associated to the horizontal electric dipoles $\mathbf{j}_{1^{\prime}}^{\|}$and $\mathbf{j}_{1^{\prime \prime}}^{\|}$. Nevertheless, Eq. (58), by including the radiation correction, predicts their occurrence with a relative error less than $0.5 \%$, as shown in Table V. In this table, the Q-factors obtained by Eqs. (60), (63), and (64) are also compared against their heuristic counterparts, defined in Eq. (77), and

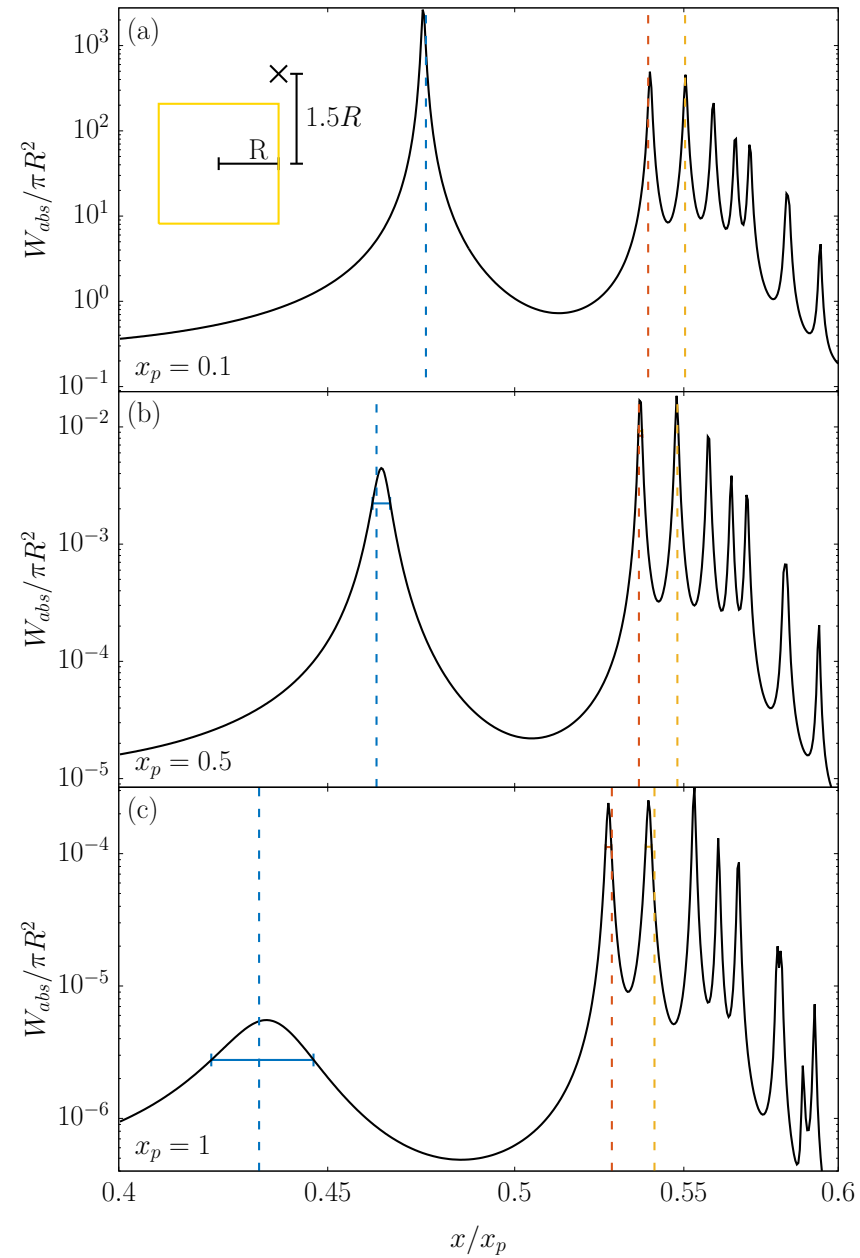

FIG. 8. Power $W_{a b s}$ absorbed by a Drude metal cylinder $(v=$ $10^{-3} \omega_{p}$ ) with radius $R=l_{c}$ and height $H=R$ as a function of $\omega / \omega_{p}=x / x_{p}$ for $x_{p}=0.1$ (a), $x_{p}=0.5$ (b), and $x_{p}=0.5$ (c). The cylinder is centered in $(0,0,0)$ and it is excited by a point source oriented along $\hat{\mathbf{y}}$, i.e., $\mathbf{E}_{i n c}(\mathbf{r})=\mathbf{N}_{o 11}^{(3)}$ at position $(R, 0,1.5 R)$, as in the inset. The resonance frequencies obtained by Eq. (58) are shown with vertical dashed lines. Horizontal lines show the FWHM of the broadest peaks.

TABLE V. Resonance frequencies $\omega_{h}$ and Q-factors $\mathrm{Q}_{h}^{\| \mathrm{r}}, \mathrm{Q}_{h}^{\| \mathrm{nr}}$, and $\mathrm{Q}_{h}^{\|}$of the first plasmonic modes of a Drude metal cylinder ( $v=10^{-3} \omega_{p}$ ) with $R=H$, and their heuristic estimates $\hat{\omega}_{h}$ and $\hat{\mathrm{Q}}_{h}$.

\begin{tabular}{|c|c|c|c|c|c|}
\hline$x_{p}$ & & & $\left\{\mathbf{j}_{1^{\prime}}^{\|}, \mathbf{j}_{1^{\prime \prime}}^{\|}\right\}$ & $\left\{\mathbf{j}_{2^{\prime}}^{\|}, \mathbf{j}_{2^{\prime \prime}}^{\|}\right\}$ & $\left\{\mathbf{j}_{3^{\prime}}^{\|}, \mathbf{j}_{3^{\prime \prime}}^{\|}\right\}$ \\
\hline \multirow[t]{6}{*}{0.5} & Theory & $\omega_{h} / \omega_{p}$ & 0.465 & 0.5363 & 0.5480 \\
\hline & Heuristic & $\hat{\omega}_{h} / \omega_{p}$ & 0.464 & 0.5364 & 0.5478 \\
\hline & Theory & $\mathrm{Q}_{h}^{\| \mathrm{r}}$ & 120 & 2279 & 2596 \\
\hline & & $\mathrm{Q}_{h}^{\| n \mathrm{nr}}$ & 465 & 536 & 547 \\
\hline & & $\mathrm{Q}_{h}^{\|}$ & 95 & 523 & 537 \\
\hline & heuristic & $\hat{\mathrm{Q}}_{h}$ & 100 & 412 & 547 \\
\hline \multirow[t]{6}{*}{1} & Theory & $\omega_{h} / \omega_{p}$ & 0.439 & 0.528 & 0.541 \\
\hline & Heuristic & $\hat{\omega}_{h} / \omega_{p}$ & 0.435 & 0.527 & 0.539 \\
\hline & Theory & $\mathrm{Q}_{h}^{\| \mathrm{r}}$ & 17.8 & 768 & 865 \\
\hline & & $\mathrm{Q}_{h}^{\| \mathrm{nr}}$ & 439 & 528 & 540 \\
\hline & & $\mathrm{Q}_{h}^{\|}$ & 17.1 & 313 & 332 \\
\hline & Heuristic & $\hat{\mathrm{Q}}_{h}$ & 17 & 310 & 292 \\
\hline
\end{tabular}


a good agreement is found. In particular, the Q-factor of the the modes $\mathbf{j}_{1^{\prime}}^{\|}$and $\mathbf{j}_{1^{\prime \prime}}^{\|}$is limited by their radiative losses, while the Q-factors of the modes $\mathbf{j}_{2^{\prime}}^{\|} \mathbf{j}_{2^{\prime \prime}}^{\|}$and $\mathbf{j}_{3^{\prime}}^{\|} \mathbf{j}_{3^{\prime \prime}}^{\|}$are limited by nonradiative damping mechanisms.

In Fig. 8(c), it is assumed $x_{p}=1$. The cylinder has radius comparable to the plasma wavelength. Despite the further shift of the peaks, the radiation corrections are still able to correctly locate the resonances, with an error less than $0.5 \%$ (see Table V). Moreover, Table V shows that while the broadening of the first peak arises from the radiative damping, both radiative and nonradiative damping contribute to the broadening of the second and third peaks. The total Q-factor is also in good agreement with the heuristic Q-factor in the investigated cases.

High-index cylinder. The power absorbed by a cylinder constituted by a nondispersive high-index dielectric with low losses is now investigated as a function of $x \sqrt{\chi}$. Three different values of $\chi$, namely $\chi=10^{4}-1 i, \chi=99-0.01 i$, and $\chi=14.45-0.1456 i$, are considered in Figs. 9(a), 9(b) and $9(\mathrm{c})$, respectively. The resonance positions obtained by Eq. (68), of the first six sets of dielectric modes, which in the quasistatic limit tend to the MQS modes $\mathbf{j}_{1}^{\perp},\left\{\mathbf{j}_{2^{\prime}}^{\perp}, \mathbf{j}_{2^{\prime \prime}}^{\perp}\right\},\left\{\mathbf{j}_{3^{\prime}}^{\perp}, \mathbf{j}_{3^{\prime \prime}}^{\perp}\right\}$, $\left\{\mathbf{j}_{4^{\prime}}^{\perp}, \mathbf{j}_{4^{\prime \prime}}^{\perp}\right\},\left\{\mathbf{j}_{5^{\prime}}^{\perp}, \mathbf{j}_{5^{\prime \prime}}^{\perp}\right\}$, and $\mathbf{j}_{6}^{\perp}$, are shown with dashed vertical lines (blue, red, yellow, violet, green, and cyan, respectively).

In Fig. 9(a), it is assumed that $\chi=10^{4}-1 i$ and $x \in$ $[0.00275,0.055]$. The size parameter is very small and even the MQS approximation alone (without corrections) accurately predicts the occurrence of the peaks by Eq. (65).

Next, in Fig. 9(b), it is assumed that $\chi=99-0.01 i$ and $x \in[0.0275,0.55]$. The peaks undergo a shift from their quasistatic positions, but Eq. (68), by taking into account the second-order radiation correction, predicts their occurrence with an error $<0.2 \%$. The resonance positions and Q-factors of $\mathbf{j}_{1}^{\perp},\left\{\mathbf{j}_{2^{\prime}}^{\perp}, \mathbf{j}_{2^{\prime \prime}}^{\perp}\right\}$, and $\mathbf{j}_{6}^{\perp}$ are compared in Table VI against the peak positions and heuristic Q-factors. Good agreement is found.

Eventually, in Fig. 9(c), a silicon cylinder with $\chi=$ $14.45-0.1456 i$ is investigated in the range $x \in[0.72,1.44]$. The size parameter is now of the order of 1 and the peaks undergo a significant shift and broadening. Nevertheless, the MQS approximation equipped with radiation corrections is able to predict through Eq. (68) the peak occurrence with an error of less than $2.5 \%$, as shown in Table VI. In this same table, the corresponding Q-factors, all dominated by radiative losses, are also shown and are very close to their heuristic counterpart. The resonance associated to $\left\{\mathbf{j}_{3^{\prime}}^{\perp}, \mathbf{j}_{3^{\prime \prime}}^{\perp}\right\}$ (vertical yellow dashed line) only corresponds to a shoulder in the $W_{a b s}$ curve due to the radiative broadening, and thus it was not possible to define its heuristic resonance position. Furthermore, the interplay of the modes $\left\{\mathbf{j}_{4^{\prime}}^{\perp}, \mathbf{j}_{4^{\prime \prime}}^{\perp}\right\}$ and $\left\{\mathbf{j}_{5^{\prime}}^{\perp}, \mathbf{j}_{5^{\prime \prime}}^{\perp}\right\}$ results in only one $W_{a b s}$ peak. For these reasons, they are not reported in Table VI.

\section{Triangular prism}

A triangular prism with basis edge $L$ and height $H=L / 2$ is now investigated. The edges and corners are rounded with a curvature radius of $L / 20$. The characteristic length is assumed to be equal to half of the edge length, i.e., $l_{c}=L / 2$. The triangular surface mesh used for the calculation of EQS resonances

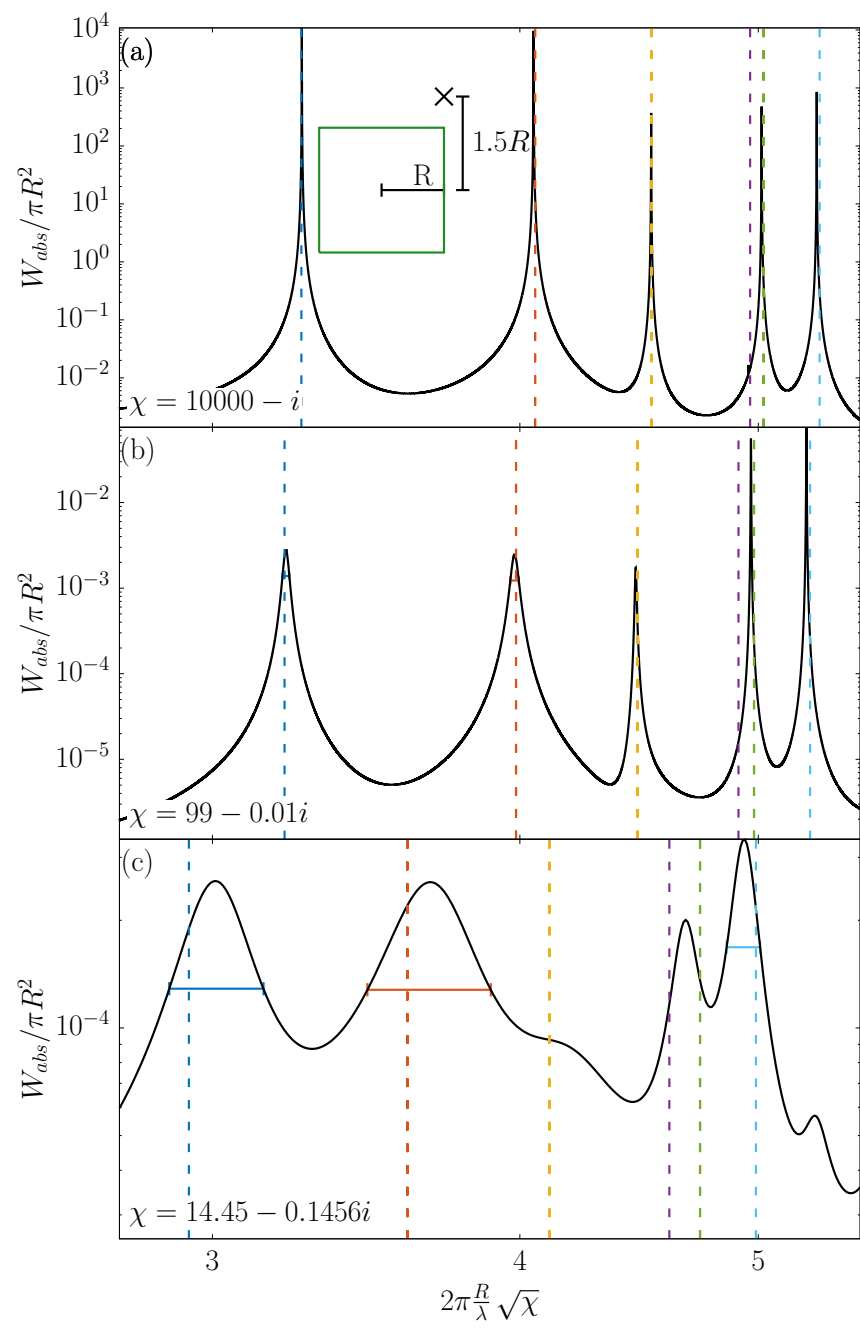

FIG. 9. Power $W_{a b s}$ absorbed by a finite-size cylinder with radius $R=l_{c}$, height $H=R$, and $\chi=$ (a) $10^{4}-1 i$, (b) $99-0.01 i$, (c) $14.45-0.1456 i$, as a function of $y=x \sqrt{\chi}$. The cylinder is centered in $(0,0,0)$ and it is excited by a point source excitation oriented along $\hat{\mathbf{y}}$, e.g., $\mathbf{E}_{i n c}(\mathbf{r})=\mathbf{N}_{\text {o11 }}^{(3)}$, at position $(R, 0,1.5 R)$. The first three resonance positions obtained by Eq. (68) are shown with vertical dashed lines. Horizontal lines show the FWHM of the broadest peaks.

TABLE VI. Resonance position $x_{h} \sqrt{\chi}$ and Q-factors $\mathrm{Q}_{h}^{\perp \mathrm{r}}, \mathrm{Q}_{h}^{\perp \mathrm{nr}}$, and $\mathrm{Q}_{h}^{\perp}$ of the dielectric modes $1,2,6$ of a dielectric cylinder $(H=$ $R$ ) with different susceptibility $\chi$, and their corresponding heuristic estimates $\hat{x}_{h} \sqrt{\chi}$ and $\hat{\mathrm{Q}}_{h}$.

\begin{tabular}{lccccc}
\hline \hline$\chi$ & & & $\left\{\mathbf{j}_{1}^{\perp}, \mathbf{j}_{1^{\prime}}^{\perp}\right\}$ & $\left\{\mathbf{j}_{2}^{\perp}, \mathbf{j}_{2^{\prime}}^{\perp}\right\}$ & $\mathbf{j}_{6}^{\perp}$ \\
\hline 99-0.01i & Theory & $x_{h} \sqrt{\chi}$ & 3.209 & 3.986 & 5.248 \\
& Heuristic & $\hat{x}_{h} \sqrt{\chi}$ & 3.214 & 3.980 & 5.230 \\
& Theory & $\mathrm{Q}_{h}^{\perp \mathrm{r}}$ & 147 & 118 & 3117 \\
& & $\mathrm{Q}_{h}^{\perp \mathrm{nr}}$ & $10^{4}$ & $10^{4}$ & $10^{4}$ \\
& & $\mathrm{Q}_{h}^{\perp}$ & 144 & 116 & 2615 \\
& & & 156 & 126 & 2615 \\
& Heuristic & $\hat{\mathrm{Q}}_{h}$ & 156 & \\
\hline \multirow{2}{*}{$14.45-0.1456 i$} & Theory & $x_{h} \sqrt{\chi}$ & 2.935 & 3.600 & 4.989 \\
& Heuristic & $\hat{x}_{h} \sqrt{\chi}$ & 3.007 & 3.677 & 4.938 \\
& Theory & $\mathrm{Q}_{h}^{\perp} \approx \mathrm{Q}_{h}^{\perp \mathrm{r}}$ & 10.74 & 8.87 & 32.6 \\
& Heuristic & $\hat{\mathrm{Q}}_{h}$ & 11.5 & 8.67 & 31.9 \\
\hline \hline
\end{tabular}


(a)

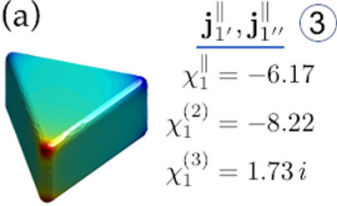

(b)

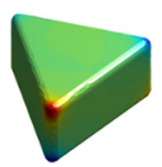

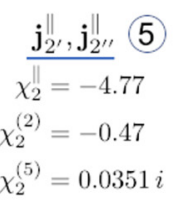

(c)

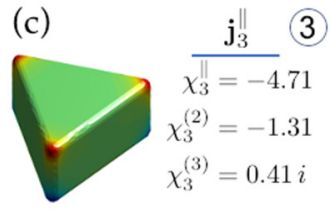

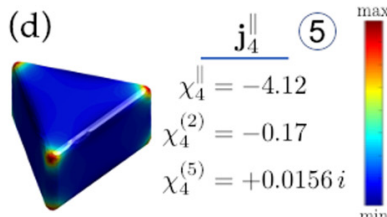

FIG. 10. Catalog of plasmonic resonances of a triangular prism with basis edge $L$ and height $H=L / 2$, assuming $l_{c}=L / 2$. The electroquasistatic current density modes $\mathbf{j}_{h}^{\|}$are ordered according to their eigenvalue $\chi_{h}^{\|}$. Their normal component on $\partial \Omega$ is shown with a surface color plot. The second-order correction $\chi_{h}^{(2)}$, and the nonvanishing imaginary correction $\chi_{h}^{(n)}$ of the lowest order $n_{i}$ are shown on the right of each panel. The value of $n_{i}$ is also highlighted on the top right of each panel.

has 2349 nodes and 4694 triangles, while the hexahedral mesh used for the calculation of MQS resonances has 2520 nodes, 2025 hexahedra, and 3592 edges.

\section{Catalog of plasmonic resonances}

The catalog of the first four plasmonic resonances of a triangular prism is presented in Fig. 10. The first two degenerate EQS current modes are $\left\{\mathbf{j}_{1^{\prime}}^{\|}, \mathbf{j}_{1^{\prime \prime}}^{\|}\right\}$, depicted in Fig. 10(a). They represent two electric dipoles oriented along mutually orthogonal directions and are indeed bright. The third-order correction is proportional to the squared magnitude of their electric dipole moment, according to Eq. (33).

The next EQS modes $\left\{\mathbf{j}_{2^{\prime}}^{\|}, \mathbf{j}_{2^{\prime \prime}}^{\|}\right\}$exhibit a quadrupolar character. They are described in Fig. 10(b). They are dark; for this reason, the third-order correction $\chi_{2}^{(3)}$ vanishes. Nevertheless, they have a nonvanishing electric quadrupole tensor, and thus the fifth-order correction $\chi_{2}^{(5)}$ is nonzero according to Eq. (34).

The successive EQS mode $\mathbf{j}_{3}^{\|}$is bright and corresponds to a vertical electric dipole. The third-order correction $\chi_{3}^{(3)}$ is proportional to the squared magnitude of its electric dipole moment of $\mathbf{j}_{3}^{\|}$.

The next EQS mode $\mathbf{j}_{4}^{\|}$exhibits a quadrupolar character. It is shown in Fig. 10(d). It is dark, and thus the third-order correction $\chi_{4}^{(3)}$ vanishes. Nevertheless, it has a nonvanishing electric quadrupole tensor and the fifth-order correction $\chi_{4}^{(5)}$ is nonzero and given by Eq. (34).

\section{Catalog of dielectric resonances}

The catalog of the first six dielectric resonances of the finite-size triangular prism is presented in Fig. 11. The mode $\mathbf{j}_{1}^{\perp}$, shown in Fig. 11(a), is associated to the lowest MQS eigenvalue. It is a magnetic dipole oriented along the vertical axis. The current mode $\mathbf{j}_{1}^{\perp}$ is an $\mathbb{A}^{\perp}$ mode, since the longitudinal part of the vector potential generated by $\mathbf{j}_{1}^{\perp}$ is numerically negligible. This fact exemplifies that $\mathbb{A}^{\perp}$ modes can be also found in nonrotationally symmetric objects. Thus, the second-order correction $\kappa_{1}^{(2)}$ is simply given by Eq. (43). The third-order correction is proportional to the squared magnitude of the magnetic dipole moment of $\mathbf{j}_{1}^{\perp}$, accordingly to Eq. (48).

The next two degenerate modes $\left\{\mathbf{j}_{2^{\prime}}^{\perp}, \mathbf{j}_{2^{\prime \prime}}^{\perp}\right\}$ are in-plane magnetic dipoles; one of them is shown in Fig. 11(b). They are $\mathbb{A}^{\perp}$ modes with a nonvanishing magnetic dipole moment and the considerations made for $\mathbf{j}_{1}^{\perp}$ also apply here.

The next mode $\mathbf{j}_{3}^{\perp}$ is shown in Fig. 11(c). The vector potential generated by the current mode $\mathbf{j}_{3}^{\perp}$ has a nonvanishing longitudinal component. In particular, the magnetostatic interaction energy between $\mathbf{j}_{3}^{\perp}$ and the vertical EQS current modes $\mathbf{j}_{3}^{\|}$, shown in Fig. 10(c), contributes to the second-order correction $\kappa_{3}^{(2)}$. Since the mode $\mathbf{j}_{3}^{\perp}$ has zero magnetic dipole, the third-order correction vanishes. The imaginary correction of the lowest order is $\kappa_{3}^{(5)}$, and it is determined by the effective dipole resulting from the interference between the toroidal dipole moment $\mathbf{P}_{\mathrm{E} 2 \mid 3}^{\perp}$ and the electric dipole moment of the second-order correction $\mathbf{P}_{\mathrm{E} \mid 3}^{(2)}$.
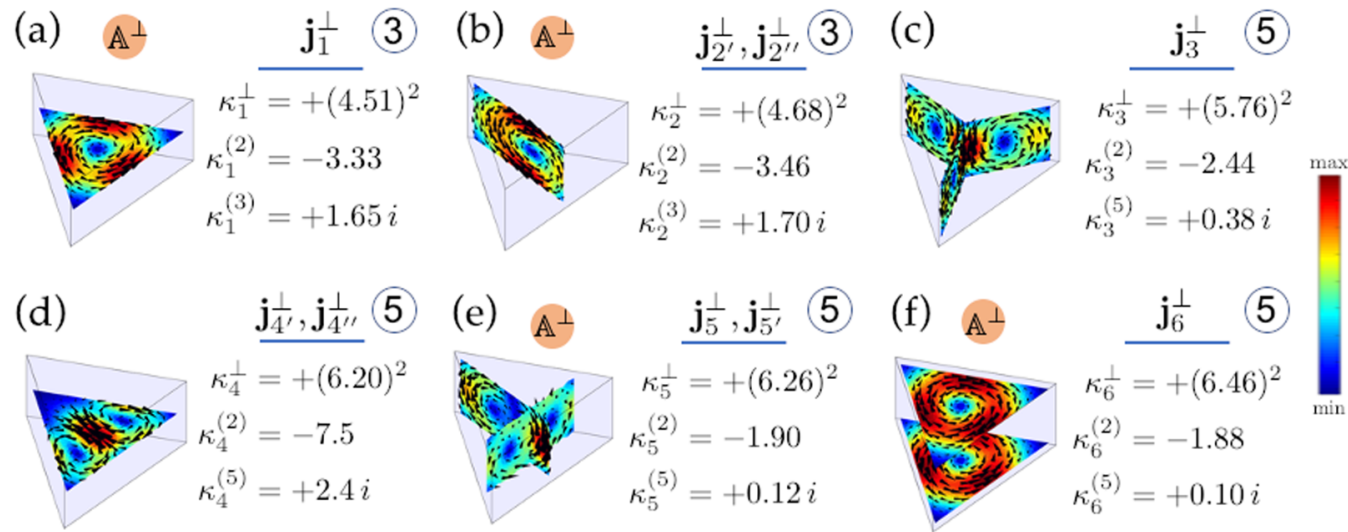

FIG. 11. Catalog of dielectric resonances of a triangular prism with basis edge $L$ and height $H=L / 2$, assuming $l_{c}=L / 2$. The magnetoquasistatic current density modes are ordered according to their eigenvalue $\kappa_{h}^{\perp}$. The second-order correction $\kappa_{h}^{(2)}$ and the nonvanishing imaginary correction $\kappa_{h}^{(n)}$ of lowest order $n_{i}$ are shown on the right of the corresponding panel, while the value of $n_{i}$ is also highlighted on the top right enclosed in a circle. The current modes generating a transverse vector potential are labeled with $\mathbb{A}^{\perp}$. 
The next degenerate modes are $\left\{\mathbf{j}_{4^{\prime}}^{\perp}, \mathbf{j}_{4^{\prime \prime}}^{\perp}\right\}$, described in Fig. 11(d). They generate a vector potential with a nonvanishing longitudinal component. Specifically, the magnetostatic interaction energy between them and two EQS electric dipole modes $\mathbf{j}_{1^{\prime}}^{\|}, \mathbf{j}_{1^{\prime \prime}}^{\|}$is nonvanishing and contributes to the secondorder correction $\kappa_{4}^{(2)}$ in Eq. (41). Since $\mathbf{j}_{4^{\prime}}^{\perp}, \mathbf{j}_{4^{\prime \prime}}^{\perp}$ have zero magnetic dipole moment, $\kappa_{4}^{(3)}=0$, while the fifth-order correction is nonvanishing and determined by the multipoles $\mathbf{P}_{\mathrm{E} 2 \mid 4}^{\perp}$ and $\mathbf{P}_{\mathrm{E} \mid 4}^{(2)}$.

The subsequent two sets of MQS modes, i.e., $\left\{\mathbf{j}_{5^{\prime}}^{\perp}, \mathbf{j}_{5^{\prime \prime}}^{\perp}\right\}$ and $\mathbf{j}_{6}^{\perp}$, shown in Figs. 11(e) and 11(f), are $\mathbb{A}^{\perp}$ modes. Therefore, the second-order correction is given by Eq. (43). These modes exhibit zero magnetic dipole moment and thus have vanishing third-order correction. The fifth-order corrections can be calculated by Eq. (52), and in both cases originate from the magnetic quadrupole of the MQS mode.

\section{CONCLUSIONS}

Maxwell's equations provide an exhaustive description of classical electromagnetic phenomena, from the simplest to the most sophisticated ones. However, in many applications, wave phenomena occurring at short timescales are of no practical concern, and the fields may be described by either the electroquasistatic or the magnetoquasistatic approximation, i.e., the approximations behind the description of capacitors and inductors. This is also the case for light scattering: Resonances in metal or high-index objects, assumed much smaller than the vacuum wavelength, may be respectively described by the electroquasistatic or the magnetoquasistatic approximation. Unfortunately, both approximations are unable to predict the frequency shift and radiative Q-factors, which arise from the coupling with the radiation.

In this paper, closed-form expressions for the radiation corrections to the real and imaginary parts of both electroquasistatic and magnetoquasistatic eigenvalues and of the corresponding modes are derived. These corrections only depend on the quasistatic current mode distribution. The expression of the radiation corrections are greatly simplified if the magnetoquasistatic mode generates a transverse vector potential, namely with vanishing normal component to the surface of the object $\left(\mathbb{A}^{\perp}\right.$ mode).

Closed form expressions of the frequency shift and the radiative Q-factor of both plasmonic and dielectric modes of small objects are introduced, where the dependencies on the material and the size of the object are factorized. The relative frequency shift of any mode is a quadratic function of the size parameter at the quasistatic resonance, whose prefactor depends on the ratio between the second-order correction and the quasistatic eigenvalue. The radiative $\mathrm{Q}$-factor is an inverse power function of the size parameter whose exponent is the order $n_{i} \geqslant 3$ of the first nonvanishing imaginary correction, while the prefactor is the ratio between the the static eigenvalue and its $n_{i}$ th-order imaginary correction. Specifically, the prefactor only depends on the quasistatic eigenvalue and on the multipolar components of the quasistatic modes and their corrections.
The scattering modes of small objects can be then naturally classified in two catalogs of plasmonic and dielectric resonances, containing the essential information to analyze and engineer the electromagnetic scattering from small objects. In these tables, the resonances are sorted accordingly to their real quasistatic eigenvalue and characterized by the second-order correction and by the nonvanishing imaginary correction of the lowest order, i.e., $n_{i}$. All the quantities contained in these tables depend neither on the size nor on the permittivity but only on the quasistatic mode morphology.

The introduced expressions for the resonance frequency and Q-factor are successfully validated by predicting the resonance peaks and their broadening in the absorption spectra of a sphere and a finite-size cylinder. Both Drude metals and high-index dielectric are considered.

\section{APPENDIX A: MULTIPOLES}

The electric dipole moment $\mathbf{P}_{\mathrm{E} \mid h}^{\|}$of the $h$ th electroquasistatic current mode $\mathbf{j}_{h}^{\|}$is defined as

$$
\mathbf{P}_{\mathrm{E} \mid h}^{\|}=\int_{\tilde{\Omega}} \mathbf{j}_{h}^{\|} d \tilde{V}=\oint_{\partial \tilde{\Omega}}\left(\mathbf{j}_{h}^{\|} \cdot \hat{\mathbf{n}}\right) \tilde{\mathbf{r}} d \tilde{S}
$$

and the electric quadrupole tensor $\overleftrightarrow{\mathbf{Q}}_{\mathrm{E} \mid h}^{\|}$as

$$
\overleftrightarrow{\mathbf{Q}}_{\mathrm{E} \mid h}^{\|}=\int_{\tilde{\Omega}} \tilde{\mathbf{r}} \mathbf{j}_{h}^{\|}+\mathbf{j}_{h}^{\|} \tilde{\mathbf{r}} d \tilde{V}=\oint_{\partial \tilde{\Omega}}\left(\mathbf{j}_{h}^{\|} \cdot \hat{\mathbf{n}}\right) \tilde{\mathbf{r}} \tilde{\mathbf{r}} d \tilde{S}
$$

where, with respect to standard definitions of electric multipoles [75], the prefactor $1 /(j \omega)$ is omitted.

The magnetic dipole moment $\mathbf{P}_{\mathrm{M} \mid h}^{\perp}$ of the $h$ th magnetoquasistatic current mode $\mathbf{j}_{h}^{\perp}$ is defined as

$$
\mathbf{P}_{\mathrm{M} \mid h}^{\perp}=\frac{1}{2} \int_{\tilde{\Omega}} \tilde{\mathbf{r}} \times \mathbf{j}_{h}^{\perp} d \tilde{V},
$$

the toroidal dipole $\mathbf{P}_{\mathrm{E} 2 \mid h}^{\perp}$ as [75]

$$
\mathbf{P}_{\mathrm{E} 2 \mid h}^{\perp}=\frac{1}{6} \int_{\tilde{\Omega}} \tilde{\mathbf{r}} \times \mathbf{j}_{h}^{\perp} \times \tilde{\mathbf{r}} d \tilde{V},
$$

and the magnetic quadrupole $\overleftrightarrow{\mathbf{Q}}_{\mathbf{M} \mid h}^{\perp}$ tensor as

$$
\overleftrightarrow{\mathbf{Q}}_{\mathrm{M} \mid h}^{\perp}=\frac{1}{3} \int_{\tilde{\Omega}}\left[\left(\tilde{\mathbf{r}} \times \mathbf{j}_{h}^{\perp}\right) \tilde{\mathbf{r}}+\tilde{\mathbf{r}}\left(\tilde{\mathbf{r}} \times \mathbf{j}_{h}^{\perp}\right)\right] d \tilde{V}
$$

\section{APPENDIX B: QUASISTATIC MODES OF A SPHERE}

A sphere of radius $R$ is considered, and the characteristic dimension is assumed equal to the radius $l_{c}=R$. The formulas of radiation corrections presented in this section can be extrapolated by perturbing the denominators of the Mie coefficients in the neighborhood of their EQS and MQS resonances, and they can also be directly obtained from the Padè expansion of the Mie coefficients found by Tzarouchis and Sihvola in Refs. [76,77]. 


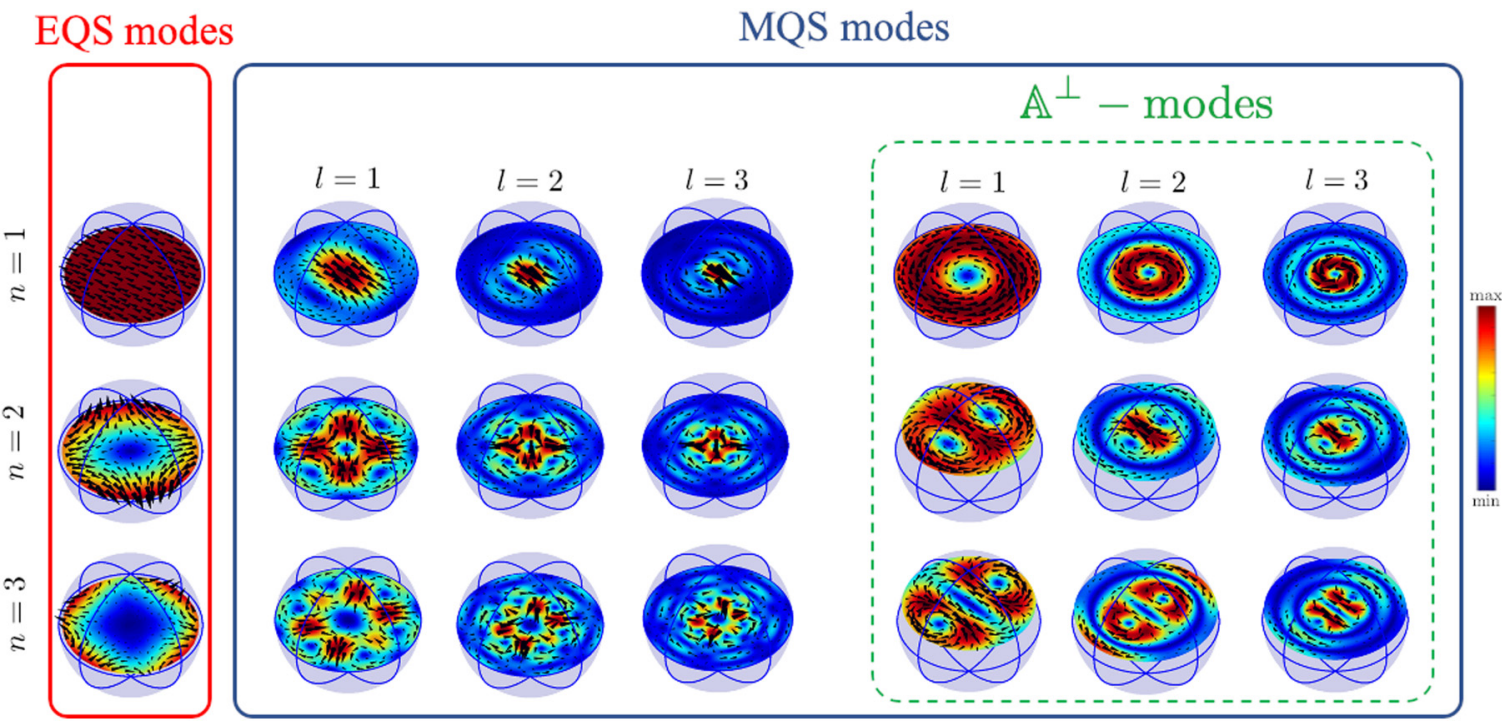

FIG. 12. Quasistatic modes of a sphere. Electroquasistatic modes $\mathbf{j}_{e 1 n}^{\|}$with $n=1,2,3$. The magnetoquasistatic modes are divided into two subsets: the TM MQS modes $\mathbf{j}_{e 1 n l}^{\perp \mathrm{TM}}(\tilde{r}, \theta, \phi)$ with $n=1,2,3$ and $l=1,2,3$, and the TE modes $\left(\mathbb{A}^{\perp} \operatorname{modes}\right) \mathbf{j}_{o l n l}^{\perp \mathrm{TE}}(\tilde{r}, \theta, \phi)$ with $n=1,2,3$ and $l=1,2,3$.

\section{Electroquasistatic modes}

In particular, the EQS eigenvalues of a sphere and their radiative corrections are [76,77]

$$
\begin{aligned}
\chi_{n}^{\|} & =-\frac{2 n+1}{n}, \\
\chi_{n}^{(2)} & =-\frac{2}{n^{2}} \frac{(n+1)(2 n+1)}{(3+2 n)(2 n-1)}, \\
\chi_{n}^{(2 n+1)} & =+i \frac{(n+1)}{[n(2 n-1) ! !]^{2}},
\end{aligned}
$$

where $n=1,2,3, \ldots$ and $(2 n-1) ! !=1 \times 3 \times 5 \times \cdots \times$ $(2 n-1)$. Each eigenvalue $\chi_{n}^{\|}$is associated to a set of $2 n+1$ degenerate current modes with $m=0,1,2, \ldots, n$ and different parities, whose analytic expressions are

$$
\begin{aligned}
\mathbf{j}_{e_{m}}^{\|}= & \frac{1}{\sqrt{\alpha_{m n}}}\left[\begin{array}{c}
\cos m \phi \\
\sin m \phi
\end{array}\right) n P_{n}^{m}(\cos \theta) \hat{\boldsymbol{r}}+\frac{d P_{n}^{m}(\cos \theta)}{d \theta} \\
& \left.\times \cos m \varphi \hat{\boldsymbol{\theta}}+\left(\begin{array}{l}
-\sin m \phi \\
+\cos m \phi
\end{array}\right) m \frac{P_{n}^{m}(\cos \theta)}{\sin \theta} \hat{\boldsymbol{\phi}}\right], \\
\alpha_{m n}= & 2 \pi\left(\delta_{m}+1\right) \frac{(m+n) !(n+1)^{n-1}}{(2 n+1)(n-m) ! n^{n-2}},
\end{aligned}
$$

where the subscripts $e$ and $o$ denote even and odd, and $P_{n}^{m}$ are the associated Legendre functions of the first kind of degree $n$ and order $m$ as defined and normalized in Ref. [68]. The prefactor $\alpha_{m n}$ guarantees that $\left\|\mathbf{j}_{e^{m}}^{\|}\right\|=1$. As an example, the EQS modes $\mathbf{j}_{e 1 n}^{\|}$with $n=1,2,3$ are shown in Fig. 12 .

The radiative quality factor of plasmonic resonances is obtained by using Eqs. (B1a) and (B1c) in Eq. (60):

$$
\mathrm{Q}_{n}^{\| \mathrm{r}}=n \frac{[(2 n+1) ! !]^{2}}{(n+1)(2 n+1)}\left(\frac{1}{x_{h}}\right)^{2 n+1} .
$$

For instance, for electric dipole, quadrupole, octupole is

$$
\mathrm{Q}_{1}^{\| \mathrm{r}}=\frac{3}{2} \frac{1}{x_{1}^{3}}, \quad \mathrm{Q}_{2}^{\| \mathrm{r}}=\frac{30}{x_{2}^{5}}, \quad \mathrm{Q}_{3}^{\| \mathrm{r}}=\frac{1181}{x_{3}^{7}} .
$$

Equation (B3) coincides with the formulas provided by Colas des Francs in Ref. [73].

\section{Magnetoquasistatic modes}

The MQS modes are divided in two sets. The first set is composed of the current modes which have no radial component. Since the corresponding electric field has the same property, these modes are called transverse electric (TE) MQS modes. These current modes also generate a vector potential with vanishing normal component to the object's boundary, so they are $\mathbb{A}^{\perp}$ modes. The second set of current modes generate a magnetic field with vanishing radial component so they are called transverse magnetic (TM) MQS modes. These current modes generate instead a vector potential with nonvanishing normal component to the object's boundary.

\section{a. TE MQS modes}

The first set of MQS modes is made by the set of TE MQS modes which coincides with the $\mathbb{A}^{\perp}$ mode of a sphere. Their eigenvalues and the corresponding corrections are [76,77]

$$
\begin{gathered}
\kappa_{n l}^{\perp \mathrm{TE}}=\left(z_{n-1, l}\right)^{2}, \\
\kappa_{n l}^{\mathrm{TE}(2)}=-\frac{2 n+1}{2 n-1}, \\
\kappa_{n l}^{\mathrm{TE}(2 n+1)}=+i \frac{2}{[(2 n-1) ! !]^{2}},
\end{gathered}
$$

where $z_{n l}$ denotes the $l$ th zero of the spherical Bessel function $j_{n}$. Each eigenvalue $\kappa_{n l}^{\perp \mathrm{TE}}$ is associated to a set of $2 n+1$ degenerate current modes with $m=0,1,2, \ldots, n$ and with 
even and odd parity, whose analytic expression is

$$
\begin{aligned}
\underset{\mathbf{j}_{e}}{\perp \mathrm{TE}}(\tilde{r}, \theta, \phi)= & \frac{1}{\sqrt{\beta_{m n l}}}\left[m\left(\begin{array}{c}
-\sin m \phi \\
+\cos m \phi
\end{array}\right) \frac{P_{n}^{m}(\cos \theta)}{\sin \theta} \hat{\boldsymbol{\theta}}\right. \\
& \left.-\left(\begin{array}{c}
\cos m \phi \\
\sin m \phi
\end{array}\right) \frac{d P_{n}^{m}(\cos \theta)}{d \theta} \hat{\boldsymbol{\varphi}}\right] j_{n}\left(z_{n-1, l} \tilde{r}\right) \quad(\mathrm{B} 6) \\
\beta_{m n l}= & 2 \pi\left(\delta_{m}+1\right) \frac{n(n+1)(m+n) !}{2(2 n+1)(n-m) !} j_{n}^{2}\left(z_{n-1, l}\right),
\end{aligned}
$$

where the prefactor $\beta_{m n l}$ guarantees that $\underset{\substack{e_{m} \\ e_{m l}}}{\perp \mathrm{TE}} \|=1$. As an example, the odd MQS modes $\mathbf{j}_{o 1 n l}^{\perp \mathrm{TE}}(\tilde{r}, \theta, \phi)$ with $m=1, n=$ $1,2,3$, and $l=1,2,3$ are shown in Fig. 12.

The radiative quality factor of the TE MQS modes is obtained by combining Eqs. (B5a) and (B5c) with (70):

$$
\mathrm{Q}_{n, l}^{\perp \mathrm{TEr}}=\frac{\left[z_{n-1, l}(2 n-1) ! !\right]^{2}}{2}\left(\frac{1}{x_{n l}}\right)^{2 n+1} .
$$

For instance, for the magnetic dipole and quadrupole,

$$
\mathrm{Q}_{1,1}^{\perp \mathrm{TEr}}=\frac{\pi^{2}}{2} \frac{1}{x_{1}^{3}} \approx \frac{4.9}{x_{1,1}^{3}} ; \mathrm{Q}_{2,1}^{\perp \mathrm{TEr}}=\frac{9 z_{1,1}^{2}}{2} \frac{1}{x_{2,1}^{5}} \approx \frac{90.7}{x_{2,1}^{5}} .
$$

\section{b. TM MQS modes}

The second set of MQS modes is made by the set of TM MQS modes. Their eigenvalues and the corresponding corrections are [76,77]

$$
\begin{aligned}
\kappa_{n l}^{\perp \mathrm{TM}} & =\left(z_{n l}\right)^{2}, \\
\kappa_{n l}^{\mathrm{TM}(2)} & =-\frac{n+2}{n}, \\
\kappa_{n l}^{\mathrm{TM}(2 n+3)} & =+i \frac{2}{n^{2}[(2 n-1) ! !]^{2}} .
\end{aligned}
$$

Each eigenvalue $\kappa_{n l}^{\perp \mathrm{TM}}$ is associated to a set of $2 n+1$ degenerate current modes with $m=0,1,2, \ldots, n$ and with even and odd parity whose analytic expression is

$$
\begin{aligned}
& \mathbf{j}_{e_{m}}^{\perp \mathrm{TM}}(\tilde{r}, \theta, \phi) \\
&= \frac{1}{\sqrt{\gamma_{m n l}}}\left\{\left(\begin{array}{c}
\cos m \phi \\
\sin m \phi
\end{array}\right) n(n+1) P_{n}^{m}(\cos \theta) \frac{j_{n}\left(z_{n l} \tilde{r}\right)}{z_{n l} \tilde{r}} \hat{\boldsymbol{r}}\right. \\
&+\left(\begin{array}{c}
\cos m \phi \\
\sin m \phi
\end{array}\right) \frac{d P_{n}^{m}(\cos \theta)}{d \theta} \frac{1}{z_{n l} \tilde{r}} \frac{d}{d \tilde{r}}\left[\tilde{r} j_{n}\left(z_{n l} \tilde{r}\right)\right] \hat{\boldsymbol{\theta}} \\
&\left.+m\left(\begin{array}{l}
-\sin m \phi \\
+\cos m \phi
\end{array}\right) \frac{P_{n}^{m}(\cos \theta)}{\sin \theta} \frac{1}{z_{n l} \tilde{r}} \frac{d}{d \tilde{r}}\left[\tilde{r} j_{n}\left(z_{n l} \tilde{r}\right)\right] \hat{\boldsymbol{\varphi}}\right\}, \\
& \gamma_{m n l}= \\
& \quad \pi\left(1+\delta_{m}\right) \frac{n(n+1)(n+m) !}{(2 n+1)^{2}(n-m) !} \\
& \times\left[(1+n) j_{n-1}^{2}\left(z_{n l}\right)+n j_{n+1}^{2}\left(z_{n l}\right)\right], \quad \text { (B11) }
\end{aligned}
$$

where the prefactor $\gamma_{m n l}$ guarantees that $\left\|\mathbf{j}_{e_{m n l}}^{\perp \mathrm{TM}}\right\|=1$. The subscripts $e$ and $o$ denote even and odd, and $P_{n}^{m}(\cdot)$ are the associated Legendre function of the first kind of degree $n$ and order $m$.

The MQS modes $\mathbf{j}_{e 1 n l}^{\perp \mathrm{TM}}(\tilde{r}, \theta, \phi)$ with $n=1,2,3$ and $l=$ 1, 2, 3 are shown in Fig. 12.

The radiative quality factor of the TE MQS modes is obtained by combining Eqs. (B9a) and (B9c) with Eq. (70):

$$
Q_{n l}^{\perp \mathrm{TMr}}=\frac{\left[n z_{n, l}(2 n-1) ! !\right]^{2}}{2}\left(\frac{1}{x_{n l}}\right)^{2 n+3} .
$$

As an example the toroidal electric dipole mode has radiative Q factor:

$$
Q_{1,1}^{\perp \mathrm{TMr}}=\frac{z_{1,1}^{2}}{2} \frac{1}{x_{1,1}^{5}} \approx \frac{10.1}{x_{1,1}^{5}} .
$$

\section{APPENDIX C: POINT SOURCE EXCITATION}

Following Ref. [68], the explicit expression of the vector spherical wave function $\mathbf{N}_{e_{11}}^{(3)}$ of the radiative kind is

$$
\begin{aligned}
\mathbf{N}_{e_{11}}^{(3)}= & -\left(\begin{array}{c}
\cos \phi \\
\sin \phi
\end{array}\right)\left(-\frac{i}{\left(k_{0} r\right)^{3}}+\frac{1}{\left(k_{0} r\right)^{2}}\right) 2 \sin \theta e^{-i k_{0} r} \hat{\boldsymbol{r}}+\left(\begin{array}{c}
\cos \phi \\
\sin \phi
\end{array}\right) \cos \theta\left(-\frac{i}{\left(k_{0} r\right)^{3}}+\frac{1}{\left(k_{0} r\right)^{2}}+\frac{i}{\left(k_{0} r\right)}\right) e^{-i k_{0} r} \hat{\boldsymbol{\theta}} \\
& +\left(\begin{array}{c}
-\sin \phi \\
+\cos \phi
\end{array}\right)\left(-\frac{i}{\left(k_{0} r\right)^{3}}+\frac{1}{\left(k_{0} r\right)^{2}}+\frac{i}{\left(k_{0} r\right)}\right) e^{-i k_{0} r} \hat{\boldsymbol{\phi}},
\end{aligned}
$$

where the subscripts $e$ and $o$ denote even and odd. Equation (C1) is proportional to the electric field radiated in vacuum by the electric dipole $\mathbf{p}=\hat{\mathbf{x}}$ when the parity index is $e$ and by $\mathbf{p}=\hat{\mathbf{y}}$ when the parity index is odd. Those fields can be exactly obtained by multiplying Eq. $(\mathrm{C} 1)$ by the factor $i \frac{1}{4 \pi \varepsilon_{0}} \frac{\omega^{3}}{c^{3}}$.

\section{APPENDIX D: DERIVATION OF RADIATION CORRECTIONS FOR PLASMONIC MODES AND RESONANCES}

In this appendix the derivation of the radiation corrections for the plasmonic modes and resonances is carried out in detail, by matching same order terms in Eq. (26). 


\section{Zero order}

Collecting the zeroth-order terms in Eq. (26), the following integrodifferential equation is obtained:

$$
\mathbf{u}_{h}^{(0)}(\tilde{\mathbf{r}})+\chi_{h}^{(0)} \frac{1}{4 \pi} \tilde{\nabla} \oint_{\partial \tilde{\Omega}} \Delta \tilde{\mathbf{r}}^{-1} u_{n, h}^{(0)}\left(\tilde{\mathbf{r}}^{\prime}\right) d \tilde{S}^{\prime}=\mathbf{0} \quad \forall \tilde{\mathbf{r}} \in \tilde{\Omega}
$$

The problem (D1) is the EQS auxiliary eigenvalue problem introduced in Eq. (1) of the main paper. Thus,

$$
\chi_{h}^{(0)}=\chi_{h}^{\|}
$$

and the zeroth-order current density field is purely longitudinal:

$$
\mathbf{u}_{h}^{(0)}(\tilde{\mathbf{r}})=\mathbf{j}_{h}^{\|}(\tilde{\mathbf{r}}) \quad \forall \tilde{\mathbf{r}} \in \Omega .
$$

\section{First order}

Matching the first-order terms in Eq. (26), the following integrodifferential equation is obtained:

$$
-4 \pi \mathbf{u}_{h}^{(1)}(\tilde{\mathbf{r}})+i \chi_{h}^{\|} \tilde{\nabla} \oint_{\partial \tilde{\Omega}} j_{n, h}^{\|}(\tilde{\mathbf{r}}) d \tilde{S}^{\prime}-\chi_{h}^{(1)} \tilde{\nabla} \oint_{\partial \tilde{\Omega}} \Delta \tilde{\mathbf{r}}^{-1} j_{n, h}^{\|}\left(\tilde{\mathbf{r}}^{\prime}\right) d \tilde{S}^{\prime}-\chi_{h}^{\|} \tilde{\nabla} \oint_{\partial \tilde{\Omega}} \Delta \tilde{\mathbf{r}}^{-1} u_{n, h}^{(1)}\left(\tilde{\mathbf{r}}^{\prime}\right) d \tilde{S}^{\prime}=0 \quad \forall \tilde{\mathbf{r}} \in \tilde{\Omega},
$$

where the second term from the left vanishes due to the charge neutrality condition [Eq. (3) of the main paper], and Eqs. (D2) and (D3) were used. Thus, Eq. (D4) can be rewritten as

$$
\mathbf{u}_{h}^{(1)}+\chi_{h}^{\|}\left(\frac{1}{4 \pi} \tilde{\nabla} \oint_{\partial \tilde{\Omega}} \Delta \tilde{\mathbf{r}}^{-1} u_{n, h}^{(1)}\left(\tilde{\mathbf{r}}^{\prime}\right) d \tilde{S}^{\prime}\right)=-\chi_{h}^{(1)} \frac{1}{4 \pi} \tilde{\nabla} \oint_{\partial \tilde{\Omega}} \Delta \tilde{\mathbf{r}}^{-1} j_{n, h}^{\|}\left(\tilde{\mathbf{r}}^{\prime}\right) d \tilde{S}^{\prime}, \quad \forall \tilde{\mathbf{r}} \in \tilde{\Omega} .
$$

Since $\chi_{h}^{\|}$is an eigenvalue of the left-hand side of Eq. (D5), a solution of Eq. (D5) exists only under the condition that its right-hand side is orthogonal to the corresponding current mode $\mathbf{j}_{h}^{\|}$, accordingly to the scalar product introduced in Eq. (5) of the main paper. This is the so-called normal solvability condition of Fredholm integral equations [61,62]. Consequently,

$$
\chi_{h}^{(1)} \frac{1}{4 \pi} \int_{\tilde{\Omega}} \mathbf{j}_{h}^{\|}(\tilde{\mathbf{r}}) \cdot\left(\tilde{\nabla} \oint_{\partial \tilde{\Omega}} \Delta \tilde{\mathbf{r}}^{-1} j_{n, h}^{\|}\left(\tilde{\mathbf{r}}^{\prime}\right) d \tilde{S}^{\prime}\right) d \tilde{V}=\frac{\chi_{h}^{(1)}}{\chi_{h}^{\|}}\left\|\mathbf{j}_{h}^{\|}\right\|^{2}=0,
$$

where Eq. (D1) has been used. Thus, the integral equation (D5) is only solvable if

$$
\chi_{h}^{(1)}=0 .
$$

Regardless of the object's shape, the first-order correction to EQS eigenvalues vanishes. As a result of Eq. (D7), the integral equation (D5) only admits the trivial solution

$$
\mathbf{u}_{h}^{(1)}(\tilde{\mathbf{r}})=\mathbf{0} \quad \forall \tilde{\mathbf{r}} \in \Omega
$$

\section{Second order}

Collecting the second-order terms in Eq. (26), the following integrodifferential equation is obtained (div- and curl-free functions within $\Omega$ which have nonzero normal component on $\partial \tilde{\Omega}$ ):

$$
\begin{aligned}
\mathbf{u}_{h}^{(2)} & +\chi_{h}^{\|} \frac{1}{4 \pi} \tilde{\nabla} \oint_{\partial \tilde{\Omega}} \Delta \tilde{\mathbf{r}}^{-1} u_{n, h}^{(2)}\left(\tilde{\mathbf{r}}^{\prime}\right) d \tilde{S}^{\prime} \\
& =-\chi_{h}^{(2)} \frac{1}{4 \pi} \tilde{\nabla} \oint_{\partial \tilde{\Omega}} \Delta \tilde{\mathbf{r}}^{-1} j_{n, h}^{\|}\left(\tilde{\mathbf{r}}^{\prime}\right) d \tilde{S}^{\prime}+\chi_{h}^{\|} \frac{1}{4 \pi} \tilde{\nabla} \oint_{\partial \tilde{\Omega}} \frac{\Delta \tilde{\mathbf{r}}}{2 !} j_{n, h}^{\|}\left(\tilde{\mathbf{r}}^{\prime}\right) d \tilde{S}^{\prime}+\chi_{h}^{\|} \frac{1}{4 \pi} \int_{\tilde{\Omega}} \Delta \tilde{\mathbf{r}}^{-1} \mathbf{j}_{h}^{\|}\left(\tilde{\mathbf{r}}^{\prime}\right) d \tilde{V}^{\prime}, \quad \forall \tilde{\mathbf{r}} \in \tilde{\Omega} .
\end{aligned}
$$

Since $\chi_{h}^{\|}$is an eigenvalue of the left-hand side of Eq. (D9) (in the space of longitudinal function), according to the normal solvability condition [61,62] a solution of Eq. (D9) exists only under the condition that its right-hand side is orthogonal to the eigencurrent $\mathbf{j}_{h}^{\|}$:

$$
\left\|\mathbf{j}_{h}^{\|}\right\|^{2} \frac{\chi_{h}^{(2)}}{\chi_{h}^{\|}}+\frac{\chi_{h}^{\|}}{4 \pi} \int_{\tilde{\Omega}} \mathbf{j}_{h}^{\|}(\tilde{\mathbf{r}}) \cdot \tilde{\nabla} \oint_{\partial \tilde{\Omega}} \frac{\Delta \tilde{\mathbf{r}}}{2 !} j_{n, h}^{\|}\left(\tilde{\mathbf{r}}^{\prime}\right) d \tilde{S}^{\prime} d \tilde{V}+\frac{\chi_{h}^{\|}}{4 \pi} \int_{\tilde{\Omega}} \mathbf{j}_{h}^{\|}(\tilde{\mathbf{r}}) \cdot \int_{\tilde{\Omega}} \Delta \tilde{\mathbf{r}}^{-1} \mathbf{j}_{h}^{\|}\left(\tilde{\mathbf{r}}^{\prime}\right) d \tilde{V}^{\prime} d \tilde{V}=0
$$

Using the divergence theorem in the above equation, it is found that the Eq. (D9) is solvable when

$$
\chi_{h}^{(2)}=-\frac{\left(\chi_{h}^{\|}\right)^{2}}{\left\|\mathbf{j}_{h}^{\|}\right\|^{2}} \frac{1}{4 \pi}\left(\oint_{\partial \tilde{\Omega}} j_{n, h}^{\|}(\tilde{\mathbf{r}}) \oint_{\partial \tilde{\Omega}} \frac{\left|\tilde{\mathbf{r}}-\tilde{\mathbf{r}}^{\prime}\right|}{2} j_{n, h}^{\|}\left(\tilde{\mathbf{r}}^{\prime}\right) d \tilde{S}^{\prime} d \tilde{S}+\int_{\tilde{\Omega}} \mathbf{j}_{h}^{\|}(\tilde{\mathbf{r}}) \cdot \int_{\tilde{\Omega}} \frac{\mathbf{j}_{h}^{\|}\left(\tilde{\mathbf{r}}^{\prime}\right)}{\left|\tilde{\mathbf{r}}-\tilde{\mathbf{r}}^{\prime}\right|} d \tilde{V}^{\prime} d \tilde{V}\right) .
$$


The second-order radiative correction $\chi_{h}^{(2)}$ results from two contributions: The first term in parentheses in Eq. (D11) is associated to the radiative self-interaction of the surface charges of the plasmon mode $\mathbf{j}_{h}^{\|}$through the scalar potential. The second term is instead proportional to the magnetostatic energy of the current mode $\mathbf{j}_{h}^{\|}$:

$$
\mathscr{W}_{m}\left\{\mathbf{j}_{h}^{\|}\right\}=\frac{1}{2} \int_{\tilde{\Omega}} \mathbf{j}_{h}^{\|} \cdot \mathbf{A}\left\{\mathbf{j}_{h}^{\|}\right\} d \tilde{V}=\frac{\mu_{0}}{8 \pi} \int_{\tilde{\Omega}} \mathbf{j}_{h}^{\|}(\tilde{\mathbf{r}}) \cdot \int_{\tilde{\Omega}} \frac{\mathbf{j}_{h}^{\|}\left(\tilde{\mathbf{r}}^{\prime}\right)}{\left|\tilde{\mathbf{r}}-\tilde{\mathbf{r}}^{\prime}\right|} d \tilde{V}^{\prime} d \tilde{V},
$$

where $\mathbf{A}\left\{\mathbf{j}_{h}^{\|}\right\}$is the vector potential generated by the current $\mathbf{j}_{h}^{\|}$in free space. The second-order correction to the EQS mode has both longitudinal and transverse components, called $\mathbf{u}_{h}^{(2) \|}$ and $\mathbf{u}_{h}^{(2) \perp}$, respectively:

$$
\mathbf{u}_{h}^{(2)}(\tilde{\mathbf{r}})=\mathbf{u}_{h}^{(2) \|}(\tilde{\mathbf{r}})+\mathbf{u}_{h}^{(2) \perp}(\tilde{\mathbf{r}}), \quad \forall \tilde{\mathbf{r}} \in \tilde{\Omega} .
$$

The longitudinal part $\mathbf{u}_{h}^{(2) \|}$ belongs to the space of longitudinal functions (div- and curl-free functions within $\Omega$ which have nonzero normal component on $\partial \tilde{\Omega}$ ). It can be represented in terms of the basis of EQS current modes $\left\{\mathbf{j}_{k}^{\|}\right\}_{k \in \mathbb{N}}$

$$
\mathbf{u}_{h}^{(2) \|}=\sum_{k=1}^{\infty} \alpha_{h, k}^{(2)} \mathbf{j}_{k}^{\|} .
$$

By using Eq. (D14) into Eq. (D9), and solving the resulting equation in the weak from by testing along the element of the basis $\left\{\mathbf{j}_{k}^{\|}\right\}_{k \in \mathbb{N}}$, it is obtained that $\alpha_{h, h}^{(2)}=0$ and

$$
\alpha_{h, k}^{(2)}=\frac{1}{4 \pi} \frac{1}{\left\|\mathbf{j}_{k}^{\|}\right\|^{2}} \frac{\chi_{k}^{\|} \chi_{h}^{\|}}{\chi_{k}^{\|}-\chi_{h}^{\|}}\left(\oint_{\partial \tilde{\Omega}} j_{n, h}^{\|}(\tilde{\mathbf{r}}) \oint_{\partial \tilde{\Omega}} \frac{\left|\tilde{\mathbf{r}}-\tilde{\mathbf{r}}^{\prime}\right|}{2} j_{n, k}^{\|}\left(\tilde{\mathbf{r}}^{\prime}\right) d \tilde{S}^{\prime} d \tilde{S}+\int_{\tilde{\Omega}} \mathbf{j}_{h}^{\|}(\tilde{\mathbf{r}}) \cdot \int_{\tilde{\Omega}} \frac{\mathbf{j}_{k}^{\|}\left(\tilde{\mathbf{r}}^{\prime}\right)}{\left|\tilde{\mathbf{r}}-\tilde{\mathbf{r}}^{\prime}\right|} d \tilde{V}^{\prime} d \tilde{V}\right), \forall k \neq h .
$$

The transverse part $\mathbf{u}_{h}^{(2) \perp}$ belongs to the space of transverse functions (div-free functions within $\tilde{\Omega}$ and with zero normal component on $\partial \tilde{\Omega})$, and can be represented in terms of the basis of the magnetoquasistatic current modes $\left\{\mathbf{j}_{k}^{\perp}\right\}_{k \in \mathbb{N}}$

$$
\mathbf{u}_{h}^{(2) \perp}=\sum_{k=1}^{\infty} \beta_{h, k}^{(2)} \mathbf{j}_{k}^{\perp} .
$$

By substituting the expansion (D16) in Eq. (D9) and testing the resulting equation in the transverse vector space, spanned by the MQS current modes $\left\{\mathbf{j}_{k}^{\perp}\right\}_{k \in \mathbb{N}}$, the expansion coefficients are found:

$$
\beta_{h, k}^{(2)}=\frac{1}{4 \pi} \frac{\chi_{h}^{\|}}{\left\|\mathbf{j}_{k}^{\perp}\right\|^{2}} \int_{\tilde{\Omega}} \mathbf{j}_{h}^{\|}(\tilde{\mathbf{r}}) \cdot \int_{\tilde{\Omega}} \frac{\mathbf{j}_{k}^{\perp}\left(\tilde{\mathbf{r}}^{\prime}\right)}{\left|\tilde{\mathbf{r}}-\tilde{\mathbf{r}}^{\prime}\right|} d \tilde{V}^{\prime} d \tilde{V} \quad \forall k \in \mathbb{N} .
$$

\section{Third order}

Matching the third-order terms in Eq. (26), the following integrodifferential equation for the third-order correction $\mathbf{u}_{h}^{(3)}$ is obtained

$$
\begin{aligned}
\chi_{h}^{\|} & \tilde{\nabla} \oint_{\partial \tilde{\Omega}} \Delta \tilde{\mathbf{r}}^{-1} u_{n, h}^{(3)}\left(\tilde{\mathbf{r}}^{\prime}\right) d \tilde{S}^{\prime}+4 \pi \mathbf{u}_{h}^{(3)}(\tilde{\mathbf{r}}) \\
& =-\chi_{h}^{(3)} \tilde{\nabla} \oint_{\partial \tilde{\Omega}} \Delta \tilde{\mathbf{r}}^{-1} j_{n, h}^{\|}\left(\tilde{\mathbf{r}}^{\prime}\right) d \tilde{S}^{\prime}-i \chi_{h}^{\|} \tilde{\nabla} \oint_{\partial \tilde{\Omega}} \frac{\Delta \tilde{\mathbf{r}}^{2}}{3 !} j_{n, h}^{\|}\left(\tilde{\mathbf{r}}^{\prime}\right) d \tilde{S}^{\prime}-i \chi_{h}^{\|} \int_{\tilde{\Omega}} \mathbf{j}_{h}^{\|}\left(\tilde{\mathbf{r}}^{\prime}\right) d \tilde{V}^{\prime} \quad \forall \tilde{\mathbf{r}} \in \tilde{\Omega} .
\end{aligned}
$$

Because of the normal solvability condition [61,62], a solution of Eq. (D18) (in the weak form in the space of longitudinal functions) exists only if its right-hand side is orthogonal to the EQS mode $\mathbf{j}_{h}^{\|}$:

$$
-4 \pi \frac{\chi_{h}^{(3)}}{\chi_{h}^{\|}}\left\|\mathbf{j}_{h}^{\|}\right\|^{2}+i \chi_{h}^{\|} \int_{\tilde{\Omega}} \mathbf{j}_{h}^{\|}(\tilde{\mathbf{r}}) \cdot \tilde{\nabla} \oint_{\partial \tilde{\Omega}} \frac{\Delta \tilde{\mathbf{r}}^{2}}{3 !} j_{n, h}^{\|}\left(\tilde{\mathbf{r}}^{\prime}\right) d \tilde{S}^{\prime} d \tilde{V}+i \chi_{h}^{\|} \int_{\tilde{\Omega}} \mathbf{j}_{h}^{\|}(\tilde{\mathbf{r}}) d \tilde{V} \cdot \int_{\tilde{\Omega}} \mathbf{j}_{h}^{\|}\left(\tilde{\mathbf{r}}^{\prime}\right) d \tilde{V}^{\prime}=0,
$$

where the fact that $\mathbf{j}_{h}^{\|}$is an eigenvector of the EQS operator is used. Thus, the third-order correction $\chi_{h}^{(3)}$ is immediately obtained:

$$
\chi_{h}^{(3)}=i \frac{\left(\chi_{h}^{\|}\right)^{2}}{\left\|\mathbf{j}_{h}^{\|}\right\|^{2}} \frac{1}{4 \pi}\left(\oint_{\partial \tilde{\Omega}} j_{n, h}^{\|}(\tilde{\mathbf{r}}) \oint_{\partial \tilde{\Omega}} \frac{\Delta \tilde{\mathbf{r}}^{2}}{3 !} j_{n, h}^{\|}\left(\tilde{\mathbf{r}}^{\prime}\right) d \tilde{S}^{\prime} d \tilde{S}+\int_{\tilde{\Omega}} \mathbf{j}_{h}^{\|}(\tilde{\mathbf{r}}) d \tilde{V} \cdot \int_{\tilde{\Omega}} \mathbf{j}_{h}^{\|}\left(\tilde{\mathbf{r}}^{\prime}\right) d \tilde{V}^{\prime}\right) .
$$

The above expression can be further manipulated, and eventually rewritten in terms of the multipolar components of the quasistatic mode. By using the fact that $\Delta \tilde{\mathbf{r}}^{2}=\left|\tilde{\mathbf{r}}-\tilde{\mathbf{r}}^{\prime}\right|^{2}=\left(\tilde{\mathbf{r}}-\tilde{\mathbf{r}}^{\prime}\right) \cdot\left(\tilde{\mathbf{r}}-\tilde{\mathbf{r}}^{\prime}\right)=\|\tilde{\mathbf{r}}\|^{2}+\left\|\tilde{\mathbf{r}}^{\prime}\right\|^{2}-2 \tilde{\mathbf{r}} \cdot \tilde{\mathbf{r}}^{\prime}$, the first term in parentheses 
of Eq. (D20) is recast as follows:

$$
\begin{aligned}
\oint_{\partial \tilde{\Omega}} j_{n, h}^{\|}(\tilde{\mathbf{r}}) & \oint_{\partial \tilde{\Omega}} \frac{\Delta \tilde{\mathbf{r}}^{2}}{3 !} j_{n, h}^{\|}\left(\tilde{\mathbf{r}}^{\prime}\right) d \tilde{S} d \tilde{S}^{\prime}=\left(\oint_{\partial \tilde{\Omega}} j_{n, h}^{\|}(\tilde{\mathbf{r}}) d \tilde{S}\right) \cdot\left(\oint_{\partial \tilde{\Omega}} \frac{\left\|\tilde{\mathbf{r}}^{\prime}\right\|^{2}}{3 !} j_{n, h}^{\|}\left(\tilde{\mathbf{r}}^{\prime}\right) d \tilde{S}^{\prime}\right) \\
& +\left(\oint_{\partial \tilde{\Omega}} j_{n, h}^{\|}\left(\tilde{\mathbf{r}}^{\prime}\right) d \tilde{S}^{\prime}\right) \cdot\left(\oint_{\partial \tilde{\Omega}} \frac{\|\tilde{\mathbf{r}}\|^{2}}{3 !} j_{n, h}^{\|}(\tilde{\mathbf{r}}) d \tilde{S}\right)-\frac{1}{3}\left(\oint_{\tilde{\Omega}} \tilde{\mathbf{r}} J_{n, h}(\tilde{\mathbf{r}}) d \tilde{S}\right) \cdot\left(\oint_{\partial \tilde{\Omega}} \tilde{\mathbf{r}}^{\prime} J_{n, h}\left(\tilde{\mathbf{r}}^{\prime}\right) d \tilde{S}^{\prime}\right)=-\frac{1}{3}\left\|\mathbf{P}_{\mathrm{E} \mid h}^{\|}\right\|^{2},
\end{aligned}
$$

where the definition of electric dipole moment [Eq. (A1) in Appendix A] and the charge neutrality condition were used. The second term in parentheses of Eq. (D20), by using the definition of electric dipole moment, can be written as

$$
\left(\int_{\tilde{\Omega}} \mathbf{j}_{h}^{\|}\left(\tilde{\mathbf{r}}^{\prime}\right) d \tilde{V}\right) \cdot\left(\int_{\tilde{\Omega}} \mathbf{j}_{h}^{\|}\left(\tilde{\mathbf{r}}^{\prime}\right) d \tilde{V}^{\prime}\right)=\left\|\mathbf{P}_{\mathrm{E} \mid h}^{\|}\right\|^{2} .
$$

Eventually, by using Eqs. (D21) and (D22) into Eq. (D20), the third-order correction is expressed in terms of the electric dipole moment of the EQS mode:

$$
\chi_{h}^{(3)}=i \frac{1}{6 \pi} \frac{\left(\chi_{h}^{\|}\right)^{2}}{\left\|\mathbf{j}_{h}^{\|}\right\|^{2}}\left\|\mathbf{P}_{\mathrm{E} \mid h}^{\|}\right\|^{2}
$$

The third-order correction of the current density mode is entirely longitudinal, i.e., $\mathbf{u}_{h}^{(3) \perp}=0$ :

$$
\mathbf{u}_{h}^{(3)}=\mathbf{u}_{h}^{(3) \|} .
$$

This fact can be directly obtained from Eq. (D18), using the property that transverse function has zero dipole moment. The current density $\mathbf{u}_{h}^{(3) \|}$ belongs to the space of longitudinal functions. Thus, it can be represented in terms of the basis of the EQS current modes $\left\{\mathbf{j}_{k}^{\|}\right\}_{k \in \mathbb{N}}$

$$
\mathbf{u}_{h}^{(3) \|}=\sum_{k=1}^{\infty} \alpha_{h, k}^{(3)} \mathbf{j}_{k}^{\|}
$$

By substituting Eq. (D25) into Eq. (D18), we obtain $\alpha_{h, h}^{(3)}=0 \forall h \in \mathbb{N}$ and

$$
\alpha_{h, k}^{(3)}=i \frac{\chi_{h}^{\|} \chi_{k}^{\|}}{\chi_{h}^{\|}-\chi_{k}^{\|}} \frac{1}{4 \pi}\left(\oint_{\partial \tilde{\Omega}} j_{n, k}^{\|}(\tilde{\mathbf{r}}) \oint_{\partial \tilde{\Omega}} \frac{\left|\tilde{\mathbf{r}}-\tilde{\mathbf{r}}^{\prime}\right|^{2}}{3 !} j_{n, h}^{\|}\left(\tilde{\mathbf{r}}^{\prime}\right) d \tilde{S}^{\prime} d \tilde{S}+\int_{\tilde{\Omega}} \mathbf{j}_{k}^{\|}(\tilde{\mathbf{r}}) d \tilde{V} \cdot \int_{\tilde{\Omega}} \mathbf{j}_{h}^{\|}\left(\tilde{\mathbf{r}}^{\prime}\right) d \tilde{V}^{\prime}\right) \quad \forall h \neq k .
$$

By using the same arguments used in the derivation of Eqs. (D21) and (D22), it is immediately seen that (D26) can be also written as

$$
\alpha_{h, k}^{(3)}=i \frac{1}{6 \pi} \frac{1}{\left\|\mathbf{j}_{k}^{\|}\right\|^{2}} \frac{\chi_{h}^{\|} \chi_{k}^{\|}}{\chi_{h}^{\|}-\chi_{k}^{\|}}\left(\mathbf{P}_{\mathrm{E}, h}^{\|} \cdot \mathbf{P}_{\mathrm{E}, k}^{\|}\right) \quad \forall h \neq k
$$

\section{Fifth order}

As shown in the main paper, dark plasmonic modes exhibit vanishing third-order correction $\chi_{h}^{(3)}$. In this case, to retrieve information about the radiative Q-factor, it is mandatory to consider the fifth-order perturbation $\chi_{h}^{(5)}$. By collecting the fifth-order terms in Eq. (26), the following integrodifferential equation for the fifth-order correction $\mathbf{u}_{h}^{(5)}$ is obtained:

$$
\begin{aligned}
& 4 \pi \mathbf{u}_{h}^{(5)}(\tilde{\mathbf{r}})+\chi_{h}^{\|} \tilde{\nabla} \oint_{\partial \tilde{\Omega}} \Delta \tilde{\mathbf{r}}^{-1} u_{n, h}^{(5)}\left(\tilde{\mathbf{r}}^{\prime}\right) d \tilde{S}^{\prime}=+i \chi_{h}^{\|} \tilde{\nabla} \oint_{\partial \tilde{\Omega}} \frac{\Delta \tilde{\mathbf{r}}^{4}}{5 !} j_{n, h}^{\|}\left(\tilde{\mathbf{r}}^{\prime}\right) d \tilde{S}^{\prime}-\chi_{h}^{(2)} \tilde{\nabla} \oint_{\partial \tilde{\Omega}} \Delta \tilde{\mathbf{r}}^{-1} u_{n, h}^{(3)}\left(\tilde{\mathbf{r}}^{\prime}\right) d \tilde{S}^{\prime} \\
& \quad-i \chi_{h}^{(2)} \tilde{\nabla} \oint_{\partial \tilde{\Omega}} \frac{\Delta \tilde{\mathbf{r}}^{2}}{3 !} j_{n, h}^{\|}\left(\tilde{\mathbf{r}}^{\prime}\right) d \tilde{S}^{\prime}+\chi_{h}^{(3)} \tilde{\nabla} \oint_{\partial \tilde{\Omega}} \frac{\Delta \tilde{\mathbf{r}}}{2} j_{n, h}^{\|}\left(\tilde{\mathbf{r}}^{\prime}\right) d \tilde{S}^{\prime}-\chi_{h}^{(5)} \tilde{\nabla} \oint_{\partial \tilde{\Omega}} \Delta \tilde{\mathbf{r}}^{-1} j_{n, h}^{\|}\left(\tilde{\mathbf{r}}^{\prime}\right) d \tilde{S}^{\prime} \\
& \quad+\chi_{h}^{\|} \int_{\tilde{\Omega}} \Delta \tilde{\mathbf{r}}^{-1} \mathbf{u}_{h}^{(3)}(\tilde{\mathbf{r}}) d \tilde{V}^{\prime}+i \chi_{h}^{\|} \int_{\tilde{\Omega}} \frac{\Delta \tilde{\mathbf{r}}^{2}}{3 !} \mathbf{j}_{h}^{\|}(\tilde{\mathbf{r}}) d \tilde{V}^{\prime}-i \chi_{h}^{\|} \int_{\tilde{\Omega}} \mathbf{u}_{h}^{(2)}(\tilde{\mathbf{r}}) d \tilde{V}^{\prime}, \quad \forall \tilde{\mathbf{r}} \in \tilde{\Omega} .
\end{aligned}
$$

Because of the normal solvability condition [61,62], a solution of Eq. (D28) (in the weak form in the space of longitudinal functions) exists only under the condition that its right-hand side is orthogonal to the current mode $\mathbf{j}_{h}^{\|}$, namely when the following 
condition is verified:

$$
\begin{aligned}
\chi_{h}^{(5)}= & -\frac{1}{4 \pi} \frac{\chi_{h}^{\|}}{\left\|\mathbf{j}_{h}^{\|}\right\|^{2}}\left[i \chi_{h}^{\|} \oint_{\partial \tilde{\Omega}} j_{n, h}^{\|}(\tilde{\mathbf{r}}) \oint_{\partial \tilde{\Omega}} \frac{\Delta \tilde{\mathbf{r}}^{4}}{5 !} j_{n, h}^{\|}\left(\tilde{\mathbf{r}}^{\prime}\right) d \tilde{S}^{\prime} d \tilde{V}-\chi_{h}^{(2)} \oint_{\partial \tilde{\Omega}} j_{n, h}^{\|}(\tilde{\mathbf{r}}) \oint_{\partial \tilde{\Omega}} \Delta \tilde{\mathbf{r}}^{-1} u_{n, h}^{(3)}\left(\tilde{\mathbf{r}}^{\prime}\right) d \tilde{S}^{\prime} d \tilde{V}\right. \\
& -i \chi_{h}^{(2)} \oint_{\partial \tilde{\Omega}} j_{n, h}^{\|}(\tilde{\mathbf{r}}) \oint_{\partial \tilde{\Omega}} \frac{\Delta \tilde{\mathbf{r}}^{2}}{3 !} j_{n, h}^{\|}\left(\tilde{\mathbf{r}}^{\prime}\right) d \tilde{S}^{\prime} d \tilde{V}+\chi_{h}^{(3)} \oint_{\partial \tilde{\Omega}} j_{n, h}^{\|}(\tilde{\mathbf{r}}) \oint_{\partial \tilde{\Omega}} \frac{\Delta \tilde{\mathbf{r}}}{2} j_{n, h}^{\|}\left(\tilde{\mathbf{r}}^{\prime}\right) d \tilde{S}^{\prime} d \tilde{V} \\
& +\chi_{h}^{\|} \int_{\tilde{\Omega}} \mathbf{j}_{h}^{\|}(\tilde{\mathbf{r}}) \cdot \int_{\tilde{\Omega}} \Delta \tilde{\mathbf{r}}^{-1} \mathbf{u}_{h}^{(3)}\left(\tilde{\mathbf{r}}^{\prime}\right) d \tilde{V}^{\prime} d \tilde{V}+i \chi_{h}^{\|} \int_{\tilde{\Omega}} \mathbf{j}_{h}^{\|}(\tilde{\mathbf{r}}) \cdot \int_{\tilde{\Omega}} \frac{\Delta \tilde{\mathbf{r}}^{2}}{3 !} \mathbf{j}_{h}^{\|}\left(\tilde{\mathbf{r}}^{\prime}\right) d \tilde{V}^{\prime} d \tilde{V}-i \chi_{h}^{\|}\left(\int_{\tilde{\Omega}} \mathbf{j}_{h}^{\|}(\tilde{\mathbf{r}}) d \tilde{V}\right) \\
& \left.\cdot\left(\int_{\tilde{\Omega}} \mathbf{u}_{h}^{(2)}\left(\tilde{\mathbf{r}}^{\prime}\right) d \tilde{V}^{\prime}\right)\right] .
\end{aligned}
$$

By assuming that the $h$ th EQS mode is dark, namely that the dipole moment of the $h$ th EQS mode $\mathbf{P}_{\mathrm{E}, h}^{\|}$is zero, the third-order corrections $\chi_{h}^{(3)}=0$ and $u_{n, h}^{(3)}=\mathbf{0}$ vanish. The integrals below also vanish for the same reason:

$$
\begin{aligned}
& \int_{\tilde{\Omega}} \mathbf{j}_{h}^{\|}(\tilde{\mathbf{r}}) d \tilde{V}=\mathbf{P}_{\mathrm{E}, h}=\mathbf{0}, \\
& \int_{\tilde{\Omega}} \mathbf{j}_{h}^{\|}(\tilde{\mathbf{r}}) \cdot \tilde{\nabla} \oint_{\partial \tilde{\Omega}} \Delta \tilde{\mathbf{r}}^{2} j_{n, h}^{\|}\left(\tilde{\mathbf{r}}^{\prime}\right) d \tilde{S}^{\prime} d \tilde{V}=2\left\|\mathbf{P}_{\mathrm{E}, h}^{\|}\right\|^{2}=0 .
\end{aligned}
$$

Thus, Eq. (D29) becomes

$$
\chi_{h}^{(5)}=-i \frac{\left(\chi_{h}^{\|}\right)^{2}}{\left\|\mathbf{j}_{h}^{\|}\right\|^{2}} \frac{1}{4 \pi}\left[\oint_{\partial \tilde{\Omega}} j_{n, h}^{\|}(\tilde{\mathbf{r}}) \cdot \oint_{\partial \tilde{\Omega}} \frac{\Delta \tilde{\mathbf{r}}^{4}}{5 !} j_{n, h}^{\|}\left(\tilde{\mathbf{r}}^{\prime}\right) d S^{\prime} d \tilde{S}+\int_{\tilde{\Omega}} \mathbf{j}_{h}^{\|}(\tilde{\mathbf{r}}) \cdot \int_{\tilde{\Omega}} \frac{\Delta \tilde{\mathbf{r}}^{2}}{3 !} \mathbf{j}_{h}^{\|}\left(\tilde{\mathbf{r}}^{\prime}\right) d \tilde{V}^{\prime} d \tilde{V}\right] .
$$

The above expression can be further manipulated and eventually rewritten in terms of the multipolar components of the quasistatic mode, specifically in terms of the electric quadrupole moment of the EQS mode $\mathbf{j}_{h}^{\|}$, called $\overleftrightarrow{\mathbf{Q}}_{\mathrm{E} \mid h}^{\|}$, whose components are denoted as $Q_{\mathrm{E}|h| i j}^{\|}$. The electric quadrupole is defined by Eq. (A2) in Appendix A. For dark EQS modes, the following identities holds:

$$
\begin{gathered}
\oint_{\partial \tilde{\Omega}} j_{n, h}^{\|}(\tilde{\mathbf{r}}) \cdot \oint_{\partial \tilde{\Omega}} \frac{\left|\tilde{\mathbf{r}}-\tilde{\mathbf{r}}^{\prime}\right|^{4}}{5 !} j_{n, h}^{\|}\left(\tilde{\mathbf{r}}^{\prime}\right) d S^{\prime} d \tilde{S}=\frac{1}{60}\left[2 \sum_{i j} Q_{\mathrm{E}|h| i j}^{\|}+\left(\operatorname{Tr} \overleftrightarrow{\mathbf{Q}}_{\mathrm{E} \mid h}^{\|}\right)^{2}\right], \\
\int_{\tilde{\Omega}} \mathbf{j}_{h}^{\|}(\tilde{\mathbf{r}}) \cdot \int_{\tilde{\Omega}} \frac{\left|\tilde{\mathbf{r}}-\tilde{\mathbf{r}}^{\prime}\right|^{2}}{3 !} \mathbf{j}_{h}^{\|}\left(\tilde{\mathbf{r}}^{\prime}\right) d \tilde{V}^{\prime} d \tilde{V}=-\frac{1}{12} \sum_{i j} Q_{\mathrm{E}|h| i j}^{\|},
\end{gathered}
$$

where $\mathrm{Tr}$ is the trace operator. By using Eqs. (D32) and (D33) in Eq. (D31), the fifth-order correction is eventually expressed in terms of the power radiated to infinity of the electric quadrupole component $\overleftrightarrow{\mathbf{Q}}_{\mathrm{E} \mid h}^{\|}$of the $h$ th EQS mode:

$$
\chi_{h}^{(5)}=i \frac{1}{80 \pi} \frac{\left(\chi_{h}^{\|}\right)^{2}}{\left\|\mathbf{j}_{h}^{\|}\right\|^{2}}\left[\sum_{i j} Q_{\mathrm{E}|h| i j}^{\|}-\frac{1}{3}\left(\operatorname{Tr} \overleftrightarrow{\mathbf{Q}}_{\mathrm{E} \mid h}^{\|}\right)^{2}\right] .
$$

\section{APPENDIX E: DERIVATION OF RADIATION CORRECTIONS FOR DIELECTRIC MODES AND RESONANCES}

In this appendix the derivation of the radiation corrections for the dielectric modes and resonances is carried out in detail, by matching same order terms in Eq. (38).

\section{1. zero order}

Matching the zeroth-order terms of Eq. (38), the following equation is obtained:

$$
\kappa_{h}^{(0)} \tilde{\nabla} \oint_{\partial \tilde{\Omega}} \Delta \tilde{\mathbf{r}}^{-1} v_{h n}^{(0)}\left(\tilde{\mathbf{r}}^{\prime}\right) d \tilde{S}^{\prime}=0, \quad \forall \tilde{\mathbf{r}} \in \tilde{\Omega} .
$$

Therefore, assuming $\kappa_{h}^{(0)} \neq 0, v_{h n}^{(0)}$ vanishes on $\partial \Omega$, namely,

$$
v_{h n}^{(0)}(\tilde{\mathbf{r}})=0, \quad \forall \tilde{\mathbf{r}} \in \partial \tilde{\Omega} .
$$

Thus, $\mathbf{v}_{h}^{(0)}$ is a purely transverse vector field. 


\section{First order}

Matching the first-order terms in Eq. (38) and by also considering Eq. (E2), the following equation is obtained:

$$
\kappa_{h}^{(0)} \tilde{\nabla} \oint_{\partial \tilde{\Omega}} \Delta \tilde{\mathbf{r}}^{-1} v_{h n}^{(1)}\left(\tilde{\mathbf{r}}^{\prime}\right) d \tilde{S}^{\prime}=0, \quad \forall \tilde{\mathbf{r}} \in \tilde{\Omega}
$$

Therefore, also the normal component to $\partial \Omega$ of the first-order correction to any MQS current mode vanishes on $\partial \Omega$, namely,

$$
v_{h n}^{(1)}(\tilde{\mathbf{r}})=0 \quad \forall \tilde{\mathbf{r}} \in \partial \tilde{\Omega} .
$$

\section{Second order}

Collecting the second-order terms in Eq. (38), the following integrodifferential equation is obtained:

$$
-4 \pi \mathbf{v}_{h}^{(0)}(\tilde{\mathbf{r}})-\kappa_{h}^{(0)} \tilde{\nabla} \oint_{\partial \tilde{\Omega}} \Delta \tilde{\mathbf{r}}^{-1} v_{h n}^{(2)}\left(\tilde{\mathbf{r}}^{\prime}\right) d \tilde{S}^{\prime}+\kappa_{h}^{(0)} \int_{\tilde{\Omega}} \Delta \tilde{\mathbf{r}}^{-1} \mathbf{v}_{h}^{(0)}\left(\tilde{\mathbf{r}}^{\prime}\right) d \tilde{V}^{\prime}, \quad \forall \tilde{\mathbf{r}} \in \tilde{\Omega} .
$$

By enforcing Eq. (E5) in the weak form in the space of transverse functions (div-free functions in $\tilde{\Omega}$, which also exhibit vanishing normal component to $\partial \tilde{\Omega}$ ), the magnetoquasistatic (MQS) problem introduced in Eq. (8) of the main paper is obtained. Thus,

$$
\begin{gathered}
\kappa_{h}^{(0)}=\kappa_{h}^{\perp}, \\
\mathbf{v}_{h}^{(0)}(\tilde{\mathbf{r}})=\mathbf{j}_{h}^{\perp}(\tilde{\mathbf{r}}), \quad \forall \tilde{\mathbf{r}} \in \tilde{\Omega},
\end{gathered}
$$

where $\kappa_{h}^{\perp}$ and $\mathbf{j}_{h}^{\perp}$ are the MQS eigenvalue and the MQS current modes, respectively. However, by testing the same equation, (E5), in the space of longitudinal functions (div-free and curl-free function in $\tilde{\Omega}$ but with a nonvanishing normal component to $\partial \tilde{\Omega})$, the longitudinal component of the second-order mode perturbation $\mathbf{v}_{h}^{(2)}$, called $v_{h n}^{(2)}$, can be calculated. The derivation of $v_{h n}^{(2)}$ is straightforward, if $\mathbf{v}_{h}^{(2) \|}$ is expanded in terms of the EQS (longitudinal) current modes:

$$
\mathbf{v}_{h}^{(2) \|}=\sum_{k=1}^{\infty} \alpha_{h, k}^{(2)} \mathbf{j}_{k}^{\|} .
$$

By substituting (E8) in Eq. (E5), and by projecting the resulting expression in the space of longitudinal functions, which is spanned by the set of EQS modes $\left\{\mathbf{j}_{h}^{\|}\right\}_{h \in \mathbb{N}}$, the expansion coefficients $\alpha_{h, k}^{(2)}$ are determined:

$$
\alpha_{h, k}^{(2)}=-\frac{\chi_{k}^{\|}}{4 \pi} \frac{1}{\left\|\mathbf{j}_{k}^{\|}\right\|^{2}} \int_{\tilde{\Omega}} \mathbf{j}_{k}^{\|}(\tilde{\mathbf{r}}) \cdot \int_{\tilde{\Omega}} \frac{\mathbf{j}_{h}^{\perp}\left(\tilde{\mathbf{r}}^{\prime}\right)}{\left|\tilde{\mathbf{r}}-\tilde{\mathbf{r}}^{\prime}\right|} d \tilde{V}^{\prime} d \tilde{V} .
$$

Each coefficient $\alpha_{h, k}^{(2)}$ is proportional to the magnetostatic interaction energy between the MQS current mode $\mathbf{j}_{h}^{\perp}$ and the EQS current modes $\mathbf{j}_{k}^{\|}$, denoted as $\mathscr{W}_{m \mathrm{I}}\left\{\mathbf{j}_{k}^{\|}, \mathbf{j}_{h}^{\perp}\right\}$ :

$$
\mathscr{W}_{m \mathrm{I}}\left\{\mathbf{j}_{k}^{\|}, \mathbf{j}_{h}^{\perp}\right\}=\frac{1}{2} \int_{\tilde{\Omega}} \mathbf{j}_{k}^{\|} \cdot \mathbf{A}\left\{\mathbf{j}_{h}^{\perp}\right\} d \tilde{V}=\frac{\mu_{0}}{8 \pi} \int_{\tilde{\Omega}} \mathbf{j}_{k}^{\|}(\tilde{\mathbf{r}}) \cdot \int_{\tilde{\Omega}} \frac{\mathbf{j}_{h}^{\perp}\left(\tilde{\mathbf{r}}^{\prime}\right)}{\left|\tilde{\mathbf{r}}-\tilde{\mathbf{r}}^{\prime}\right|} d \tilde{V}^{\prime} d \tilde{V},
$$

where $\mathbf{A}\left\{\mathbf{j}_{h}^{\perp}\right\}$ is the vector potential generated by the current mode $\mathbf{j}_{h}^{\perp}$. If the $h$ th MQS current mode generates a purely transverse vector potential, i.e. it is a $\mathbb{A}^{\perp}$ mode accordingly to definition (15), the interaction energy between it and any EQS mode is vanishing.

We recall that, accordingly to Eq. (11) of the main paper, any magnetoquasistatic mode has a zero electric dipole moment. Nevertheless, accordingly to Eq. (E8), its second-order radiative correction $\mathbf{v}_{h}^{(2)}$ may exhibit a nonzero electric dipole moment, called $\mathbf{P}_{\mathrm{E} \mid h}^{(2)}$. It is given by

$$
\mathbf{P}_{\mathrm{E} \mid h}^{(2)}=\sum_{k=1}^{\infty} \alpha_{h, k}^{(2)} \mathbf{P}_{\mathrm{E} \mid k}^{\|},
$$

where $\mathbf{P}_{\mathrm{E} \mid k}^{\|}$is the electric dipole moment of the $k$ th EQS mode $\mathbf{j}_{k}^{\|}$. For $\mathbb{A}^{\perp}$ modes, the longitudinal part of $\mathbf{v}_{h}^{(2)}$ vanishes, and also $\mathbf{P}_{\mathrm{E} \mid h}^{(2)}$ vanishes.

\section{Third order}

Matching the third-order terms of Eq. (38), the following equation is obtained:

$$
\begin{aligned}
4 \pi \mathbf{v}_{h}^{(1)}(\tilde{\mathbf{r}})-\kappa_{h}^{\perp} \int_{\tilde{\Omega}} \Delta \tilde{\mathbf{r}}^{-1} \mathbf{v}_{h}^{(1) \perp}\left(\tilde{\mathbf{r}}^{\prime}\right) d \tilde{V}^{\prime} & =-\kappa_{h}^{\perp} \tilde{\nabla} \oint_{\partial \tilde{\Omega}} \Delta \tilde{\mathbf{r}}^{-1} v_{h n}^{(3)}\left(\tilde{\mathbf{r}}^{\prime}\right) d \tilde{S}^{\prime}-\kappa_{h}^{(1)} \tilde{\nabla} \oint_{\partial \tilde{\Omega}} \Delta \tilde{\mathbf{r}}^{-1} v_{h n}^{(2)}\left(\tilde{\mathbf{r}}^{\prime}\right) d \tilde{S}^{\prime} \\
& +i \kappa_{h}^{\perp} \tilde{\nabla} \oint_{\partial \tilde{\Omega}} v_{h n}^{(2)}\left(\tilde{\mathbf{r}}^{\prime}\right) d \tilde{S}^{\prime}+\kappa_{h}^{(1)} \int_{\tilde{\Omega}} \Delta \tilde{\mathbf{r}}^{-1} \mathbf{j}_{h}^{\perp}\left(\tilde{\mathbf{r}}^{\prime}\right) d \tilde{V}^{\prime}-i x \kappa_{h}^{\perp} \int_{\tilde{\Omega}} \mathbf{j}_{h}^{\perp}\left(\tilde{\mathbf{r}}^{\prime}\right) d \tilde{V}^{\prime}=\mathbf{0} \quad \forall \tilde{\mathbf{r}} \in \tilde{\Omega},
\end{aligned}
$$


where it was used that $\mathbf{v}_{h}^{(1)}=\mathbf{v}_{h}^{(1) \perp}$ [according to Eq. (E4)], the charge neutrality condition [Eq. (3) of the main paper], and the fact that the MQS modes have zero dipole moment [Eq. (11) of the main paper]. Since $\kappa_{h}^{\perp}$ is an eigenvalue of the left-hand side of Eq. (E12) (in the weak form in the space of transverse functions), the normal solvability condition states that a solution of Eq. (E12) exists only under the condition that the its right-hand side is orthogonal to the corresponding eigencurrent $\mathbf{j}_{h}^{\perp}$. Consequently, we have

$$
\kappa_{h}^{(1)} \frac{1}{4 \pi} \int_{\tilde{\Omega}} \mathbf{j}_{h}^{\perp}(\tilde{\mathbf{r}}) \cdot \int_{\tilde{\Omega}} \Delta \tilde{\mathbf{r}}^{-1} \mathbf{j}_{h}^{\perp}\left(\tilde{\mathbf{r}}^{\prime}\right) d \tilde{V}^{\prime} d \tilde{V}=\frac{\kappa_{h}^{(1)}}{\kappa_{h}^{\perp}}\left\|\mathbf{j}_{h}^{\perp}\right\|^{2}=0 .
$$

Thus, irrespective of the shape of the object, the first-order correction is always zero:

$$
\begin{gathered}
\kappa_{h}^{(1)}=0, \\
\mathbf{v}_{h}^{(1)}(\tilde{\mathbf{r}})=\mathbf{0}, \quad \forall \tilde{\mathbf{r}} \in \tilde{\Omega} .
\end{gathered}
$$

By using Eq. (E14) and testing Eq. (E12) in the space of longitudinal functions, it is obtained that

$$
v_{h n}^{(3)}=0 .
$$

\section{Fourth order}

Collecting the fourth-order terms in Eq. (38) and exploiting Eqs. (E2), (E6), (E7), and (E14)-(E16), the following integrodifferential equation for the second-order correction of the current density mode $\mathbf{v}_{h}^{(2)}$ is obtained:

$$
\begin{aligned}
& 4 \pi \mathbf{v}_{h}^{(2)}(\tilde{\mathbf{r}})-\kappa_{h}^{\perp} \int_{\tilde{\Omega}} \Delta \tilde{\mathbf{r}}^{-1} \mathbf{v}_{h}^{(2) \perp}\left(\tilde{\mathbf{r}}^{\prime}\right) d \tilde{V}^{\prime} \\
& \quad=-\kappa_{h}^{\perp} \tilde{\nabla} \oint_{\partial \tilde{\Omega}} \Delta \tilde{\mathbf{r}}^{-1} v_{h n}^{(4)}\left(\tilde{\mathbf{r}}^{\prime}\right) d \tilde{S}^{\prime}-\kappa_{h}^{(2)} \tilde{\nabla} \oint_{\partial \tilde{\Omega}} \Delta \tilde{\mathbf{r}}^{-1} v_{h n}^{(2)}\left(\tilde{\mathbf{r}}^{\prime}\right) d \tilde{S}^{\prime}+\kappa_{h}^{\perp} \tilde{\nabla} \oint_{\partial \tilde{\Omega}} \frac{\Delta \tilde{\mathbf{r}}}{2} v_{h n}^{(2)}\left(\tilde{\mathbf{r}}^{\prime}\right) d \tilde{S}^{\prime} \\
& \quad-\kappa_{h}^{\perp} \int_{\tilde{\Omega}} \frac{\Delta \tilde{\mathbf{r}}}{2} \mathbf{j}_{h}^{\perp}\left(\tilde{\mathbf{r}}^{\prime}\right) d \tilde{V}^{\prime}+\kappa_{h}^{\perp} \int_{\tilde{\Omega}} \Delta \tilde{\mathbf{r}}^{-1} \mathbf{v}_{h}^{(2) \|}\left(\tilde{\mathbf{r}}^{\prime}\right) d \tilde{V}^{\prime}+\kappa_{h}^{(2)} \int_{\tilde{\Omega}} \Delta \tilde{\mathbf{r}}^{-1} \mathbf{j}_{h}^{\perp}\left(\tilde{\mathbf{r}}^{\prime}\right) d \tilde{V}^{\prime} \quad \forall \tilde{\mathbf{r}} \in \tilde{\Omega},
\end{aligned}
$$

where the second-order correction $\mathbf{v}_{h}^{(2)}$ has been decomposed into its longitudinal and transverse components, i.e., $\mathbf{v}_{h}^{(2)}=\mathbf{v}_{h}^{(2) \|}+$ $\mathbf{v}_{h}^{(2) \perp}$. Since $\kappa_{h}^{\perp}$ is an eigenvalue of the left-hand side of Eq. (E17), the normal solvability condition states that a solution of Eq. (E17) in the weak form in the space of transverse functions exists only under the condition that the its right-hand side is orthogonal to the corresponding current mode $\mathbf{j}_{h}^{\perp}$. Consequently, by enforcing the orthogonality of the right-hand side with $\mathbf{j}_{h}^{\perp}$, and expanding $\mathbf{v}_{h}^{(2) \|}$ in terms of the EQS basis set $\left\{\mathbf{j}_{h}^{\|}\right\}_{k \in \mathbb{N}}$ according to Eq. (E8), the following equation is obtained:

$$
\begin{aligned}
& \kappa_{h}^{(2)} \int_{\tilde{\Omega}} \mathbf{j}_{h}^{\perp}(\tilde{\mathbf{r}}) \cdot \int_{\tilde{\Omega}} \Delta \tilde{\mathbf{r}}^{-1} \mathbf{j}_{h}^{\perp}\left(\tilde{\mathbf{r}}^{\prime}\right) d \tilde{V}^{\prime} d \tilde{V} \\
& \quad=\kappa_{h}^{\perp} \int_{\tilde{\Omega}} \mathbf{j}_{h}^{\perp}(\tilde{\mathbf{r}}) \cdot \int_{\tilde{\Omega}} \frac{\Delta \tilde{\mathbf{r}}}{2} \mathbf{j}_{h}^{\perp}\left(\tilde{\mathbf{r}}^{\prime}\right) d \tilde{V}^{\prime}-\kappa_{h}^{\perp} \sum_{k=1}^{\infty} \alpha_{k}^{(2)} \int_{\tilde{\Omega}} \mathbf{j}_{k}^{\|}\left(\tilde{\mathbf{r}}^{\prime}\right) \cdot \int_{\tilde{\Omega}} \frac{\mathbf{j}_{h}^{\perp}(\tilde{\mathbf{r}})}{\left|\tilde{\mathbf{r}}-\tilde{\mathbf{r}}^{\prime}\right|} d \tilde{V}^{\prime} d \tilde{V}
\end{aligned}
$$

Replacing the explicit expression of $\alpha_{h, k}^{(2)}$, by using Eq. (E9), the second-order correction is obtained:

$$
\kappa_{h}^{(2)}=\frac{\left(\kappa_{h}^{\perp}\right)^{2}}{4 \pi} \frac{1}{\left\|\mathbf{j}_{h}^{\perp}\right\|^{2}}\left(\int_{\tilde{\Omega}} \mathbf{j}_{h}^{\perp}(\tilde{\mathbf{r}}) \cdot \int_{\tilde{\Omega}} \frac{\left|\tilde{\mathbf{r}}-\tilde{\mathbf{r}}^{\prime}\right|}{2} \mathbf{j}_{h}^{\perp}\left(\tilde{\mathbf{r}}^{\prime}\right) d \tilde{V}^{\prime} d \tilde{V}+\sum_{k=1}^{\infty} \frac{\chi_{k}^{\|}}{4 \pi} \frac{1}{\left\|\mathbf{j}_{k}^{\|}\right\|^{2}}\left|\int_{\tilde{\Omega}} \mathbf{j}_{k}^{\|}(\tilde{\mathbf{r}}) \cdot \int_{\tilde{\Omega}} \frac{\mathbf{j}_{h}^{\perp}\left(\tilde{\mathbf{r}}^{\prime}\right)}{\left|\tilde{\mathbf{r}}-\tilde{\mathbf{r}}^{\prime}\right|} d \tilde{V}^{\prime} d \tilde{V}\right|^{2}\right) .
$$

The second-order radiative correction $\kappa_{h}^{(2)}$ originates from two contribution: The first term in parentheses in Eq. (E19) represents the radiative self-coupling of the volumetric current density $\mathbf{j}_{h}^{\perp}$ mediated by the vector potential; the second term is instead is associated to the magnetostatic interaction energy between the MQS current mode $\mathbf{j}_{h}^{\perp}$ and the EQS current mode $\mathbf{j}_{k}^{\|}$. For " $\mathbb{A}^{\perp}$ " modes, Eq. (E19) further simplifies:

$$
\kappa_{h}^{(2)}=\frac{\left(\kappa_{h}^{\perp}\right)^{2}}{4 \pi} \frac{1}{\left\|\mathbf{j}_{h}^{\perp}\right\|^{2}} \int_{\tilde{\Omega}} \mathbf{j}_{h}^{\perp}(\tilde{\mathbf{r}}) \cdot \int_{\tilde{\Omega}} \frac{\left|\tilde{\mathbf{r}}-\tilde{\mathbf{r}}^{\prime}\right|}{2} \mathbf{j}_{h}^{\perp}\left(\tilde{\mathbf{r}}^{\prime}\right) d \tilde{V}^{\prime} d \tilde{V} \quad \text { for } \mathbb{A}^{\perp} \text { modes. }
$$

Once the condition (E19) is satisfied, the normal solvability condition guarantees the existence of a solution of Eq. (E17). Its expression is now derived. The transverse part $\mathbf{v}_{h}^{(2) \perp}$ belongs to the functional space spanned by the MQS current modes $\left\{\mathbf{j}_{k}^{\perp}\right\}_{k \in \mathbb{N}}$, and therefore it may be expanded as

$$
\mathbf{v}_{h}^{(2) \perp}=\sum_{k=1}^{\infty} \beta_{h k}^{(2)} \mathbf{j}_{k}^{\perp}
$$


By substituting the expansion (E21) into Eq. (E17), and then solving the resulting equation in the weak form by testing it using the set of MQS modes $\left\{\mathbf{j}_{k}^{\perp}\right\}_{\in \mathbb{N}}$, it is obtained that $\beta_{h, h}^{(2)}=0$ and

$$
\beta_{h, k}^{(2)}=\frac{\kappa_{k}^{\perp} \kappa_{h}^{\perp}}{\kappa_{h}^{\perp}-\kappa_{k}^{\perp}} \frac{1}{4 \pi} \frac{1}{\left\|\mathbf{j}_{k}^{\perp}\right\|^{2}}\left[\int_{\tilde{\Omega}} \mathbf{j}_{k}^{\perp}(\tilde{\mathbf{r}}) \cdot \int_{\tilde{\Omega}} \frac{\left|\tilde{\mathbf{r}}-\tilde{\mathbf{r}}^{\prime}\right|}{2} \mathbf{j}_{h}^{\perp}\left(\tilde{\mathbf{r}}^{\prime}\right) d \tilde{V}^{\prime} d \tilde{V}-\sum_{s=1}^{\infty} \alpha_{h, s}^{(2)} \int_{\tilde{\Omega}} \mathbf{j}_{s}^{\|}\left(\tilde{\mathbf{r}}^{\prime}\right) \cdot \int_{\tilde{\Omega}} \frac{\mathbf{j}_{k}^{\perp}(\tilde{\mathbf{r}})}{\left|\tilde{\mathbf{r}}-\tilde{\mathbf{r}}^{\prime}\right|} d \tilde{V}^{\prime} d \tilde{V}\right] .
$$

Replacing the explicit expression of $\alpha_{h, k}^{(2)}$, found in Eq. (E9), Eq. (E22) becomes

$$
\begin{aligned}
\beta_{h, k}^{(2)}= & \frac{\kappa_{k}^{\perp} \kappa_{h}^{\perp}}{\kappa_{h}^{\perp}-\kappa_{k}^{\perp}} \frac{1}{4 \pi} \frac{1}{\left\|\mathbf{j}_{k}^{\perp}\right\|^{2}}\left[\int_{\tilde{\Omega}} \mathbf{j}_{k}^{\perp}(\tilde{\mathbf{r}}) \cdot \int_{\tilde{\Omega}} \frac{\left|\tilde{\mathbf{r}}-\tilde{\mathbf{r}}^{\prime}\right|}{2} \mathbf{j}_{h}^{\perp}\left(\tilde{\mathbf{r}}^{\prime}\right) d \tilde{V}^{\prime} d \tilde{V}\right. \\
& \left.+\sum_{s=1}^{\infty} \frac{\chi_{s}^{\|}}{4 \pi} \frac{1}{\left\|\mathbf{j}_{s}^{\|}\right\|^{2}}\left(\int_{\tilde{\Omega}} \mathbf{j}_{s}^{\|}\left(\tilde{\mathbf{r}}^{\prime}\right) \cdot \int_{\tilde{\Omega}} \frac{\mathbf{j}_{k}^{\perp}(\tilde{\mathbf{r}})}{\left|\tilde{\mathbf{r}}-\tilde{\mathbf{r}}^{\prime}\right|} d \tilde{V}^{\prime} d \tilde{V}\right)\left(\int_{\tilde{\Omega}} \mathbf{j}_{s}^{\|}\left(\tilde{\mathbf{r}}^{\prime}\right) \cdot \int_{\tilde{\Omega}} \frac{\mathbf{j}_{h}^{\perp}(\tilde{\mathbf{r}})}{\left|\tilde{\mathbf{r}}-\tilde{\mathbf{r}}^{\prime}\right|} d \tilde{V}^{\prime} d \tilde{V}\right)\right] .
\end{aligned}
$$

\section{Fifth order}

By matching the fifth-order terms in Eq. (38) and using Eqs. (E2), (E6), (E7), and (E14)-(E16), together with the charge neutrality condition of EQS modes and the property that the MQS modes have zero dipole moment, the following equation is found:

$$
\begin{aligned}
4 \pi \mathbf{v}_{h}^{(3)}(\tilde{\mathbf{r}})-\kappa_{h}^{\perp} \int_{\tilde{\Omega}} \Delta \tilde{\mathbf{r}}^{-1} \mathbf{v}_{h}^{(3) \perp}\left(\tilde{\mathbf{r}}^{\prime}\right) d \tilde{V}^{\prime}= & -\kappa_{h}^{\perp} \tilde{\nabla} \oint_{\partial \tilde{\Omega}} \Delta \tilde{\mathbf{r}}^{-1} v_{h n}^{(5)}\left(\tilde{\mathbf{r}}^{\prime}\right) d \tilde{S}^{\prime}-i \kappa_{h}^{\perp} \tilde{\nabla} \oint_{\partial \tilde{\Omega}} \frac{\Delta \tilde{\mathbf{r}}^{2}}{3 !} v_{h n}^{(2)}\left(\tilde{\mathbf{r}}^{\prime}\right) d \tilde{S}^{\prime}-i \kappa_{h}^{\perp} \int_{\tilde{\Omega}} \mathbf{v}_{h}^{(2) \|}\left(\tilde{\mathbf{r}}^{\prime}\right) d \tilde{V}^{\prime} \\
& +\kappa_{h}^{(3)} \int_{\tilde{\Omega}} \Delta \tilde{\mathbf{r}}^{-1} \mathbf{j}_{h}^{\perp}\left(\tilde{\mathbf{r}}^{\prime}\right) d \tilde{V}^{\prime}+i \kappa_{h}^{\perp} \int_{\tilde{\Omega}} \frac{\Delta \tilde{\mathbf{r}}^{2}}{3 !} \mathbf{j}_{h}^{\perp}\left(\tilde{\mathbf{r}}^{\prime}\right) d \tilde{V}^{\prime} \quad \forall \tilde{\mathbf{r}} \in \tilde{\Omega} .
\end{aligned}
$$

Since $\kappa_{h}^{\perp}$ is an eigenvalue of the left-hand side of (E24) (in the weak form in the space of transverse functions), the normal solvability condition states that a solution of Eq. (E24) exists only under the condition that the its right-hand side is orthogonal to the corresponding eigencurrent $\mathbf{j}_{h}^{\perp}$. Thus, by projecting the right-hand side of Eq. (E24) along $\mathbf{j}_{h}^{\perp}$, the following equation is obtained:

$$
-\kappa_{h}^{(3)} \int_{\tilde{\Omega}} \mathbf{j}_{h}^{\perp}(\tilde{\mathbf{r}}) \cdot \int_{\tilde{\Omega}} \Delta \tilde{\mathbf{r}}^{-1} \mathbf{j}_{h}^{\perp}\left(\tilde{\mathbf{r}}^{\prime}\right) d \tilde{V}^{\prime} d \tilde{V}-i \kappa_{h}^{\perp} \int_{\tilde{\Omega}} \mathbf{j}_{h}^{\perp}(\tilde{\mathbf{r}}) \cdot \int_{\tilde{\Omega}} \frac{\Delta \tilde{\mathbf{r}}^{2}}{3 !} \mathbf{j}_{h}^{\perp}\left(\tilde{\mathbf{r}}^{\prime}\right) d \tilde{V}^{\prime} d \tilde{V}=0 .
$$

By using the fact that $\mathbf{j}_{h}^{\perp}$ is a MQS current mode, the explicit expression for $\kappa_{h}^{(3)}$ is found:

$$
\kappa_{h}^{(3)}=-i \frac{\left(\kappa_{h}^{\perp}\right)^{2}}{\left\|\mathbf{j}_{h}^{\perp}\right\|^{2}} \frac{1}{4 \pi} \int_{\tilde{\Omega}} \mathbf{j}_{h}^{\perp}(\tilde{\mathbf{r}}) \cdot \int_{\tilde{\Omega}} \frac{\left|\tilde{\mathbf{r}}-\tilde{\mathbf{r}}^{\prime}\right|^{2}}{6} \mathbf{j}_{h}^{\perp}\left(\tilde{\mathbf{r}}^{\prime}\right) d \tilde{V}^{\prime} d \tilde{V} .
$$

The above expression can be further manipulated, and eventually rewritten in terms of the multipolar components of the quasistatic mode. By using the identity $\left|\tilde{\mathbf{r}}-\tilde{\mathbf{r}}^{\prime}\right|^{2}=|\tilde{\mathbf{r}}|^{2}+\left|\tilde{\mathbf{r}}^{\prime}\right|^{2}-2 \tilde{\mathbf{r}} \cdot \tilde{\mathbf{r}}^{\prime}$, and the fact that the current distribution $\mathbf{j}_{h}^{\perp}$ has vanishing electric dipole and electric quadrupole moments, the following identity is obtained:

$$
\int_{\tilde{\Omega}} \mathbf{j}_{h}^{\perp}(\tilde{\mathbf{r}}) \cdot \int_{\tilde{\Omega}} \frac{\left|\tilde{\mathbf{r}}-\tilde{\mathbf{r}}^{\prime}\right|^{2}}{6} \mathbf{j}_{h}^{\perp}\left(\tilde{\mathbf{r}}^{\prime}\right) d \tilde{V}^{\prime} d \tilde{V}=-\frac{1}{6}\left(\int_{\tilde{\Omega}} \mathbf{j}_{h}^{\perp}(\tilde{\mathbf{r}}) \times \tilde{\mathbf{r}} d \tilde{V}\right) \cdot\left(\int_{\tilde{\Omega}} \mathbf{j}_{h}^{\perp}\left(\tilde{\mathbf{r}}^{\prime}\right) \times \tilde{\mathbf{r}}^{\prime} d \tilde{V}^{\prime}\right)=-\frac{2}{3}\left\|\mathbf{P}_{\mathrm{M} \mid h}^{\perp}\right\|^{2} .
$$

By using the above identity, Eq. (E26) becomes

$$
\kappa_{h}^{(3)}=i \frac{\left(\kappa_{h}^{\perp}\right)^{2}}{\left\|\mathbf{j}_{h}^{\perp}\right\|^{2}} \frac{1}{6 \pi}\left\|\mathbf{P}_{\mathrm{M} \mid h}^{\perp}\right\|^{2}
$$

where $\mathbf{P}_{\mathrm{M} \mid h}^{\perp}$ is the magnetic dipole moment of the $h$ th MQS mode defined in Eq. (A3) in Appendix A.

The corresponding correction to the current density mode can be now derived. The transverse current density $\mathbf{v}_{h}^{(3) \perp}$ may be expanded in terms of the MQS current modes as

$$
\mathbf{v}_{h}^{(3) \perp}=\sum_{k=1}^{\infty} \beta_{h k}^{(3)} \mathbf{j}_{k}^{\perp} .
$$

By substituting Eq. (E29) in Eq. (E24) and testing the resulting equation in the space of transverse functions, spanned by the magnetoquasistatic modes $\left\{\mathbf{j}_{h}^{\perp}\right\}_{k \in \mathbb{N}}$, it is found that $\beta_{h, h}^{(3)}=0$ and

$$
\beta_{h, k}^{(3)}=-i \frac{\kappa_{k}^{\perp} \kappa_{h}^{\perp}}{\kappa_{h}^{\perp}-\kappa_{k}^{\perp}} \frac{1}{4 \pi} \frac{1}{\left\|\mathbf{j}_{k}^{\perp}\right\|^{2}} \int_{\tilde{\Omega}} \mathbf{j}_{k}^{\perp}(\tilde{\mathbf{r}}) \cdot \int_{\tilde{\Omega}} \frac{\left|\tilde{\mathbf{r}}-\tilde{\mathbf{r}}^{\prime}\right|^{2}}{3 !} \mathbf{j}_{h}^{\perp}\left(\tilde{\mathbf{r}}^{\prime}\right) d \tilde{V}^{\prime} d \tilde{V}, \quad \forall h \neq k .
$$


Equation (E30) can be rewritten in terms of the magnetic dipoles $\mathbf{P}_{\mathrm{M} \mid h}^{\perp}$ and $\mathbf{P}_{\mathrm{M} \mid k}^{\perp}$ of the $h$ th and $k$ th MQS modes, respectively:

$$
\beta_{h, k}^{(3)}=i \frac{1}{6 \pi} \frac{1}{\left\|\mathbf{j}_{k}^{\perp}\right\|^{2}} \frac{\kappa_{k}^{\perp} \kappa_{h}^{\perp}}{\kappa_{h}^{\perp}-\kappa_{k}^{\perp}} \mathbf{P}_{\mathrm{M} \mid h}^{\perp} \cdot \mathbf{P}_{\mathrm{M} \mid k}^{\perp} .
$$

Starting from Eq. (E24), it is also possible to derive the longitudinal component of the fifth-order mode correction, namely $\mathbf{v}_{h}^{(5)}$. Indeed, by testing Eq. (E24) in the space of longitudinal function, which is spanned by the EQS current modes, and expanding $\mathbf{v}_{h}^{(5)}$ in the same EQS basis, i.e.,

$$
\mathbf{v}_{h}^{(5) \|}=\sum_{k=1}^{\infty} \alpha_{h, k}^{(5)} \mathbf{j}_{k}^{\|},
$$

it is obtained that

$$
\begin{aligned}
- & \kappa_{h}^{\perp} \sum_{s=1}^{\infty} \alpha_{h, s}^{(5)} \int_{\tilde{\Omega}} \mathbf{j}_{k}^{\|}(\tilde{\mathbf{r}}) \cdot \tilde{\nabla} \oint_{\partial \tilde{\Omega}} \Delta \tilde{\mathbf{r}}^{-1} \mathbf{j}_{s}^{\|}\left(\tilde{\mathbf{r}}^{\prime}\right) d \tilde{S}^{\prime} d \tilde{V}=i \kappa_{h}^{\perp} \sum_{s=1}^{\infty} \alpha_{h, s}^{(2)} \int_{\tilde{\Omega}} \mathbf{j}_{k}^{\|}(\tilde{\mathbf{r}}) \cdot \tilde{\nabla} \oint_{\partial \tilde{\Omega}} \frac{\Delta \tilde{\mathbf{r}}^{2}}{3 !} \mathbf{j}_{s}^{\|}\left(\tilde{\mathbf{r}}^{\prime}\right) d \tilde{S}^{\prime} d \tilde{V} \\
& +i \kappa_{h}^{\perp} \int_{\tilde{\Omega}} \mathbf{j}_{k}^{\|}(\tilde{\mathbf{r}}) \cdot \int_{\tilde{\Omega}} \mathbf{v}_{h}^{(2) \|}\left(\tilde{\mathbf{r}}^{\prime}\right) d \tilde{V}^{\prime} d \tilde{V}-\kappa_{h}^{\perp} \sum_{s=1}^{\infty} \beta_{h, s}^{(3)} \int_{\tilde{\Omega}} \mathbf{j}_{k}^{\|}(\tilde{\mathbf{r}}) \cdot \int_{\tilde{\Omega}} \Delta \tilde{\mathbf{r}}^{-1} \mathbf{j}_{s}^{\perp}\left(\tilde{\mathbf{r}}^{\prime}\right) d \tilde{V}^{\prime} d \tilde{V} \\
& -\kappa_{h}^{(3)} \int_{\tilde{\Omega}} \mathbf{j}_{k}^{\|}(\tilde{\mathbf{r}}) \cdot \int_{\tilde{\Omega}} \Delta \tilde{\mathbf{r}}^{-1} \mathbf{j}_{h}^{\perp}\left(\tilde{\mathbf{r}}^{\prime}\right) d \tilde{V}^{\prime} d \tilde{V}-i \kappa_{h}^{\perp} \int_{\tilde{\Omega}} \mathbf{j}_{k}^{\|}(\tilde{\mathbf{r}}) \cdot \int_{\tilde{\Omega}} \frac{\Delta \tilde{\mathbf{r}}^{2}}{3 !} \mathbf{j}_{h}^{\perp}\left(\tilde{\mathbf{r}}^{\prime}\right) d \tilde{V}^{\prime} d \tilde{V}
\end{aligned}
$$

Assuming that the $h$ th MQS mode has vanishing magnetic dipole moment, namely $\mathbf{P}_{\mathrm{M} \mid h}^{\perp}=\mathbf{0}$, then $\kappa_{h}^{(3)}=0$ and $\beta_{h, k}^{(3)}=0$. Thus, Eq. (E33) becomes

$$
\begin{aligned}
\alpha_{h, k}^{(5)}= & i \frac{\chi_{k}^{\|}}{\left\|\mathbf{j}_{k}^{\|}\right\|^{2}} \frac{1}{4 \pi}\left[\sum_{s=1}^{\infty} \alpha_{h, s}^{(2)} \oint_{\partial \tilde{\Omega}} j_{n, k}^{\|}(\tilde{\mathbf{r}}) \oint_{\partial \tilde{\Omega}} \frac{\Delta \tilde{\mathbf{r}}^{2}}{3 !} j_{n, s}^{\|}\left(\tilde{\mathbf{r}}^{\prime}\right) d \tilde{S} d \tilde{S}^{\prime}+\sum_{s=1}^{\infty} \alpha_{h, s}^{(2)}\left(\int_{\tilde{\Omega}} \mathbf{j}_{k}^{\|}(\tilde{\mathbf{r}}) d \tilde{V}\right) \cdot\left(\int_{\tilde{\Omega}} \mathbf{j}_{s}^{\|}\left(\tilde{\mathbf{r}}^{\prime}\right) d \tilde{V}^{\prime}\right)\right. \\
& \left.-\int_{\tilde{\Omega}} \mathbf{j}_{k}^{\|}(\tilde{\mathbf{r}}) \cdot \int_{\tilde{\Omega}} \frac{\left|\tilde{\mathbf{r}}-\tilde{\mathbf{r}}^{\prime}\right|^{2}}{3 !} \mathbf{j}_{h}^{\perp}\left(\tilde{\mathbf{r}}^{\prime}\right) d \tilde{V}^{\prime} d \tilde{V}\right] .
\end{aligned}
$$

By assuming that $\mathbf{j}_{h}^{\perp}$ has zero magnetic dipole moment, and by exploiting the fact that $\mathbf{j}_{h}^{\perp}$ has zero electric dipole and electric quadrupole moments, while $\mathbf{j}_{k}^{\|}$has zero magnetic dipole moment, the following identities can be demonstrated:

$$
\begin{gathered}
\oint_{\partial \tilde{\Omega}} j_{n, k}^{\|}(\tilde{\mathbf{r}}) \oint_{\partial \tilde{\Omega}} \frac{\Delta \tilde{\mathbf{r}}^{2}}{3 !} j_{n, s}^{\|}\left(\tilde{\mathbf{r}}^{\prime}\right) d \tilde{S} d \tilde{S}^{\prime}=-\frac{1}{3} \mathbf{P}_{\mathrm{E} \mid k}^{\|} \cdot \mathbf{P}_{\mathrm{E} \mid s}^{\|}, \\
\left(\int_{\tilde{\Omega}} \mathbf{j}_{k}^{\|}(\tilde{\mathbf{r}}) d \tilde{V}\right) \cdot\left(\int_{\tilde{\Omega}} \mathbf{j}_{s}^{\|}\left(\tilde{\mathbf{r}}^{\prime}\right) d \tilde{V}^{\prime}\right)=\mathbf{P}_{\mathrm{E} \mid k}^{\|} \cdot \mathbf{P}_{\mathrm{E} \mid s}^{\|}, \\
\int_{\tilde{\Omega}} \mathbf{j}_{k}^{\|}(\tilde{\mathbf{r}}) \cdot \int_{\tilde{\Omega}} \frac{\left|\tilde{\mathbf{r}}-\tilde{\mathbf{r}}^{\prime}\right|^{2}}{3 !} \mathbf{j}_{h}^{\perp}\left(\tilde{\mathbf{r}}^{\prime}\right) d \tilde{V}^{\prime} d \tilde{V}=\frac{2}{3} \mathbf{P}_{\mathrm{E} 2 \mid h}^{\perp} \cdot \mathbf{P}_{\mathrm{E} \mid k}^{\|} .
\end{gathered}
$$

By using the above identities in Eq. (E34), it is obtained that

$$
\alpha_{h, k}^{(5)}=i \frac{1}{6 \pi} \frac{\chi_{k}^{\|}}{\left\|\mathbf{j}_{k}^{\|}\right\|^{2}}\left[\left(\sum_{s=1}^{\infty} \alpha_{h, s}^{(2)} \mathbf{P}_{\mathrm{E} \mid s}^{\|}\right)-\mathbf{P}_{\mathrm{E} 2 \mid h}^{\perp}\right] \cdot \mathbf{P}_{\mathrm{E} \mid k}^{\|} .
$$

By recalling that the dipole moment of the second-order mode correction is [see Eq. (E11)]

$$
\mathbf{P}_{\mathrm{E} \mid h}^{(2)}=\sum_{s=1}^{\infty} \alpha_{h, s}^{(2)} \mathbf{P}_{\mathrm{E} \mid s}^{\|}
$$

Equation (E38) has the final form

$$
\alpha_{h, k}^{(5)}=i \frac{1}{6 \pi} \frac{\chi_{k}^{\|}}{\left\|\mathbf{j}_{k}^{\|}\right\|^{2}}\left[\mathbf{P}_{\mathrm{E} \mid h}^{(2)}-\mathbf{P}_{\mathrm{E} 2 \mid h}^{\perp}\right] \cdot \mathbf{P}_{\mathrm{E} \mid k}^{\|} .
$$

\section{Seventh order}

As shown in the main paper, the MQS modes with zero magnetic dipole moment exhibit vanishing third order correction $\kappa_{h}^{(3)}$. In this case, to retrieve information about the radiative Q-factor, it is mandatory to consider the fifth-order perturbation $\kappa_{h}^{(5)}$. 
Collecting the seventh-order terms in Eq. (38), we arrive at the following integrodifferential equation for $\mathbf{v}_{h}^{(5)}$ :

$$
\begin{aligned}
4 \pi \mathbf{v}_{h}^{(5)} & -\kappa_{h}^{\perp} \int_{\tilde{\Omega}} \Delta \tilde{\mathbf{r}}^{-1} \mathbf{v}_{h}^{(5) \perp}\left(\tilde{\mathbf{r}}^{\prime}\right) d \tilde{V}^{\prime} \\
= & -\kappa_{h}^{(5)} \tilde{\nabla} \oint_{\partial \tilde{\Omega}} \Delta \tilde{\mathbf{r}}^{-1} v_{h n}^{(2)}\left(\tilde{\mathbf{r}}^{\prime}\right) d \tilde{S}^{\prime}+\kappa_{h}^{(3)} \tilde{\nabla} \oint_{\partial \tilde{\Omega}} \frac{\Delta \tilde{\mathbf{r}}}{2 !} v_{h n}^{(2)}\left(\tilde{\mathbf{r}}^{\prime}\right) d \tilde{S}^{\prime}-i \kappa_{h}^{(2)} \tilde{\nabla} \oint_{\partial \tilde{\Omega}} \frac{\Delta \tilde{\mathbf{r}}^{2}}{3 !} v_{h n}^{(2)}\left(\tilde{\mathbf{r}}^{\prime}\right) d \tilde{S}^{\prime} \\
& +i \kappa_{h}^{\perp} \tilde{\nabla} \oint_{\partial \tilde{\Omega}} \frac{\Delta \tilde{\mathbf{r}}^{4}}{5 !} v_{h n}^{(2)}\left(\tilde{\mathbf{r}}^{\prime}\right) d \tilde{S}^{\prime}-\kappa_{h}^{(3)} \tilde{\nabla} \oint_{\partial \tilde{\Omega}} \Delta \tilde{\mathbf{r}}^{-1} v_{h n}^{(4)}\left(\tilde{\mathbf{r}}^{\prime}\right) d \tilde{S}^{\prime}-i \kappa_{h}^{\perp} \tilde{\nabla} \oint_{\partial \tilde{\Omega}} \frac{\Delta \tilde{\mathbf{r}}^{2}}{3 !} v_{h n}^{(4)}\left(\tilde{\mathbf{r}}^{\prime}\right) d \tilde{S}^{\prime} \\
& +\kappa_{h}^{\perp} \tilde{\nabla} \oint_{\partial \tilde{\Omega}} \frac{\Delta \tilde{\mathbf{r}}}{2 !} v_{h n}^{(5)}\left(\tilde{\mathbf{r}}^{\prime}\right) d \tilde{S}^{\prime}-\kappa_{h}^{(2)} \tilde{\nabla} \oint_{\partial \tilde{\Omega}} \Delta \tilde{\mathbf{r}}^{-1} v_{h n}^{(5)}\left(\tilde{\mathbf{r}}^{\prime}\right) d \tilde{S}^{\prime}-\kappa_{h}^{\perp} \tilde{\nabla} \oint_{\partial \tilde{\Omega}} \Delta \tilde{\mathbf{r}}^{-1} v_{h n}^{(7)}\left(\tilde{\mathbf{r}}^{\prime}\right) d \tilde{S}^{\prime} \\
& +\kappa_{h}^{(5)} \int_{\tilde{\Omega}} \Delta \tilde{\mathbf{r}}^{-1} \mathbf{j}_{h}^{\perp}\left(\tilde{\mathbf{r}}^{\prime}\right) d \tilde{V}^{\prime}-i \kappa_{h}^{\perp} \int_{\tilde{\Omega}} \frac{\Delta \tilde{\mathbf{r}}^{4}}{5 !} \mathbf{j}_{h}^{\perp} d \tilde{V}^{\prime}+\kappa_{h}^{(3)} \int_{\tilde{\Omega}} \Delta \tilde{\mathbf{r}}^{-1} \mathbf{v}_{h}^{(2)} d \tilde{V}^{\prime} \\
& -i \kappa_{h}^{(2)} \int_{\tilde{\Omega}} \mathbf{v}_{h}^{(2)}\left(\tilde{\mathbf{r}}^{\prime}\right) d \tilde{V}^{\prime}+i \kappa_{h}^{\perp} \int_{\tilde{\Omega}} \frac{\Delta \tilde{\mathbf{r}}^{2}}{3 !} \mathbf{v}_{h}^{(2)}\left(\tilde{\mathbf{r}}^{\prime}\right) d \tilde{V}-\kappa_{h}^{\perp} \int_{\tilde{\Omega}} \frac{\Delta \tilde{\mathbf{r}}^{2}}{2 !} \mathbf{v}_{h}^{(3)} d \tilde{V}^{\prime} \\
& +\kappa_{h}^{(2)} \int_{\tilde{\Omega}} \Delta \tilde{\mathbf{r}}^{-1} \mathbf{v}_{h}^{(3)} d \tilde{V}^{\prime}-i \kappa_{h}^{\perp} \int_{\tilde{\Omega}} \mathbf{v}_{h}^{(4)}\left(\tilde{\mathbf{r}}^{\prime}\right) d \tilde{V}^{\prime}+\kappa_{h}^{\perp} \int_{\tilde{\Omega}} \Delta \tilde{\mathbf{r}}^{-1} \mathbf{v}_{h}^{(5) \|}\left(\tilde{\mathbf{r}}^{\prime}\right) d \tilde{V}^{\prime} .
\end{aligned}
$$

Since $\kappa_{h}^{\perp}$ is an eigenvalue of the left-hand side of Eq. (E41) (in the weak form in the space of transverse functions), the normal solvability condition states that a solution of Eq. (E41) exists only under the condition that the its right-hand side is orthogonal to the corresponding MQS mode $\mathbf{j}_{h}^{\perp}$. Thus, by projecting the right-hand side of Eq. (E41) along $\mathbf{j}_{h}^{\perp}$, the fifth-order correction is obtained:

$$
\begin{aligned}
\kappa_{h}^{(5)}= & \frac{\kappa_{h}^{\perp}}{\left\|\mathbf{j}_{h}^{\perp}\right\|^{2}} \frac{1}{4 \pi}\left(i \kappa_{h}^{\perp} \int_{\tilde{\Omega}} \mathbf{j}_{h}^{\perp}(\tilde{\mathbf{r}}) \cdot \int_{\tilde{\Omega}} \frac{\Delta \tilde{\mathbf{r}}^{4}}{5 !} \mathbf{j}_{h}^{\perp}\left(\tilde{\mathbf{r}}^{\prime}\right) d \tilde{V}^{\prime} d \tilde{V}-\kappa_{h}^{(3)} \int_{\tilde{\Omega}} \mathbf{j}_{h}^{\perp}(\tilde{\mathbf{r}}) \cdot \int_{\tilde{\Omega}} \Delta \tilde{\mathbf{r}}^{-1} \mathbf{v}_{h}^{(2)}\left(\tilde{\mathbf{r}}^{\prime}\right) d \tilde{V}^{\prime} d \tilde{V}+\right. \\
& -i \kappa_{h}^{\perp} \int_{\tilde{\Omega}} \mathbf{j}_{h}^{\perp}(\tilde{\mathbf{r}}) \cdot \int_{\tilde{\Omega}} \frac{\Delta \tilde{\mathbf{r}}^{2}}{3 !} \mathbf{v}_{h}^{(2)}\left(\tilde{\mathbf{r}}^{\prime}\right) d \tilde{V}^{\prime} d \tilde{V}+\kappa_{h}^{\perp} \int_{\tilde{\Omega}} \mathbf{j}_{h}^{\perp}(\tilde{\mathbf{r}}) \cdot \int_{\tilde{\Omega}} \frac{\Delta \tilde{\mathbf{r}}}{2 !} \mathbf{v}_{h}^{(3)}\left(\tilde{\mathbf{r}}^{\prime}\right) d \tilde{V}^{\prime} d \tilde{V} \\
& \left.-\kappa_{h}^{(2)} \int_{\tilde{\Omega}} \mathbf{j}_{h}^{\perp}(\tilde{\mathbf{r}}) \cdot \int_{\tilde{\Omega}} \Delta \tilde{\mathbf{r}}^{-1} \mathbf{v}_{h}^{(3)}\left(\tilde{\mathbf{r}}^{\prime}\right) d \tilde{V}^{\prime} d \tilde{V}-\kappa_{h}^{\perp} \int_{\tilde{\Omega}} \mathbf{j}_{h}^{\perp}(\tilde{\mathbf{r}}) \cdot \int_{\tilde{\Omega}} \Delta \tilde{\mathbf{r}}^{-1} \mathbf{v}_{h}^{(5) \|}\left(\tilde{\mathbf{r}}^{\prime}\right) d \tilde{V}^{\prime} d \tilde{V}\right) .
\end{aligned}
$$

If the $h$ th current mode has a vanishing magnetic dipole moment $\mathbf{P}_{\mathrm{M} \mid h}^{\perp}=\mathbf{0}$, then $\kappa_{h}^{(3)}=0$ and $\mathbf{v}_{h}^{(3)}=0$. Thus, Eq. (E42) becomes

$$
\begin{aligned}
\kappa_{h}^{(5)}= & i \frac{\left(\kappa_{h}^{\perp}\right)^{2}}{\left\|\mathbf{j}_{h}^{\perp}\right\|^{2}} \frac{1}{4 \pi}\left[\int_{\tilde{\Omega}} \mathbf{j}_{h}^{\perp}(\tilde{\mathbf{r}}) \cdot \int_{\tilde{\Omega}} \frac{\left|\tilde{\mathbf{r}}-\tilde{\mathbf{r}}^{\prime}\right|^{4}}{5 !} \mathbf{j}_{h}^{\perp}\left(\tilde{\mathbf{r}}^{\prime}\right) d \tilde{V}^{\prime} d \tilde{V}\right. \\
& \left.-\sum_{k=1}^{\infty} \alpha_{h, k}^{(2)} \int_{\tilde{\Omega}} \mathbf{j}_{k}^{\|}(\tilde{\mathbf{r}}) \cdot \int_{\tilde{\Omega}} \frac{\left|\tilde{\mathbf{r}}-\tilde{\mathbf{r}}^{\prime}\right|^{2}}{3 !} \mathbf{j}_{h}^{\perp}\left(\tilde{\mathbf{r}}^{\prime}\right) d \tilde{V}^{\prime} d \tilde{V}+i \sum_{k=1}^{\infty} \alpha_{h, k}^{(5)} \int_{\tilde{\Omega}} \mathbf{j}_{k}^{\|}(\tilde{\mathbf{r}}) \cdot \int_{\tilde{\Omega}} \frac{\mathbf{j}_{h}^{\perp}\left(\tilde{\mathbf{r}}^{\prime}\right)}{\left|\tilde{\mathbf{r}}-\tilde{\mathbf{r}}^{\prime}\right|} d \tilde{V}^{\prime} d \tilde{V} .\right]
\end{aligned}
$$

Equation (E43) can be rewritten in terms of the multipolar components of the MQS current mode $\mathbf{j}_{h}^{\perp}$. In order to achieve this goal, it is convenient to introduce some identities. Using Eq. (E9), it is obtained that

$$
\int_{\tilde{\Omega}} \mathbf{j}_{k}^{\|}(\tilde{\mathbf{r}}) \cdot \int_{\tilde{\Omega}} \frac{\mathbf{j}_{h}^{\perp}\left(\tilde{\mathbf{r}}^{\prime}\right)}{\left|\tilde{\mathbf{r}}-\tilde{\mathbf{r}}^{\prime}\right|} d \tilde{V} d \tilde{V}^{\prime}=-\frac{\alpha_{h, k}^{(2)}}{\chi_{k}^{\|}} 4 \pi\left\|\mathbf{j}_{k}^{\|}\right\|^{2} .
$$

Moreover, by using the following identities,

$$
\begin{gathered}
\left.\left.\left|\int_{\tilde{\Omega}}\right| \tilde{\mathbf{r}}\right|^{2} \mathbf{j}_{h}^{\perp}(\tilde{\mathbf{r}}) d \tilde{V}\right|^{2}=16\left\|\mathbf{P}_{\mathrm{E} 2 \mid h}^{\perp}\right\|^{2} \\
\int_{\tilde{\Omega}} \int_{\tilde{\Omega}}\left|\tilde{\mathbf{r}} \cdot \tilde{\mathbf{r}}^{\prime}\right|^{2} \mathbf{j}_{h}^{\perp}(\tilde{\mathbf{r}}) \cdot \mathbf{j}_{h}^{\perp}\left(\tilde{\mathbf{r}}^{\prime}\right) d \tilde{V} d \tilde{V}^{\prime}=\frac{3}{2} \sum_{i j}\left(Q_{\mathrm{M}|h| i j}^{\perp}\right)^{2}+12\left\|\mathbf{P}_{\mathrm{E} 2 \mid h}^{\perp}\right\|^{2}
\end{gathered}
$$

it is possible to prove that

$$
\int_{\tilde{\Omega}} \mathbf{j}_{h}^{\perp}(\tilde{\mathbf{r}}) \cdot \int_{\tilde{\Omega}} \frac{\left|\tilde{\mathbf{r}}-\tilde{\mathbf{r}}^{\prime}\right|^{4}}{5 !} \mathbf{j}_{h}^{\perp}\left(\tilde{\mathbf{r}}^{\prime}\right) d \tilde{V} d \tilde{V}^{\prime}=\frac{1}{20} \sum_{i j}\left(Q_{\mathrm{M}|h| i j}^{\perp}\right)^{2}+\frac{2}{3}\left\|\mathbf{P}_{\mathrm{E} 2 \mid h}^{\perp}\right\|^{2},
$$


where $Q_{\mathrm{M}|h| i j}^{\perp}$ are the components of the magnetic quadrupole $\overleftrightarrow{\mathbf{Q}}_{\mathrm{M} \mid h}^{\perp}$ of the $h$ th MQS mode, while $\mathbf{P}_{\mathrm{E} 2 \mid h}^{\perp}$ is the toroidal dipole of the $h$ th MQS mode. These multipoles are defined in Eqs. (A5) and (A4) in Appendix A, respectively. By using the identities (E44), (E47), and (E37), together with the expression of $\alpha_{h k}^{(5)}$, provided in Eq. (E38), Eq. (E43) becomes

$$
\begin{aligned}
\kappa_{h}^{(5)}= & i \frac{\left(\kappa_{h}^{\perp}\right)^{2}}{\left\|\mathbf{j}_{h}^{\perp}\right\|^{2}}\left[\frac{1}{80 \pi} \sum_{i j}\left(Q_{\mathrm{M}|h| i j}^{\perp}\right)^{2}+\frac{1}{6 \pi}\left\|\mathbf{P}_{\mathrm{E} 2 \mid h}^{\perp}\right\|^{2}-\frac{1}{6 \pi}\left(\sum_{k=1}^{\infty} \alpha_{h, k}^{(2)} \mathbf{P}_{\mathrm{E} \mid h}^{\|}\right) \cdot \mathbf{P}_{\mathrm{E} 2 \mid h}^{\perp}\right. \\
& \left.+\frac{1}{6 \pi}\left[\left(\sum_{s=1}^{\infty} \alpha_{h, s}^{(2)} \mathbf{P}_{\mathrm{E} \mid h}^{\|}\right)-\mathbf{P}_{\mathrm{E} 2 \mid h}^{\perp}\right] \cdot\left(\sum_{k=1}^{\infty} \alpha_{h, k}^{(2)} \mathbf{P}_{\mathrm{E} \mid h}^{\|}\right)\right] .
\end{aligned}
$$

Eventually, recalling the definition of the electric dipole moment $\mathbf{P}_{\mathrm{E} \mid h}^{(2)}$ of the second-order mode correction $\mathbf{v}_{h}^{(2)}$, introduced in Eq. (E11),

$$
\kappa_{h}^{(5)}=i \frac{\left(\kappa_{h}^{\perp}\right)^{2}}{\left\|\mathbf{j}_{h}^{\perp}\right\|^{2}}\left[\frac{1}{80 \pi} \sum_{i j}\left(Q_{\mathrm{M}|h| i j}^{\perp}\right)^{2}+\frac{1}{6 \pi}\left\|\mathbf{P}_{\mathrm{E} 2 \mid h}^{\perp}-\mathbf{P}_{\mathrm{E} \mid h}^{(2)}\right\|^{2}\right] .
$$

For $\mathbb{A}^{\perp}$ modes, the fifth-order correction simplifies as follows:

$$
\kappa_{h}^{(5)}=i \frac{\left(\kappa_{h}^{\perp}\right)^{2}}{\left\|\mathbf{j}_{h}^{\perp}\right\|^{2}}\left[\frac{1}{80 \pi} \sum_{i j}\left(Q_{\mathrm{M}|h| i j}^{\perp}\right)^{2}+\frac{1}{6 \pi}\left\|\mathbf{P}_{\mathrm{E} 2 \mid h}^{\perp}\right\|^{2}\right] \quad \text { for } \mathbb{A}^{\perp} \text { modes. }
$$

\section{APPENDIX F: SOME EXAMPLES OF CALCULATION OF RADIATION CORRECTIONS}

In this Appendix, the use of the radiation corrections formulas is exemplified for the electric and magnetic dipole modes of a sphere.

\section{Electric dipole mode of a sphere}

In this section, the radiation corrections are calculated for the electroquasistatic mode $\mathbf{j}_{e 01}^{\|}$of a sphere, which is associated to the EQS eigenvalue $\chi_{1}^{\|}=-3$. This mode is an electric dipole oriented along $\hat{\mathbf{z}}$. The analytic expression of the mode can be found by replacing $m=0$ and $n=1$ in Eq. (B2) of Appendix B, and it is reported below:

$$
\mathbf{j}_{e 01}^{\|}=\sqrt{\frac{3}{4 \pi}}(\cos \theta \hat{\mathbf{r}}-\sin \theta \hat{\boldsymbol{\theta}})=\sqrt{\frac{3}{4 \pi}} \hat{\mathbf{z}} .
$$

The corresponding surface charge density $j_{n, e 01}^{\|}=\mathbf{j}_{e 01}^{\|} \cdot \hat{\mathbf{n}}$ has the expression

$$
j_{n, e 01}^{\|}=\mathbf{j}_{e 01}^{\|} \cdot \hat{\mathbf{n}}=\sqrt{\frac{3}{4 \pi}} \cos \theta .
$$

The second-order correction $\chi_{1}^{(2)}$ is obtained by Eq. (29) of the main paper:

$$
\chi_{1}^{(2)}=-\left(\chi_{1}^{\|}\right)^{2} \frac{1}{4 \pi}\left(\oint_{\partial \tilde{\Omega}} j_{n, e 01}^{\|}(\tilde{\mathbf{r}}) \oint_{\partial \tilde{\Omega}} \frac{\left|\tilde{\mathbf{r}}-\tilde{\mathbf{r}}^{\prime}\right|}{2} j_{n, e 01}^{\|}\left(\tilde{\mathbf{r}}^{\prime}\right) d \tilde{S}^{\prime} d \tilde{S}+\int_{\tilde{\Omega}} \mathbf{j}_{e 01}^{\|}(\tilde{\mathbf{r}}) \cdot \int_{\tilde{\Omega}} \frac{\mathbf{j}_{e 01}^{\|}\left(\tilde{\mathbf{r}}^{\prime}\right)}{\left|\tilde{\mathbf{r}}-\tilde{\mathbf{r}}^{\prime}\right|} d \tilde{V}^{\prime} d \tilde{V}\right),
$$

Then, the first integral in parentheses is evaluated:

$$
\oint_{\partial \tilde{\Omega}} j_{n, e 01}^{\|}(\tilde{\mathbf{r}}) \oint_{\partial \tilde{\Omega}} \frac{\left|\tilde{\mathbf{r}}-\tilde{\mathbf{r}}^{\prime}\right|}{2} j_{n, e 01}^{\|}\left(\tilde{\mathbf{r}}^{\prime}\right) d \tilde{S} d \tilde{S}^{\prime}=-\frac{8}{15} \pi .
$$

Next, the second integral is computed:

$$
\int_{\tilde{\Omega}} \mathbf{j}_{e 01}^{\|}(\tilde{\mathbf{r}}) \cdot \int_{\tilde{\Omega}} \frac{\mathbf{j}_{e 01}^{\|}\left(\tilde{\mathbf{r}}^{\prime}\right)}{\left|\tilde{\mathbf{r}}-\tilde{\mathbf{r}}^{\prime}\right|} d \tilde{V}^{\prime} d \tilde{V}=\frac{3}{4 \pi} \int_{\tilde{\Omega}} \int_{\tilde{\Omega}} \frac{1}{\left|\tilde{\mathbf{r}}-\tilde{\mathbf{r}}^{\prime}\right|} d \tilde{V}^{\prime} d \tilde{V}
$$

In Eq. (F5), the inner volume integral is proportional to the electrostatic potential in the interior of a uniformly charged sphere, and thus it has the expression:

$$
\int_{\tilde{\Omega}} \frac{1}{\left|\tilde{\mathbf{r}}-\tilde{\mathbf{r}}^{\prime}\right|} d \tilde{V}^{\prime}=2 \pi\left(1-\frac{\tilde{r}^{2}}{3}\right),
$$

and thus by using Eq. (F6) in Eq. (F5) it is obtained that

$$
\int_{\tilde{\Omega}} \mathbf{j}_{e 01}^{\|}(\tilde{\mathbf{r}}) \cdot \int_{\tilde{\Omega}} \frac{\mathbf{j}_{e 01}^{\|}\left(\tilde{\mathbf{r}}^{\prime}\right)}{\left|\tilde{\mathbf{r}}-\tilde{\mathbf{r}}^{\prime}\right|} d \tilde{V}^{\prime} d \tilde{V}=\frac{3}{4 \pi} 4 \pi \int_{0}^{1} 2 \pi\left(1-\frac{\tilde{r}^{2}}{3}\right) \tilde{r}^{2} d \tilde{r}=6 \pi\left[\frac{\tilde{r}^{3}}{3}-\frac{\tilde{r}^{5}}{15}\right]_{0}^{1}=\frac{8}{5} \pi .
$$


Eventually, by using Eqs. (F4) and (F7) in Eq. (F3), the second-order correction is obtained:

$$
\chi_{1}^{(2)}=-\frac{9}{4 \pi}\left(\frac{8}{5} \pi-\frac{8}{15} \pi\right)=-\frac{9}{4 \pi}\left(\frac{16}{15} \pi\right)=-\frac{12}{5} .
$$

The third-order correction $\chi_{1}^{(3)}$ is given by Eq. (33) of the main paper:

$$
\chi_{1}^{(3)}=i \frac{1}{6 \pi}\left(\chi_{1}^{\|}\right)^{2}\left\|\mathbf{P}_{\mathrm{E}, 1}^{\|}\right\|^{2} .
$$

The electric dipole moment of the current distribution, following the definition (A1), is

$$
\mathbf{P}_{\mathrm{E} \mid 1}^{\|}=\int_{\tilde{\Omega}} \mathbf{j}_{e 01}^{\|} d \tilde{V}=\sqrt{\frac{4 \pi}{3}} \hat{\mathbf{z}} .
$$

Eventually, by using Eq. (F10) in Eq. (F9),

$$
\chi_{1}^{(3)}=i \frac{1}{6 \pi}\left(\chi_{1}^{\|}\right)^{2}\left\|\mathbf{P}_{\mathrm{E}, 1}^{\|}\right\|^{2}=i \frac{1}{6 \pi} 9\left(\frac{4 \pi}{3}\right)=2 i .
$$

\section{Magnetic dipole mode of a sphere}

Now, the radiation corrections are calculated for the magnetoquasistatic TE mode $\mathbf{j}_{e 011}^{\mathrm{TE} \perp}$ of a sphere, which is associated to the eigenvalue $\kappa_{11}^{\perp \mathrm{TE}}=\pi^{2}$. The mode analytic expression is given by Eq. (B6) in Appendix B, namely

$$
\begin{aligned}
\mathbf{j}_{e 011}^{\perp \mathrm{TE}}(\tilde{r}, \theta, \phi) & =\frac{1}{\sqrt{\beta_{011}}}\left[-\frac{d P_{1}^{0}(\cos \theta)}{d \theta}\right] j_{1}(\pi \tilde{r}) \hat{\boldsymbol{\phi}}, \\
\beta_{011} & =\frac{4}{3 \pi},
\end{aligned}
$$

where the value of $\beta_{011}$ guarantees the normalization $\left\|\mathbf{j}_{e 011}^{\perp \mathrm{TE}}\right\|=1$. By substituting in Eq. (F12) the expressions of the spherical Bessel function and of the associated Legendre functions, namely

$$
\begin{gathered}
j_{1}(z)=\left[\frac{\sin (z)}{z^{2}}-\frac{\cos z}{z}\right], \\
P_{1}^{0}(\cos \theta)=\cos \theta,
\end{gathered}
$$

the mode assumes the explicit form below:

$$
\mathbf{j}_{e 011}^{\perp \mathrm{TE}}(\tilde{r}, \theta, \phi)=\sqrt{\frac{3 \pi}{4}} \sin \theta\left[\frac{\sin (\pi \tilde{r})}{(\pi \tilde{r})^{2}}-\frac{\cos \pi \tilde{r}}{\pi \tilde{r}}\right] \hat{\boldsymbol{\phi}}
$$

The vector potential generated by the current mode $\mathbf{j}_{e 011}^{\perp \mathrm{TE}}$ does not have a component orthogonal to the particle surface, and thus it is an $\mathbb{A}^{\perp}$ mode. Thus, the second-order correction can be calculated by Eq. (43) of the main paper:

$$
\begin{aligned}
\kappa_{11}^{\mathrm{TE}(2)}= & \frac{\left(\kappa_{11}^{\perp \mathrm{TE}}\right)^{2}}{4 \pi}\left[\int_{\tilde{\Omega}} \mathbf{j}_{e 011}^{\perp \mathrm{TE}}(\tilde{\mathbf{r}})\right. \\
& \left.\cdot \int_{\tilde{\Omega}} \frac{\left|\tilde{\mathbf{r}}-\tilde{\mathbf{r}}^{\prime}\right|}{2} \mathbf{j}_{e 011}^{\perp \mathrm{TE}}\left(\tilde{\mathbf{r}}^{\prime}\right) d \tilde{V}^{\prime} d \tilde{V}\right]=-3 .
\end{aligned}
$$

The third-order correction $\kappa_{11}^{\mathrm{TE}(3)}$ can be calculated by Eq. (48) of the main paper:

$$
\kappa_{11}^{\mathrm{TE}(3)}=i\left(\kappa_{11}^{\perp \mathrm{TE}}\right)^{2} \frac{1}{6 \pi}\left\|\mathbf{P}_{\mathrm{M} \mid e 011}^{\perp}\right\|^{2} .
$$

In order to calculate the third-order correction, the magnetic dipole moment $\mathbf{P}_{\mathrm{M} \mid e 011}^{\perp \mathrm{TE}}$ of the current mode is indeed required. Using the definition (A3) in Appendix A, and considering that $\tilde{\boldsymbol{r}} \times \hat{\boldsymbol{\phi}}=-\tilde{r} \hat{\boldsymbol{\theta}}$,

$$
\begin{aligned}
\mathbf{P}_{\mathrm{M} \mid e 011}^{\perp \mathrm{TE}} & =\frac{1}{2} \int_{\tilde{\Omega}} \tilde{\mathbf{r}} \times \mathbf{j}_{e 011}^{\perp \mathrm{TE}}(\tilde{\mathbf{r}}) d \tilde{V} \\
& =-\sqrt{\frac{3 \pi}{4}} \frac{1}{2} \int_{\tilde{\Omega}} \sin \theta\left[\frac{\sin (\pi \tilde{r})}{(\pi \tilde{r})^{2}}-\frac{\cos \pi \tilde{r}}{\pi \tilde{r}}\right] \tilde{r} \hat{\boldsymbol{\theta}} d \tilde{V} .
\end{aligned}
$$

Using the fact $\hat{\boldsymbol{\theta}}=\cos \theta \cos \phi \hat{\mathbf{x}}+\cos \theta \sin \phi \hat{\mathbf{y}}-\sin \theta \hat{\mathbf{z}}$, and noting that the magnetic dipole moment components along $\hat{\mathbf{x}}$ and $\hat{\mathbf{y}}$ vanish due to the symmetry, Eq. (F18) becomes

$$
\begin{aligned}
\mathbf{P}_{\mathrm{M} \mid e 011}^{\perp \mathrm{TE}}= & \hat{\mathbf{z}} \sqrt{\frac{3 \pi}{4}} \frac{1}{2} \int_{\tilde{\Omega}} \tilde{r} \sin ^{2} \theta\left[\frac{\sin (\pi \tilde{r})}{(\pi \tilde{r})^{2}}-\frac{\cos \pi \tilde{r}}{\pi \tilde{r}}\right] d \tilde{V} \\
= & \hat{\mathbf{z}} \sqrt{\frac{3 \pi}{4}} \frac{1}{2} 2 \pi\left(\int_{0}^{1} \tilde{r}^{3}\left[\frac{\sin (\pi \tilde{r})}{(\pi \tilde{r})^{2}}-\frac{\cos \pi \tilde{r}}{\pi \tilde{r}}\right] d \tilde{r}\right) \\
& \times\left(\int_{0}^{\pi} \sin ^{3} \theta d \theta\right) \\
= & \hat{\mathbf{z}} \sqrt{\frac{3 \pi}{4}} \frac{1}{2} 2 \pi \frac{3}{\pi^{3}} \frac{4}{3}=\hat{\mathbf{z}} \sqrt{3 \pi} \frac{2}{\pi^{2}} .
\end{aligned}
$$

In conclusion, by combining (F17) and (F19):

$$
\kappa_{11}^{\mathrm{TE}(3)}=i\left(\kappa_{11}^{\perp \mathrm{TE}}\right)^{2} \frac{1}{6 \pi}\left\|\mathbf{P}_{\mathrm{M} \mid e 011}^{\perp \mathrm{TE}}\right\|^{2}=i \pi^{4} \frac{1}{6 \pi}\left(3 \pi \frac{4}{\pi^{4}}\right)=2 i .
$$

[1] D. R. Fredkin and I. D. Mayergoyz, Resonant Behavior of Dielectric Objects (Electrostatic Resonances), Phys. Rev. Lett. 91, 253902 (2003).

[2] D. J. Bergman and M. I. Stockman, Surface Plasmon Amplification by Stimulated Emission of Radiation: Quantum Generation of Coherent Surface Plasmons in Nanosystems, Phys. Rev. Lett. 90, 027402 (2003).

[3] K. Li, M. I. Stockman, and D. J. Bergman, Self-Similar Chain of Metal Nanospheres as an Efficient Nanolens, Phys. Rev. Lett. 91, 227402 (2003). 
[4] F. Wang and Y. R. Shen, General Properties of Local Plasmons in Metal Nanostructures, Phys. Rev. Lett. 97, 206806 (2006).

[5] V. Klimov, Nanoplasmonics (CRC Press, Boca Raton, FL, 2014).

[6] R. Richtmyer, Dielectric resonators, J. Appl. Phys. 10, 391 (1939).

[7] D. Kajfez and P. Guillon, Dielectric Resonators (Noble, Atlanta, 1998).

[8] S. Long, M. McAllister, and L. Shen, The resonant cylindrical dielectric cavity antenna, IEEE Trans. Antennas Propag. 31, 406 (1983).

[9] R. K. Mongia and P. Bhartia, Dielectric resonator antennas: A review and general design relations for resonant frequency and bandwidth, Int. J. Microw. Millim.-Wave Comput.-Aided Eng. 4, 230 (1994).

[10] A. García-Etxarri, R. Gómez-Medina, L. S. Froufe-Pérez, C. López, L. Chantada, F. Scheffold, J. Aizpurua, M. NietoVesperinas, and J. J. Sáenz, Strong magnetic response of submicron silicon particles in the infrared, Opt. Express 19, 4815 (2011).

[11] A. B. Evlyukhin, S. M. Novikov, U. Zywietz, R. L. Eriksen, C. Reinhardt, S. I. Bozhevolnyi, and B. N. Chichkov, Demonstration of magnetic dipole resonances of dielectric nanospheres in the visible region, Nano Lett. 12, 3749 (2012).

[12] A. I. Kuznetsov, A. E. Miroshnichenko, Y. H. Fu, J. Zhang, and B. Luk'Yanchuk, Magnetic light, Sci. Rep. 2, 492 (2012).

[13] A. I. Kuznetsov, A. E. Miroshnichenko, M. L. Brongersma, Y. S. Kivshar, and B. Luk'yanchuk, Optically resonant dielectric nanostructures, Science 354, aag2472 (2016).

[14] M. V. Rybin, K. L. Koshelev, Z. F. Sadrieva, K. B. Samusev, A. A. Bogdanov, M. F. Limonov, and Y. S. Kivshar, High- $q$ Supercavity Modes in Subwavelength Dielectric Resonators, Phys. Rev. Lett. 119, 243901 (2017).

[15] C. Forestiere, G. Miano, G. Rubinacci, M. Pascale, A. Tamburrino, R. Tricarico, and S. Ventre, Magnetoquasistatic resonances of small dielectric objects, Phys. Rev. Res. 2, 013158 (2020).

[16] J. Van Bladel, On the resonances of a dielectric resonator of very high permittivity, IEEE Trans. Microwave Theory Tech. 23, 199 (1975).

[17] E. A. Muljarov, W. Langbein, and R. Zimmermann, BrillouinWigner perturbation theory in open electromagnetic systems, Europhys. Lett. 92, 50010 (2011).

[18] P. Lalanne, W. Yan, K. Vynck, C. Sauvan, and J.-P. Hugonin, Light interaction with photonic and plasmonic resonances, Laser Photon. Rev. 12, 1700113 (2018).

[19] R. Garbacz and R. Turpin, A generalized expansion for radiated and scattered fields, IEEE Trans. Antennas Propag. 19, 348 (1971).

[20] R. Harrington, J. Mautz, and Y. Chang, Characteristic modes for dielectric and magnetic bodies, IEEE Trans. Antennas Propag. 20, 194 (1972).

[21] D. J. Bergman and D. Stroud, Theory of resonances in the electromagnetic scattering by macroscopic bodies, Phys. Rev. B 22, 3527 (1980).

[22] C. Forestiere and G. Miano, Material-independent modes for electromagnetic scattering, Phys. Rev. B 94, 201406(R) (2016).

[23] X. Zheng, V. Volskiy, V. K. Valev, G. A. E. Vandenbosch, and V. V. Moshchalkov, Line position and quality factor of plasmonic resonances beyond the quasi-static limit: A full-wave eigenmode analysis route, IEEE J. Sel. Top. Quantum Electron. 19, 4600908 (2013).

[24] J. R. de Lasson, J. Mørk, and P. T. Kristensen, Threedimensional integral equation approach to light scattering, extinction cross sections, local density of states, and quasinormal modes, J. Opt. Soc. Am. B 30, 1996 (2013).

[25] C. Forestiere, G. Miano, G. Rubinacci, A. Tamburrino, R. Tricarico, and S. Ventre, Volume integral formulation for the calculation of material independent modes of dielectric scatterers, IEEE Trans. Antennas Propag. 66, 2505 (2018).

[26] J. Mäkitalo, M. Kauranen, and S. Suuriniemi, Modes and resonances of plasmonic scatterers, Phys. Rev. B 89, 165429 (2014).

[27] D. A. Powell, Resonant dynamics of arbitrarily shaped metaatoms, Phys. Rev. B 90, 075108 (2014).

[28] G. D. Bernasconi, J. Butet, and O. J. F. Martin, Mode analysis of second-harmonic generation in plasmonic nanostructures, J. Opt. Soc. Am. B 33, 768 (2016).

[29] D. A. Powell, Interference between the Modes of an AllDielectric Meta-Atom, Phys. Rev. Appl. 7, 034006 (2017).

[30] C. Forestiere, G. Gravina, G. Miano, M. Pascale, and R. Tricarico, Electromagnetic modes and resonances of twodimensional bodies, Phys. Rev. B 99, 155423 (2019).

[31] C. Forestiere, G. Miano, M. Pascale, and R. Tricarico, Electromagnetic scattering resonances of quasi-1-d nanoribbons, IEEE Trans. Antennas Propag. 67, 5497 (2019).

[32] Q. Bai, M. Perrin, C. Sauvan, J.-P. Hugonin, and P. Lalanne, Efficient and intuitive method for the analysis of light scattering by a resonant nanostructure, Opt. Express 21, 27371 (2013).

[33] T. Wu, A. Baron, P. Lalanne, and K. Vynck, Intrinsic multipolar contents of nanoresonators for tailored scattering, Phys. Rev. A 101, 011803(R) (2020).

[34] C. Forestiere and G. Miano, On the nanoparticle resonances in the full-retarded regime, J. Opt. 19, 075601 (2017).

[35] M. Pascale, G. Miano, R. Tricarico, and C. Forestiere, Fullwave electromagnetic modes and hybridization in nanoparticle dimers, Sci. Rep. 9, 14524 (2019).

[36] K. N. Pichugin and A. F. Sadreev, Interaction between coaxial dielectric disks enhances the Q factor, J. Appl. Phys. 126, 093105 (2019).

[37] X. Zambrana-Puyalto and N. Bonod, Purcell factor of spherical Mie resonators, Phys. Rev. B 91, 195422 (2015).

[38] A. Krasnok, S. Glybovski, M. Petrov, S. Makarov, R. Savelev, P. Belov, C. Simovski, and Y. Kivshar, Demonstration of the enhanced Purcell factor in all-dielectric structures, Appl. Phys. Lett. 108, 211105 (2016).

[39] R. Regmi, J. Berthelot, P. M. Winkler, M. Mivelle, J. Proust, F. Bedu, I. Ozerov, T. Begou, J. Lumeau, H. Rigneault et al., Alldielectric silicon nanogap antennas to enhance the fluorescence of single molecules, Nano Lett. 16, 5143 (2016).

[40] S. Mignuzzi, S. Vezzoli, S. A. R. Horsley, W. L. Barnes, S. A. Maier, and R. Sapienza, Nanoscale design of the local density of optical states, Nano Lett. 19, 1613 (2019).

[41] P. R. Wiecha, A. Arbouet, C. Girard, A. Lecestre, G. Larrieu, and V. Paillard, Evolutionary multi-objective optimization of colour pixels based on dielectric nanoantennas, Nat. Nanotech. 12, 163 (2017).

[42] N. Bonod, S. Bidault, G. W. Burr, and M. Mivelle, Evolutionary optimization of all-dielectric magnetic nanoantennas, Adv. Opt. Mater. 7, 1900121 (2019). 
[43] R. F. Harrington, Effect of antenna size on gain, bandwidth, and efficiency, J. Res. Nat. Bur. Stand. 64D, 1 (1960).

[44] R. Collin and S. Rothschild, Evaluation of antenna Q, IEEE Trans. Antennas Propag. 12, 23 (1964).

[45] R. C. Hansen, Fundamental limitations in antennas, Proc. IEEE 69, 170 (1981).

[46] J. S. McLean, A re-examination of the fundamental limits on the radiation $\mathrm{Q}$ of electrically small antennas, IEEE Trans. Antennas Propag. 44, 672 (1996).

[47] A. D. Yaghjian and S. R. Best, Impedance, bandwidth, and Q of antennas, IEEE Trans. Antennas Propag. 53, 1298 (2005).

[48] M. Gustafsson, C. Sohl, and G. Kristensson, Physical limitations on antennas of arbitrary shape, Proc. R. Soc. London, Ser. A 463, 2589 (2007).

[49] H. A. Wheeler, Fundamental limitations of small antennas, Proc. IREE 35, 1479 (1947).

[50] L. J. Chu, Physical limitations of omni-directional antennas, J. Appl. Phys. 19, 1163 (1948).

[51] I. D. Mayergoyz, D. R. Fredkin, and Z. Zhang, Electrostatic (plasmon) resonances in nanoparticles, Phys. Rev. B 72, 155412 (2005).

[52] M. I. Tribelsky and B. S. Luk'yanchuk, Anomalous Light Scattering by Small Particles, Phys. Rev. Lett. 97, 263902 (2006).

[53] R. De Smedt, Correction due to a finite permittivity for a ring resonator in free space, IEEE Trans. Microwave Theory Tech. 32, 1288 (1984).

[54] N. Engheta, Circuits with light at nanoscales: Optical nanocircuits inspired by metamaterials, Science 317, 1698 (2007).

[55] I. D. Mayergoyz, Plasmon Resonances in Nanoparticles (World Scientific, Singapore, 2013), Vol. 6.

[56] J.-M. Jin, Theory and Computation of Electromagnetic Fields (John Wiley \& Sons, New York, 2011).

[57] J. G. Van Bladel, Electromagnetic Fields, (John Wiley \& Sons, New York, 2007), Vol. 19.

[58] G. W. Hanson and A. B. Yakovlev, Operator Theory for Electromagnetics: An Introduction (Springer Science \& Business Media, Berlin, 2013).

[59] C. Forestiere, G. Miano, M. Pascale, and R. Tricarico, Directional scattering cancellation for an electrically large dielectric sphere, Opt. Lett. 44, 1972 (2019).

[60] G. Rosolen, B. Maes, P. Y. Chen, and Y. Sivan, Overcoming the bottleneck for quantum computations of complex nanophotonic structures: Purcell and Förster resonant energy transfer calculations using a rigorous mode-hybridization method, Phys. Rev. B 101, 155401 (2020).
[61] S. G. Mikhlin, Mathematical Physics, an Advanced Course (North-Holland, Amsterdam, 1970), Vol. 11.

[62] L. V. Kantorovich and G. P. Akilov, Functional Analysis (Pergamon, Oxford, UK, 1982).

[63] U. Kreibig and M. Vollmer, Optical Properties of Metal Clusters (Springer Science \& Business Media, Berlin, 2013), Vol. 25.

[64] S. A. Maier, Plasmonics: Fundamentals and Applications (Springer Science \& Business Media, Berlin, 2007).

[65] K. S. Johnson, Transmission Circuits for Telephonic Communication (D. Van Nostrand, New York, 1939).

[66] R. Graglia, Static and dynamic potential integrals for linearly varying source distributions in two- and three-dimensional problems, IEEE Trans. Antennas Propag. 35, 662 (1987).

[67] R. D. Graglia, On the numerical integration of the linear shape functions times the 3-d Green's function or its gradient on a plane triangle, IEEE Trans. Antennas Propag. 41, 1448 (1993).

[68] C. F. Bohren and D. R. Huffman, Absorption and Scattering of Light by Small Particles (John Wiley \& Sons, New York, 2008).

[69] A. Doicu, T. Wriedt, and Y. A. Eremin, Light Scattering by Systems of Particles (Springer-Verlag, Berlin, 2006).

[70] M. I. Mishchenko, L. D. Travis, and A. A. Lacis, Scattering, Absorption, and Emission of Light by Small Particles (Cambridge University Press, Cambridge, UK, 2002).

[71] E. I. Green, The story of Q, Am. Sci. 43, 584 (1955).

[72] S. A. Maier, P. G. Kik, and H. A. Atwater, Optical pulse propagation in metal nanoparticle chain waveguides, Phys. Rev. B 67, 205402 (2003).

[73] G. Colas des Francs, S. Derom, R. Vincent, A. Bouhelier, and A. Dereux, Mie plasmons: Modes, volumes, quality factors, and coupling strengths (Purcell factor) to a dipolar emitter, Int. J. Opt. 2012, 175162 (2012).

[74] R. F. Harrington, Field Computation by Moment Methods (Wiley-IEEE Press, New York, 1993).

[75] J. D. Jackson, Classical Electrodynamics (Wiley, New York, 1998).

[76] D. C. Tzarouchis, P. Ylä-Oijala, and A. Sihvola, Unveiling the scattering behavior of small spheres, Phys. Rev. B 94, 140301(R) (2016).

[77] D. Tzarouchis and A. Sihvola, Light scattering by a dielectric sphere: Perspectives on the Mie resonances, Appl. Sci. 8, 184 (2018).

Correction: The title contained a misspelled word and has been fixed. 